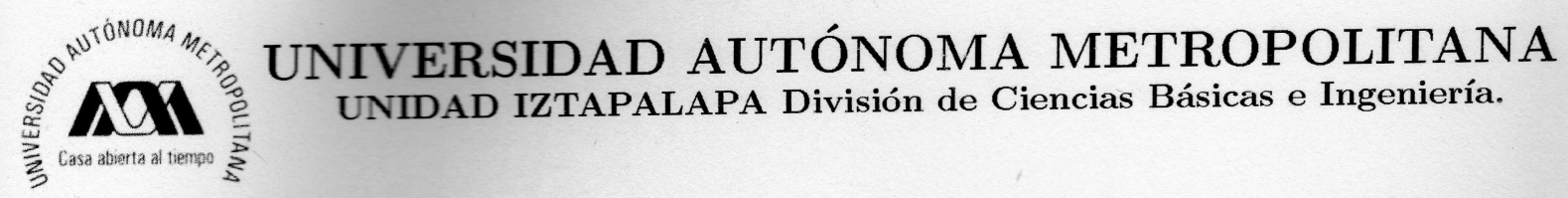

\title{
Análisis de mecanismos de calidad de servicio para aplicaciones multimedia en IEEE 802.11e
}

Idónea Comunicación de Resultados presentada por

\section{Emilio Rafael Olvera Ochoa}

Para obtener el grado de:

Maestro en Ciencias y

Tecnologías de la Información

En el área : Redes y Telecomunicaciones

del Departamento de Ingeniería Eléctrica
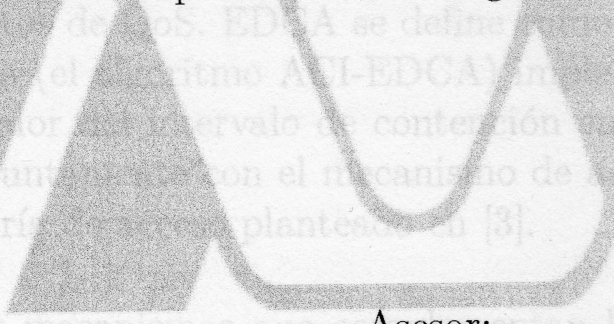

\section{Asesor:}

Dr. Víctor Manuel Ramos Ramos.

Defendida públicamente en la UAM-Iztapalapa el 29 de mayo de 2012 a las 09:00 hrs frente al jurado integrado por :

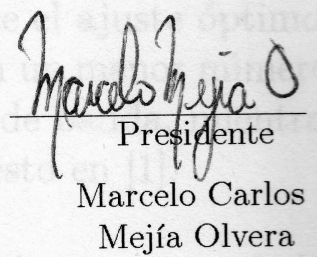

ITAM

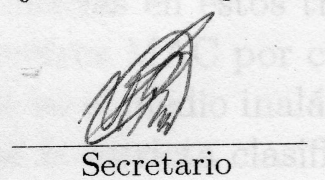

Víctor Manuel

Ramos Ramos

UAM-I

Redes y Telecomunicaciones

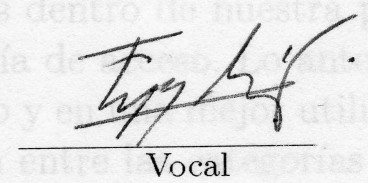

Enrique Rodríguez de la Colina

UAM-I

Redes y Telecomunicaciones 


\section{Resumen}

Este trabajo está enfocado en el análisis y mejora de las funciones de acceso al medio inalámbrico en redes de área local inalámbrica (wireless local area network o WLAN) para soportar la calidad de servicio (QoS) requerida por los servicios multimedia y de comunicación, como pueden ser la telefonía IP, videoconferencia en línea o el vídeo a la demanda (VoD). Con este fin, proponemos una mejora sobre el estándar IEEE 802.11e [1] el cual integra el soporte de calidad de servicio a las redes WLAN existentes (IEEE 802.11a/b/g).

Durante su desarrollo, hemos depurado el algoritmo de gestión de flujos de paquetes basado en prioridad utilizado por EDCA, EDCA es un esquema de acceso al medio descentralizado que permite asignar prioridades entre los flujos de tráfico al interior de la estación en base a sus requisitos de QoS. EDCA se define como parte del estándar IEEE 802.11e [1]. En nuestra propuesta (el algoritmo ACI-EDCA) implementamos el mecanismo dinámico de asignación para el valor del intervalo de contención en base al factor de pérdida propuesto en [2] operando conjuntamente con el mecanismo de asignación de prioridades basado en la actividad por categoría de acceso planteado en [3].

Debido a que los mecanismos que complementan las técnicas adaptativas en cada uno de los algoritmos no mostraban relación con el estado actual de la red o con las condiciones de competencia entre los flujos de tráfico dentro de una misma categoría de acceso, la propuesta de modificación sobre el algoritmo EDCA en [2] y [3] no es capaz de adaptarse de forma óptima al comportamiento real del tráfico fluyendo através de la red y por lo tanto el valor de los parámetros MAC no refleja el cambio de estado al variar las condiciones del medio.

Si bien, tanto en [2] como en [3] se reportan mejoras sobre el estándar, el resultado de integrar las técnicas adaptables presentadas en estos trabajos dentro de nuestra propuesta, permite el ajuste óptimo en los parámetros MAC por categoría de acceso. Lo anterior se refleja en un menor número de colisiones en el medio inalámbrico y en una mejor utilización del ancho de banda, mientras se mantiene la estricta clasificación entre las categorías de acceso propuesto en [1].

Implementamos el algoritmo ACI-EDCA y evaluamos su desempeño mediante simulación utilizando NS-2 [4], que es de licencia gratuita y de código abierto, ampliamente utilizado por la comunidad científica. 


\section{Abstract}

This work is focused to the analysis and improvement of the medium access function to provide the required QoS for the services of multimedia and communication, as can be IP telephony, online videoconference or the video on demand (VoD). With this purpose, we propose an improvement over the standard IEEE 802.11e [1] that integrates the QoS support to the existent WLAN networks (IEEE $802.11 \mathrm{a} / \mathrm{b} / \mathrm{g}$ ).

During their development, we depurate the algorithm to manage the packet flows based in priority used for EDCA, EDCA is a descentralized medium access scheme wich allows asign priorities between the traffic flows inside the wireless station in basis of their QoS requirements. EDCA is defined as part of the IEEE 802.11e standard [1]. In our proposal (the ACI-EDCA algorithm) we implemented the dynamic asignation mechanism for the value of the contention interval based in the access category activity proposed in [2] operating jointly with the mechanism of priorities assignation based in the activity per access category as propounded in $[3]$.

Because the mechanisms that complement the adaptative techniques in each of the algorithms do not show relation with the current state of the network or the competing conditions between the traffic flows inside the same access category, the proposed modification over the EDCA algorithm in [2] and [3] is not capable to adapt optimally the real behavior of the traffic flowing through the network and therefore the value of the MAC parameters does not reflect the state change when the medium condition varies.

Even though, both [2] as in [3] were reported improvements over the standard, the result of integrate the adaptative techniques presented in this works in our proposal, allows the optimal adjust in the MAC parameters for access category. This are reflected in a less number of collisions in the wireless medium and in a better utilization of the bandwidth, while is maintained the strict classification between the access categories proposed in [1].

We implemented the ACI-EDCA algorithm and evaluate their performance through simulation utilizing N-S2 [4], which is freeware and open source, widely used by the scientific community. 


\section{Agradecimientos}

Agradezco profundamente al Dr. Víctor Manuel Ramos Ramos, mi asesor, por aceptarme en este proyecto y orientarme durante la realización de este trabajo. Por todos sus consejos y extensas correcciones durante el desarrollo del mismo. Sin su sabia guía la culminación de está empresa no hubiese sido posible. También le doy las gracias por formar parte del jurado para la defensa pública de este proyecto.

Extiendo mi agradecimiento al Dr. Enrique Rodríguez de la Colina y al Dr. Marcelo Carlos Mejía Olvera por hacerme el honor de participar en el jurado para la defensa pública de este trabajo. Gracias por todos sus comentarios y sugerencias, los cuales enriquecieron enormemente el trabajo aquí presentado. También agradezco a todos los profesores de la Maestría por sus consejos, sus recomendaciones y sus valiosas enseñanzas.

Mi más profunda gratitud a toda mi familia. Especialmente, a mi madre Ma. Clara, a mi padre J. Guadalupe, a mi hermano Saul y a mi hermana Laura. Que siempre han estado a mi lado y me han apoyado, aún cuando mi estado de ánimo no haya sido siempre el mejor.

A mi novia Jacqueline por su gran apoyo, paciencia, tolerancia, comprensión. Forma parte esencial en mi vida y me siento muy afortunado de tenerla a mi lado.

Extiendo mi gratitud a mis compañeros y amigos del PCyTI, por su apoyo, animos, conocimientos e ideas aportadas.

Vaya mi gratitud a la Universidad Autónoma Metropolitana por darme la oportunidad de desarrollarme académicamente y Al Consejo Nacional de Ciencia y Tecnología por el financiamiento que me otorgó para la realización de mis estudios de maestría.

Finalmente agradezco de manera especial a K. y a C. por su apoyo incodicional. 


\section{Contenido}

$\begin{array}{lll}\text { Lista de Figuras } & \text { XI }\end{array}$

Lista de Tablas $\quad$ XIII

1. Introducción $\quad 1$

2. Fundamentos de IEEE 802.11 3

3. Funciones de acceso al medio en IEEE 802.11 7

3.1. Función de coordinación distribuida $(\mathrm{DCF}) \ldots \ldots \ldots \ldots$

3.2. Función de coordinación puntual $(\mathrm{PCF}) \ldots \ldots \ldots \ldots$

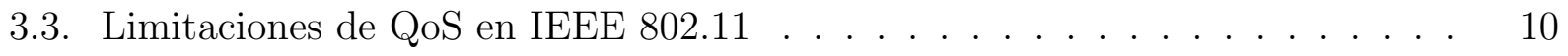

3.3.1. Limitaciones en DCF . . . . . . . . . . . . . . . . . 10

3.3.2. Limitaciones en $\mathrm{PCF} \ldots \ldots \ldots 11$

4. El estándar para QoS: IEEE 802.11e 13

4.1. Función de acceso al canal controlado por HCF (HCCA) . . . . . . . . . . . 14

4.2. Acceso coordinado y distribuido mejorado (EDCA) . . . . . . . . . . 15

4.3. Limitaciones de QoS en IEEE 802.11e . . . . . . . . . . . . . . . . . . . . . . . . . . . . . . .

4.3.1. Limitaciones en HCCA. . . . . . . . . . . . . . . . . . . . . . . . . . . . . . . .

4.3.2. Limitaciones en EDCA. . . . . . . . . . . . . . . . . . 17

5. Estado del arte $\quad 19$

5.1. Algoritmo RAMPS-EDCA . . . . . . . . . . . . . . . . . . . . 20

5.1.1. Etapa de selección de la ventana de contención . . . . . . . . . . . 20

5.1.2. Etapa de selección del valor AIFS . . . . . . . . . . . . . . . 22

5.2. Algoritmo CI-EDCA . . . . . . . . . . . . . . . . . . . . . . . . . . . . . . . . . . .

5.2.1. Esquema modificado de acceso EDCA para QoS . . . . . . . . . 23

5.2.2. Esquema mejorado para la asignación de prioridad basado en CW . . 25

6. Algoritmo ACI-EDCA 29

6.1. Descripción de la propuesta . . . . . . . . . . . . . . . . . 29 
7. El modelo EDCA: metodología de evaluación 35

7.1. Consideraciones para el modelo de tráfico en EDCA . . . . . . . . . . . . . 37

7.2. Métricas de desempeño para evaluar la calidad de servicio . . . . . . . . . 38

8. Simulaciones y resultados $\quad 41$

8.1. Fuentes de tráfico . . . . . . . . . . . . . . . . . . . . 41

8.2. Parámetros de simulación . . . . . . . . . . . . . . . . 43

8.3. Resultados . . . . . . . . . . . . . . . . . . . . . . 44

9. Conclusiones y perspectivas $\quad 49$

$\begin{array}{ll}\text { Referencias } & 51\end{array}$

A. El simulador NS-2 $\quad 55$

A.1. Características generales . . . . . . . . . . . . . . 55

A.1.1. WLAN en NS-2 . . . . . . . . . . . . . . . . 56

A.1.2. IEEE 802.11e en NS-2 . . . . . . . . . . . . . . 57

$\begin{array}{ll}\text { Acrónimos } & 61\end{array}$ 


\section{Lista de Figuras}

2.1. Pila de protocolos para el estándar IEEE $802.11 \ldots \ldots$. . . . . . . . . . 3

2.2. Canales en IEEE 802.11b. . . . . . . . . . . . . . . . . . . . . . . . 4

3.1. Método de acceso básico en DCF. . . . . . . . . . . . . . . . 7

3.2. Esquema de acceso mediante PCF (ciclo PCF+DCF). . . . . . . . . . . . . 9

3.3. Imposición de zonas de silencio mediante RTS-CTS. . . . . . . . . . . . . . . . . 10

4.1. Acceso al medio mediante EDCA. . . . . . . . . . . . . . . . . . . . . . 13

4.2. Funciones de acceso al medio en el estándar IEEE 802.11e. . . . . . . . . . . . . 14

4.3. Acceso al medio mediante HCCA. . . . . . . . . . . . . . . . . . . . . . . . 14

4.4. Esquema de la función de acceso al medio EDCA. . . . . . . . . . . . . . . 16

5.1. Diagrama de la estructura propuesta para el acceso al canal mediante EDCA. . . 21

5.2. Relación inter-trama modificada para EDCA . . . . . . . . . . . . . . 24

5.3. Nuevo esquema de clasificación inter-nodo basado en la actividad de la estación para EDCA. . . . . . . . . . . . . . . . 26

6.1. Esquema activo de clasificación inter-estación para EDCA. . . . . . . . . . . . . 29

6.2. Lógica de acceso al medio propuesta. . . . . . . . . . . . . . . . . . . . . 31

7.1. Proceso de arribo de paquetes a la capa MAC. . . . . . . . . . . . . . . 36

7.2. Cadena de Markov para la fuente ON/OFF . . . . . . . . . . . . . . 37

7.3. Patrón de tráfico generado a partir de la fuente $\mathrm{ON} / \mathrm{OFF}$ exponencial. . . . . . . 38

8.1. Topología de la red a simular. . . . . . . . . . . . . . . . . . . 43

8.2. Colisiones virtuales detectadas durante la asignación del turno de transmisión. . . 44

8.3. Paquetes en colisión durante su transmisión por el canal inalámbrico. . . . . . . . 44

8.4. Comparación de desempeño para el throughput por AC. . . . . . . . . . . . . . 45

8.5. Comparación de desempeño para el retardo por AC . . . . . . . . . . . . . . 46

8.6. Comparación de desempeño para la varianza por AC . . . . . . . . . . . . . . . 47

A.1. Dualidad entre clases. . . . . . . . . . . . . . . . . . . . 56

A.2. Esquema simplificado de los elementos de NS-2 . . . . . . . . . . . . . 57

A.3. Estructura de jerarquías para la clase MobileNode en NS-2 . . . . . . . . . . . 58

A.4. Estructura de jerarquías propuesta en [5] para la clase MobileNode de NS-2. . . . 59 


\section{Lista de Tablas}

2.1. Requerimientos por aplicación sobre los recursos del sistema . . . . . . . . . . . . 5

5.1. Rango de desplazamiento para los valores de AIFSN por AC. . . . . . . . . . . . 23

5.2. Parámetros EDCA usados en la implementación de la propuesta. . . . . . . . . . 25

5.3. Comparación entre las propuestas para QoS en IEEE 802.11 . . . . . . . . . . . 27

8.1. Parámetros de simulación para EDCA. . . . . . . . . . . . . . . . . . 42

8.2. Parámetros de la capa MAC y de la capa física. . . . . . . . . . . . . . . . . . . 44 


\section{Capítulo 1 Introducción}

En los últimos años, el despliegue de las tecnologías inalámbricas en el sector de las telecomunicaciones ha experimentado un crecimiento exponencial. La evolución actual de la sociedad hacia la sociedad de la información ha contribuido de forma decisiva a este auge. Entre las nuevas tecnologías de comunicación masivas desplegadas, sobresalen las comunicaciones mediante teléfonos móviles, computadoras portátiles, entre otros dispositivos que por la naturaleza de su entorno ubicuo, la comunicación por el medio inalámbrico resulte indispensable.

Aunado a lo anterior, se ha producido un notable aumento en la demanda del número de aplicaciones multimedia sobre medios inalámbricos, tales como voz sobre IP (VoIP), audio conferencia, video conferencia, audio y video a la demanda (AVoD). Estas aplicaciones requieren que los servicios suministrados a los usuarios ofrezcan garantías de calidad de servicio (QoS) en cuanto al ancho de banda, retardo de extremo a extremo (latencia), variabilidad en el retardo (jitter) o tasa de error. En concreto, muchas aplicaciones multimedia son sensibles a la variabilidad en la tasa de transmisión, al retardo y a la variabilidad en el retardo, aunque toleran cierta pérdida de datos. Por otro lado, tenemos a las aplicaciones clásicas, flexibles al retardo y a la disponibilidad de ancho de banda, como pueden ser el correo electrónico, la transferencia de archivos entre equipos remotos, la navegación web y el login remoto, que en general tienen requisitos distintos a las aplicaciones multimedia. En estas aplicaciones, la variabilidad en el retardo o en la disponibilidad de ancho de banda, aunque actúan en su detrimento, no impiden que se realice el servicio.

Tanto las aplicaciones multimedia como las clásicas utilizan IP como protocolo a nivel de la capa de red. Este protocolo proporciona un servicio de mejor esfuerzo (best-effort) para todos los datos enviados, sin diferenciar el tipo de tráfico o sus requisitos de QoS, es decir, ofrece un servicio de interconexión de redes independiente de los requisitos del usuario, con un tratamiento equitativo para todos los datagramas IP.

Es en este escenario que IEEE 802.11 [6] se ha convertido en el estándar de facto para las redes inalámbricas de área local (WLANs). Este trabajo se centra en el estudio de las redes WLAN para soportar la calidad de servicio requerida por los servicios multimedia típicos de comunicación, telefonía, vídeo a la demanda y servicios básicos de Internet.

Actualmente se cuenta con un estándar que permite mejorar la calidad de servicio en las redes WLAN existentes (802.11a/b/g): el estándar 802.11e [1]. Para cumplir con este 
objetivo, IEEE 802.11e introduce un nuevo elemento denominado función de coordinación híbrida de acceso al medio (HCF). El estándar contempla dos modos de operación en HCF, el modo distribuido basado en contención (EDCA) y el modo centralizado de acceso (HCCA). En EDCA el acceso al canal se concede en base a una estrategia distribuida durante la etapa de contención, mientras que bajo el modo HCCA el acceso al canal sigue un esquema centralizado libre de contención y controlado por el coordinador híbrido (o HC). HCF permite que EDCA opere de forma concurrente con el modo HCCA. EDCA está orientado a servicios con QoS priorizado y HCCA fue diseñado para garantizar servicio de QoS estricto.

Debido a la incertidumbre sobre las condiciones del medio inalámbrico, la capacidad de la red para ofrecer garantías sobre requisitos de QoS estrictos durante los periodos de alta carga de tráfico bajo el esquema de HCCA se reduce. En especial, si se considera el estricto control sobre el acceso al medio debido a la alta demanda de las aplicaciones multimedia sobre los recursos de la red.

Por su parte, EDCA como una evolución del mecanismo de acceso distribuido al medio (DCF) para IEEE 802.11, incluye todos los elementos básicos de DCF como el protocolo de acceso múltiple al medio por detección de portadora con prevención de colisiones (CSMA/CA), el procedimiento de backoff o los espacios inter-trama para la gestión del acceso al canal. Aparte de estos elementos básicos comunes, añade nuevos componentes que permiten introducir calidad de servicio en el sistema, como son el concepto de oportunidad de transmisión (TXOP) o el de espacio inter-trama arbitrario (AIFS). EDCA ofrece servicios de tráfico diferenciado, sin reserva de recursos. Por tanto, la asignación de la oportunidad de transmisión se toma basada en decisión por consenso, es decir, la decisión para asignar la TXOP se toma conjuntamente entre todas las estaciones.

El objetivo de este trabajo es la implementación de un algoritmo mejorado de acceso al medio basado en EDCA, que satisfaga las demandas de QoS requeridas por las aplicaciones en redes IEEE 802.11. La implementación se lleva a cabo en NS-2, simulador de licencia gratuita y de código abierto, ampliamente utilizado por la comunidad científica. Posteriormente, se evalúa su rendimiento con diferentes patrones de tráfico para demostrar que se optimiza el uso del ancho de banda compartido. Especialmente, en escenarios con alta demanda de tráfico multimedia de alta prioridad.

Este documento está estructurado de la siguiente manera, en el Capítulo 2 abordamos los conceptos necesarios para comprender el funcionamiento del estándar IEEE 802.11. El Capítulo 3 describe las principales características de las funciones de acceso al medio en IEEE 802.11. En el Capítulo 4 se revisa la especificación IEEE 802.11e, estándar orientado a características de QoS y soporte multimedia. En el Capítulo 5 presentamos el trabajo relacionado, particularmente los algoritmos RAMPS-EDCA [2] y EDCA-CI [3]. En el Capítulo 6 se presentan las consideraciones para el modelo de tráfico para EDCA y las métricas de desempeño a evaluar.El Capítulo 7 describe nuestra propuesta de mejora para el algoritmo EDCA. El Capítulo 8 abarca los resultados obtenidos en la comparativa de desempeño entre el trabajo de referencia y nuestra propuesta. Finalmente, en el Capítulo 9 concluimos discutiendo los resultados obtenidos, las implicaciones de estos y el trabajo a futuro. 


\section{Capítulo 2 Fundamentos de IEEE 802.11}

El estándar IEEE 802.11 [6] cubre la subcapa MAC y la capa física (PHY) en la pila de protocolos TCP/IP del modelo de referencia para arquitecturas de red. La subcapa MAC es la encargada del control de acceso al medio, que determina cómo el medio será usado. La capa física por su parte, especifica el esquema de modulación empleado así como las características de señalización para la transmisión a través de radio frecuencia. En la Figura 2.1 [7] se muestra la arquitectura de la pila de protocolos para el estándar IEEE 802.11. La arquitectura propuesta ofrece una interfaz transparente a los usuarios de las capas superiores: las estaciones (STAs) pueden moverse a través de la WLAN 802.11 y aparentan estar estacionarias para la subcapa de control de enlace lógico (LLC del estándar 802.2) y otras capas superiores. Esto permite que los protocolos TCP/IP existentes funcionen sobre las WLAN IEEE 802.11 de modo similar a como operan en medios cableados bajo el estándar Ethernet.

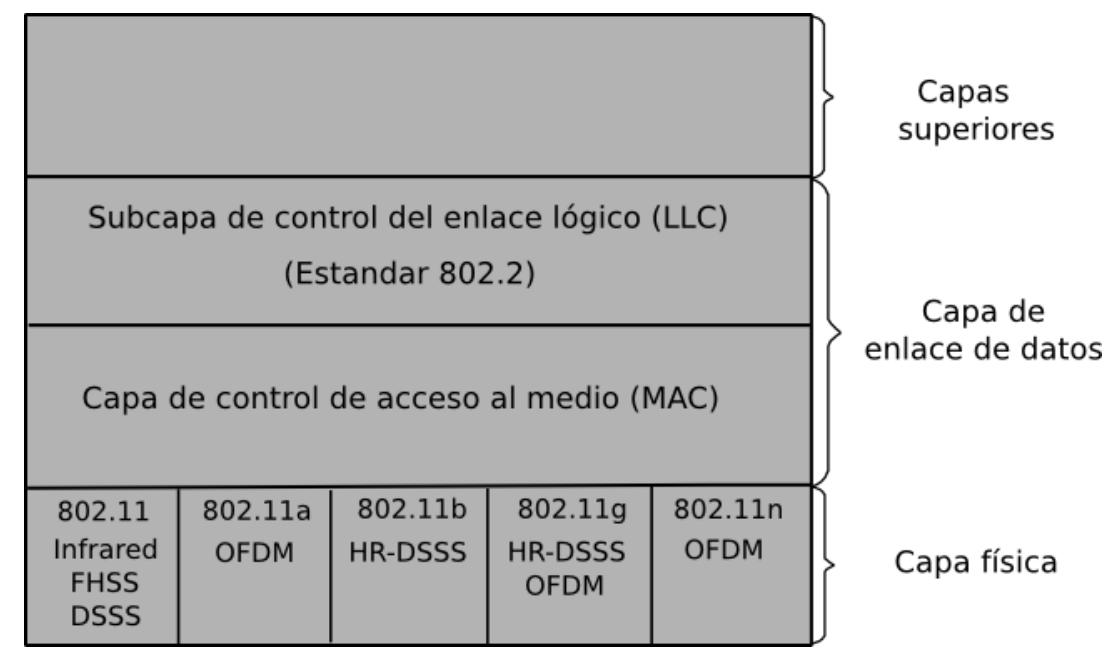

Figura 2.1: Pila de protocolos para el estándar IEEE 802.11

El estándar contempla dos modos de operación: el modo infraestructura y el modo ad-hoc. En modo infraestructura, el bloque de construcción fundamental de la arquitectura de red es el conjunto básico de servicios (o BSS por sus siglas en inglés). Un BSS contiene una o más estaciones inalámbricas y una estación base central, conocida como punto de acceso (AP). 
A través de la conexión entre el AP y un dispositivo de interconexión (como puede ser un concentrador, switch o dispositivo de encaminamiento) el BSS accede a Internet.

En el modo ad-hoc, la red se forma "al vuelo" entre los dispositivos móviles que buscan intercambiar información en ausencia de un AP centralizado. En una red ad-hoc, las estaciones se encuentran dentro de un BSS independiente (IBSS) y se comunican directamente unas con otras. Típicamente, las IBSS's están compuestas por un pequeño número de estaciones preparadas para un propósito específico con conectividad durante un periodo corto de tiempo.

Cada estación IEEE 802.11 tiene una dirección MAC de 6 bytes perteneciente al adaptador de red inalámbrica. Como en el caso de Ethernet, estas direcciones MAC son administradas por la IEEE y (en teoría) deben identificar unívocamente el dispositivo a nivel mundial. Cuando un administrador de red instala un AP, asigna una o dos palabras como identificador del conjunto básico de servicios (SSID) al que pertenece. Adicionalmente, el administrador también deberá asignar un número de canal al AP. El número de canales disponibles depende de la versión del estándar IEEE 802.11, p.ej., para 802.11b que opera en el rango de frecuencias $2.4 \mathrm{GHz}$ a $2.485 \mathrm{GHz}$ dispone de una banda de transmisión de $85 \mathrm{MHz}$. Dentro de estos 85 $\mathrm{MHz}, 802.11 \mathrm{~b}$ define once canales parcialmente traslapados. Dos canales se consideran no traslapados si y solo si se encuentran separados por cuatro o más canales. En particular, como se muestra en la Figura 2.2 [8], los canales uno, seis y once conforman el mayor conjunto de canales no traslapados. Esto significa, por ejemplo, que un administrador puede crear una red local inalámbrica con una tasa de transmisión agregada (la suma de las tasa de transmisión de todas las terminales en la red) de 33 Mbps instalando tres AP's 802.11b con la misma cobertura, asignando los canales uno, seis y once a los AP's e interconectando los AP's mediante un switch.

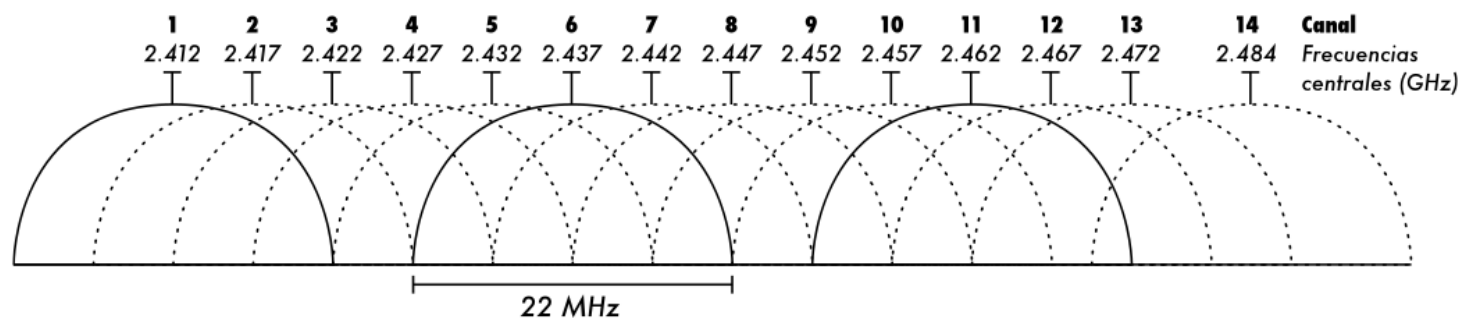

Figura 2.2: Canales en IEEE 802.11b.

Inicialmente no fue necesario ofrecer QoS a los servicios en Internet. Dado que sólo se tenía un tráfico limitado y el intercambio de datos era el predominante. Es por ello que el estándar IEEE 802.11 fue originalmente propuesto para ofrecer exclusivamente servicio de entrega de mejor esfuerzo. En la Tabla 2.1 se observa que, actualmente los diferentes servicios de usuario final (aplicaciones), presentan una demanda diferente de los sistemas de comunicación de los que hacen uso. Es precisamente en este compromiso entre los requerimientos de servicio de la aplicación y lo que la red ofrece donde se tienen a los mecanismos de QoS. 


\begin{tabular}{ccccc}
\hline Aplicación & Confiabilidad & Retardo & Jitter & Ancho de banda \\
\hline E-mail & Alta & Baja & Baja & Baja \\
FTP & Alta & Baja & Baja & Media \\
Acceso a Web & Alta & Media & Baja & Media \\
Login Remoto & Alta & Media & Media & Baja \\
Audio a la demanda & Baja & Baja & Alta & Media \\
Video a la demanda & Baja & Baja & Alta & Alta \\
Telefonía IP & Baja & Alta & Alta & Baja \\
Video Conferencia & Baja & Alta & Alta & Alta \\
\hline
\end{tabular}

Tabla 2.1: Requerimientos por aplicación sobre los recursos del sistema [9]. 


\section{Capítulo 3}

\section{Funciones de acceso al medio en IEEE 802.11}

La subcapa MAC IEEE 802.11 define dos funciones de coordinación de acceso al medio: DCF y la función de coordinación puntual (o PCF por sus siglas en inglés), que describimos a continuación.

\subsection{Función de coordinación distribuida (DCF)}

En la Figura 3.1 [10], se muestra el modo de operación de DCF basado en contención y con soporte para transmisión asíncrona. El modo DCF es obligatorio en todas las estaciones 802.11, mientras que el modo PCF es opcional.

DCF usa un mecanismo CSMA/CA para regular el acceso al medio inalámbrico compartido. Si el medio se detecta inactivo por un intervalo de tiempo, denominado DIFS (espacio inter-trama en DCF), la estación entra en un periodo de espera (intervalo de backoff) descrito por:

$$
\text { Intervalo }_{\text {backoff }}=\operatorname{rand}(0, C W) \times \text { tiempo_ranura }
$$

Donde el intervalo de backoff se genera aleatoriamente a partir del tamaño de la ventana de contención $(C W)$. En el primer intento de transmisión, $C W$ es igual a su valor mínimo,

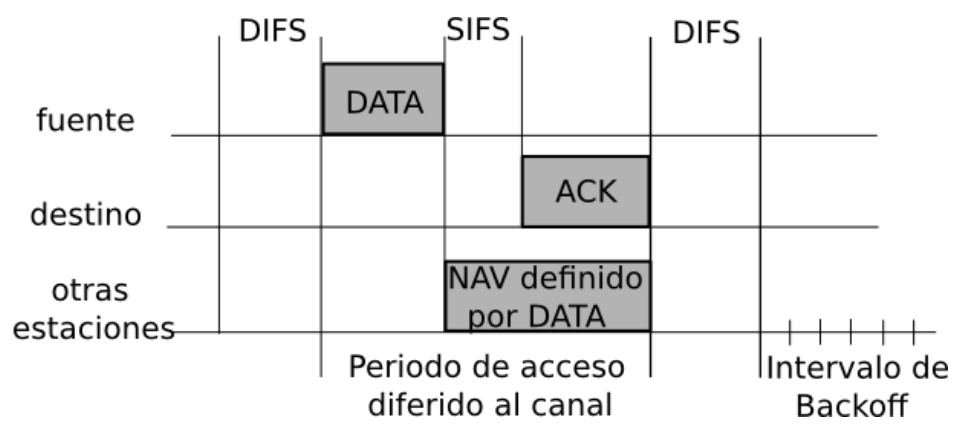

Figura 3.1: Método de acceso básico en DCF. 
CWmin. Su valor se duplica después de cada intento de transmisión no exitoso hasta alcanzar un valor máximo, CWmax. El valor de $C W$ regresa a CWmin después de cada transmisión exitosa. El contador del tiempo de espera se decrementa cada vez que durante la duración total del tiempo de ranura el medio se detecta inactivo. El contador se "congela" si el medio se detecta ocupado, y se reanuda después de que el medio pasa a inactivo nuevamente, por otro intervalo de tiempo DIFS. Sólo cuando el contador del intervalo de backoff llega a cero, se podrá transmitir la trama. Una trama de confirmación (ACK) se envía al emisor para confirmar una transmisión correcta. El intervalo de tiempo entre una trama de datos y su ACK es denominado SIFS (intervalo corto de tiempo). Si el ACK no se recibe dentro del límite de tiempo (condición de time-out), se asumirá que ocurrió una colisión y se retransmitirá la trama, hasta que se alcance el número máximo de intentos. El vector de asignación de red (o NAV) es una trama definida por el temporizador local de cada estación; su función es detener los intentos de transmisión por un intervalo definido en el campo de "duración" de la trama.

\subsection{Función de coordinación puntual (PCF)}

El acceso al medio en el modo PCF sigue un esquema libre de contención y con soporte para transmisión síncrona. La regla de acceso al medio en el modo PCF se basan en una estrategia de asignación de turnos centralizado. El mecanismo de elección puede ser un planificador round-robin o bien un esquema basado en prioridades. Como se muestra en la Figura 3.2 [11], en PCF el canal inalámbrico se trata como la estructura de una super-trama (o intervalo de repetición libre de contención), que consiste en un periodo libre de contención (CFP) y un periodo de contención (CP). Al inicio de cada periodo CFP, el punto de acceso inalámbrico (AP) emite una trama de señalización a todas las estaciones dentro del área de servicio (BSA) después de que el AP confirma que el medio se encuentra inactivo por la duración total de un intervalo de tiempo inter-trama (PIFS). PIFS debe ser menor a un intervalo DIFS y no mayor al intervalo SIFS. El estándar define la duración de cada uno de los intervalos de tiempo dependiendo de las características de la capa física.

\section{Espacios entre trama para diferir el acceso al canal en IEEE802.11 [6]}

- Espacio inter-trama SIFS:tiempo de espera antes de realizar transmisiones de alta prioridad (p. ej. tramas de confirmación). También se usa para transmisiones de las estaciones durante el período libre de contención (en el mecanismo de acceso al medio $\mathrm{PCF}$ ). Al ser el menor de los intervalos esperados, no son susceptibles de tener colisiones con otras transmisiones.

- Espacio inter-trama PIFS:tiempo de espera antes de poder transmitir una trama durante el período libre de contención. Como el intervalo de espera en PCF es inferior al de DCF, estas transmisiones tendrán prioridad durante dicho período.

$$
\text { PIFS }=\text { SIFS }+ \text { SlotTime }
$$


- Espacio inter-trama DIFS:tiempo mínimo que el medio debe estar libre antes de poder transmitir en el período de contención.

$$
\text { DIFS }=\text { SIFS }+2 \times \text { SlotTime }
$$

- Espacio entre tramas extendido (EIFS): Espacio usado para la retransmisión de tramas. No tiene un valor fijo, pero su valor es superior al resto del espacio entre tramas.

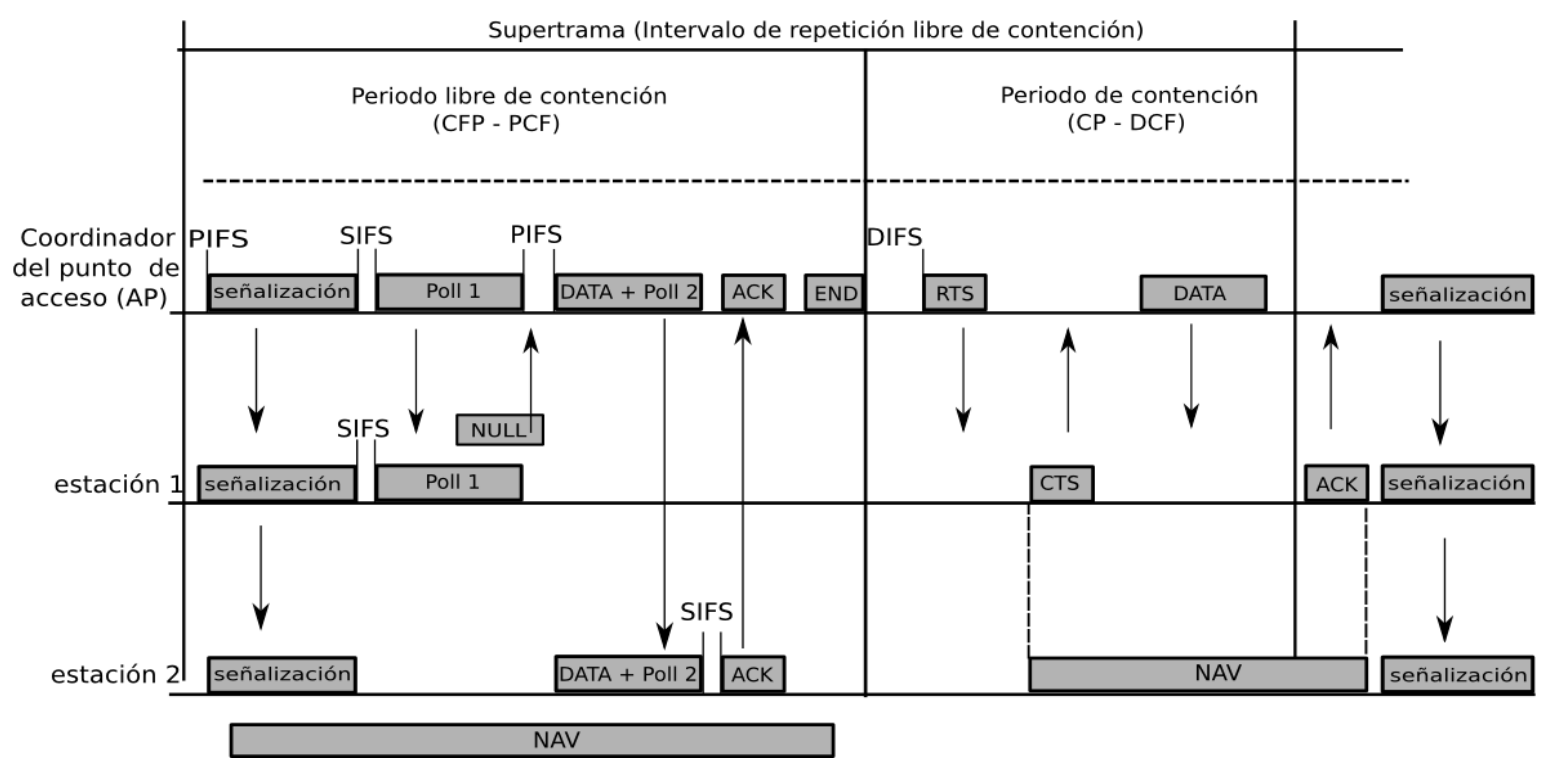

Figura 3.2: Esquema de acceso mediante PCF (ciclo PCF+DCF).

La trama de guía contiene la información sobre la máxima duración del período CFP, la duración del intervalo de guía y el identificador del conjunto básico de servicios (BSS). Todas las estaciones en el BSS ajustan su NAV y no envían ningún paquete en el período CFP después de haber escuchado a la trama de señalización.

Durante el período de CFP el AP detecta cada estación en su lista al enviarle una trama de consenso $D A T A+C F$. Si una estación recibe dicha trama, puede responder al AP después de un intervalo SIFS con una trama $D A T A+C F-A C K$. Si el AP recibe la trama $D A T A+$ $C F-A C K$, puede enviar una trama de consenso $D A T A+C F-A C K+C F$. Una estación que recibe la trama de consenso $D A T A+C F-A C K+C F$ puede responder al AP con una trama DATA o NULL. El AP continúa detectando cada estación hasta alcanzar la máxima duración del período CFP. El AP puede terminar el período CFP al enviar una trama $C F-E N D$. Las tramas RTS y CTS se abordarán con más detalle al revisar los mecanismos que ofrece IEEE 802.11 para tratar el problema de "la terminal escondida". Otras estaciones que escuchen la trama RTS, CTS o de datos (DATA), actualizan el valor de sus temporizadores locales al valor contenido en el campo "duración" y no iniciarán transmisiones hasta que el valor de NAV llegue a cero. 
Además de ofrecer acceso al canal mediante DCF o $P C F+D C F$, la capa MAC de una WLAN IEEE 802.11 necesita ofrecer garantías de QoS y proporcionar seguridad a las aplicaciones de las capas superiores. El ajuste de parámetros de QoS es generalmente un problema que requiere el definir los parámetros de QoS de la aplicación y los parámetros de QoS de la red, con el fin de definir un proceso para las situaciones de negociación donde se gestionan los recursos compartidos dentro de la BSA.

Un problema frecuente en redes Wi-Fi es el de la terminal escondida: algunas estaciones dentro de un mismo BSS no pueden escuchar a otras dentro del conjunto. Con ello, se podría presentar la transmisión simultánea hacia un receptor común. Para este caso, IEEE 802.11 define un esquema opcional denominado algoritmo de acceso de inicio de conexión de cuatro fases, que se conoce como método de acceso RTS/CTS o algoritmo MACAW (Multiple Access with Collission Avoidance for Wireless). El funcionamiento del método se ilustra en la Figura 3.3. Este esquema se puede asociar al método básico de acceso DCF, cuando la trama de datos excede un cierto valor de umbral RTS. De esta forma el rendimiento del sistema puede ser mejorado si se tiene el problema de terminales ocultas. Sin embargo, si el tamaño de las tramas de datos es pequeño, se recomienda deshabilitar esta opción para evitar degradar el desempeño por sobrecarga de tramas de control.

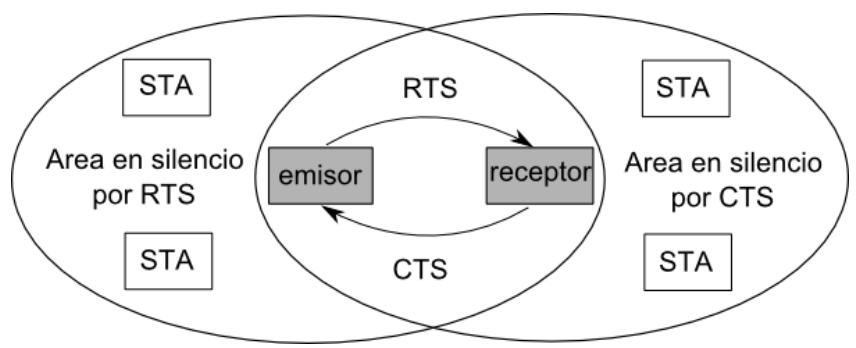

Figura 3.3: Imposición de zonas de silencio mediante RTS-CTS.

\subsection{Limitaciones de QoS en IEEE 802.11}

Existen una serie de inconvenientes en DCF y PCF que impiden garantizar los requisitos mínimos de QoS para las aplicaciones y servicios multimedia, los cuales exponemos a continuación.

\subsubsection{Limitaciones en DCF}

DCF sólo ofrece un servicio de mejor esfuerzo, y no contempla mecanismos de QoS. En DCF las estaciones de un BSS compiten por los recursos y el acceso al canal con el mismo nivel de prioridad. Esta carencia de discriminación de las distintas clases de flujos, puede causar problemas de QoS para las aplicaciones multimedia cuando la carga de tráfico sobre la red es alta. 


\subsubsection{Limitaciones en PCF}

PCF se diseñó para soportar aplicaciones multimedia. Sin embargo, este método de acceso presenta tres problemas fundamentales que llevan al deterioro de QoS:

- PCF define una única clase de algoritmo planificador round-robin, que no puede tratar los diferentes requerimientos de QoS para distintos tipos de tráfico.

- El AP compite por el acceso al medio. Si durante una petición de acceso el medio se encuentra ocupado, la transmisión de la trama de señalización inicial se difiere y se inducen retardos en futuras transmisiones.

- El AP no tiene control sobre la duración de la transmisión de una estación una vez iniciada. Esto impide que el AP ofrezca garantías sobre el retardo o de la variabilidad en el retardo para otras estaciones presentes en su lista de detección durante el resto del intervalo libre de contención (CFP). 
Capítulo 4

\section{El estándar para QoS: IEEE 802.11e}

IEEE 802.11e [1] define a HCF como función de acceso al medio. Una de las principales características de $\mathrm{HCF}$, en el modo EDCA, es la introducción de cuatro registros de almacenamiento para las categorías de acceso (AC), que en su funcionamiento pueden considerarse como ciclos DCF virtuales, (ver Figura 4.1). También, define ocho registros de almacenamiento para diferenciar flujos de tráfico (TS) en HCCA, que son controlados a nivel MAC por el HC.

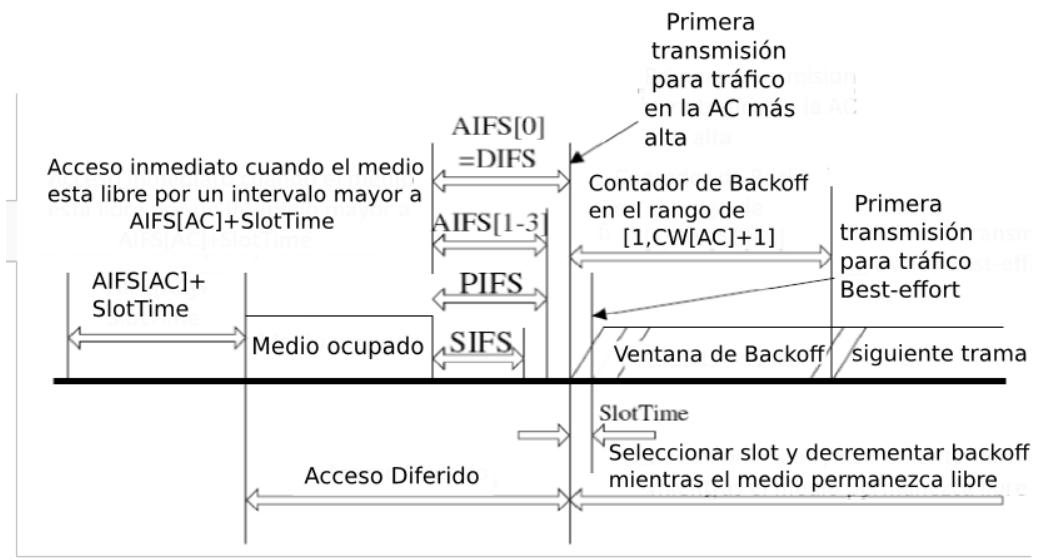

Figura 4.1: Acceso al medio mediante EDCA.

La razón para separar los registros TS de los registros AC, es ofrecer servicio diferenciado para QoS estricto y QoS priorizado respectivamente. QoS estricto es un requerimiento de QoS expresado en forma cuantitativa, como lo es la tasa de transmisión, límite en retardo o límite sobre la variabilidad en el retardo. Se espera que estos valores vengan contenidos en el servicio de datos de la capa MAC como información de especificación de tráfico (TSPEC) durante la transferencia de datos entre pares de estaciones. QoS priorizado se expresa en términos relativos a la prioridad en la entrega; esto se usa con el servicio de datos de la capa MAC en la transferencia de tramas de datos entre pares de estaciones. Los valores de los parámetros de QoS, tales como la tasa de transmisión, el límite en el retardo o el límite en la variabilidad del retardo, pueden variar en la transferencia de las tramas de datos, sin necesidad de reservar la asignación de recursos mediante la negociación de TSPEC entre la 
estación y el AP.

EDCA fue diseñado para soportar tráfico priorizado, mientras que HCCA soporta tráfico parametrizado. Para asegurar la compatibilidad de redes inalámbricas con QoS y sin QoS el estándar [1] permite la existencia conjunta de DCF y PCF con HCF como se puede observar en la Figura 4.2.

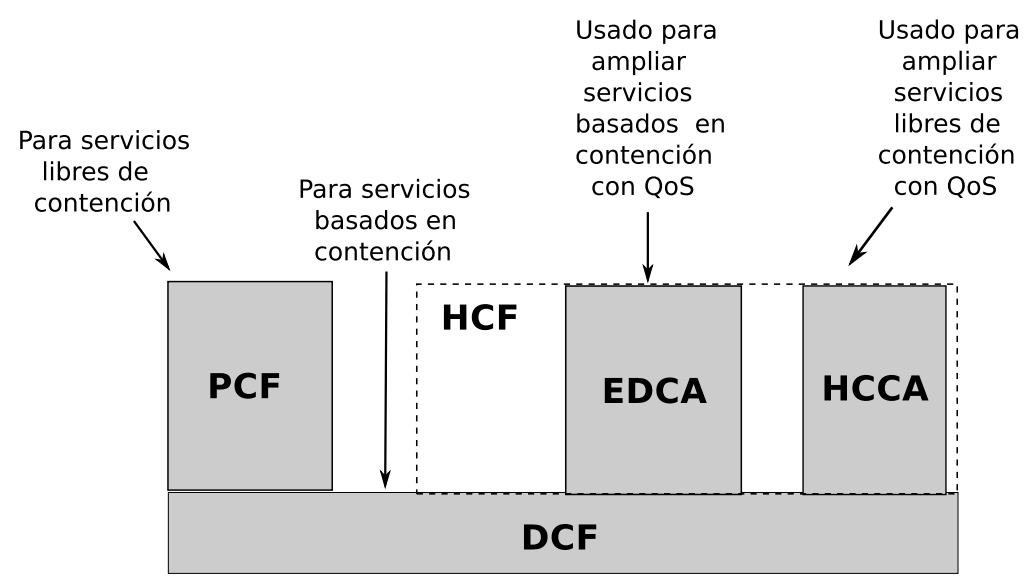

Figura 4.2: Funciones de acceso al medio en el estándar IEEE 802.11e.

\subsection{Función de acceso al canal controlado por $\mathrm{HCF}$ (HCCA)}

HCCA es una ampliación de PCF para ofrecer QoS. Se puede usar tanto en el intervalo de contención como en el intervalo libre de contención de la super-trama IEEE 802.11e. En HCCA el acceso al canal está basado en el envío de mensajes de polling. Siendo HCCA una función centralizada. El HC, función que normalmente es desempeñada por el AP para QoS (QAP), es el que decide a que estación le toca transmitir. Para ello, el HC envía mensajes

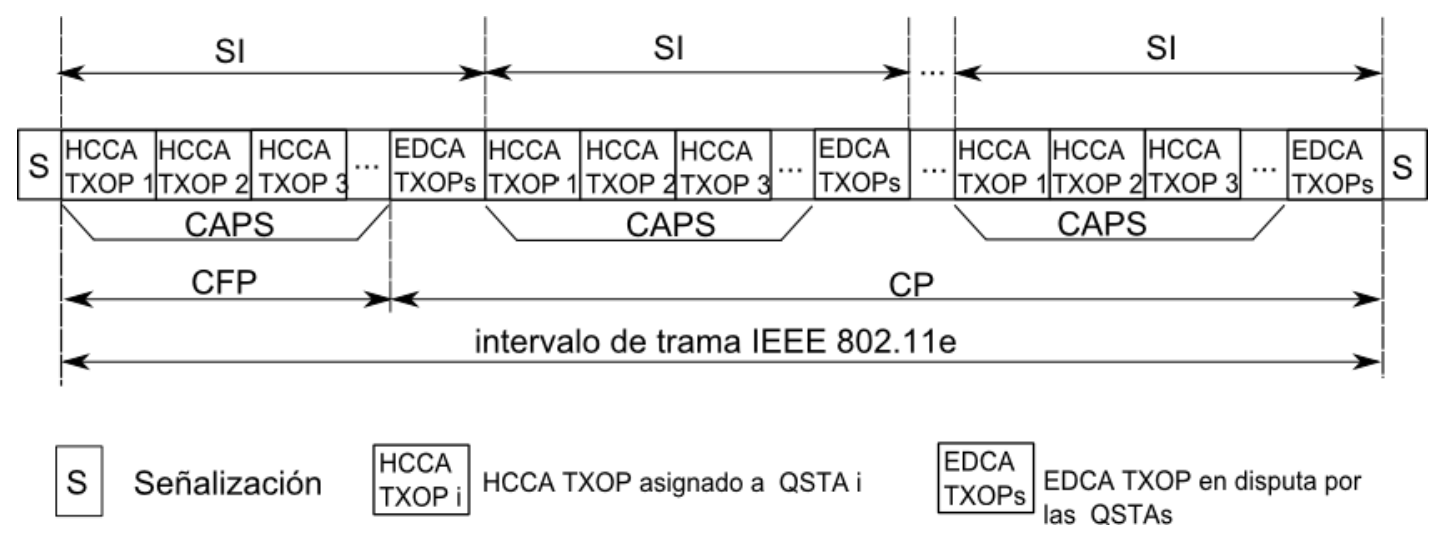

Figura 4.3: Acceso al medio mediante HCCA. 
de polling a las estaciones que tiene asociadas. HCCA es una función que busca garantizar los requisitos de QoS estrictos.

En la Figura 4.3 se muestra el mecanismo de elección controlado por el coordinador híbrido ubicado en el QAP. El HC gestiona el ancho de banda, incluyendo la asignación de oportunidades de transmisión (TXOP) a los nodos inalámbricos a los que ofrece QoS (QSTAs). Una TXOP que se obtiene usando el acceso al cana basado en contención, se denomina EDCATXOP. Mientras que una TXOP garantizada a través de HCCA se denomina HCCA-TXOP. La duración de la EDCA-TXOP se transmite a las QSTAs en la tramas de señalización junto a otros parámetros relacionados con EDCA. QAP puede iniciar varias ráfagas de intervalos libres de repetición, llamados períodos de acceso controlados (CAPs), usando HCCA en cualquier momento después de detectar que el canal se encuentra libre por un intervalo de PIFS. Como PIFS es más corto que DIFS o AIFS, un QAP tiene más probabilidades de iniciar HCCA que EDCA. HCCA es más flexible que PCF, debido a que el acceso al canal mediante CAPs sólo es posible durante el período CFP en PCF.

\subsection{Acceso coordinado y distribuido mejorado (EDCA)}

EL mecanismo EDCA, como evolución de DCF para IEEE 802.11, incluye todos los elementos básicos de DCF como el protocolo CSMA/CA, el mecanismo de backoff o los espacios inter-trama para la gestión del acceso al canal. Aparte de estos elementos básicos comunes, añade nuevos componentes que permiten introducir QoS en el sistema, como son el concepto de TXOP o el de espacio inter-trama AIFS. EDCA proporciona servicios de tráfico diferenciado, sin reserva de recursos. Por tanto, la asignación de la oportunidad de transmisión se toma basada en decisión por consenso, es decir, la decisión para asignar la TXOP se toma conjuntamente entre todas las estaciones.

El procedimiento estándar para ofrecer QoS en el mecanismo de acceso al medio EDCA en IEEE 802.11e consiste en la modificación de uno de los siguientes parámetros a nivel de la capa MAC:

- El intervalo de Backoff (BI)

- El tamaño de la ventana de contención $(C W)$

- El espacio inter-trama arbitrario (AIFS).

Los mecanismos de BI y CW están diseñados para controlar el proceso de backoff por medio de una elección adecuada de los parámetros para diferentes clases de tráfico. Por su parte, AIFS tiene una función equivalente al espacio inter-trama distribuido (DIFS) del mecanismo de acceso al medio en el estándar IEEE802.11; la diferencia radica en que puede haber un valor distinto de AIFS por cada AC. El tiempo AIFS es el intervalo de tiempo mínimo que tiene que estar el medio libre antes de empezar a transmitir.

En esencia, el procedimiento de backoff opera de modo tal que en promedio, a una AC con la más alta prioridad le son asignados el menor intervalo de contención y valor AIFS. Esto 
asegura que las tramas de datos de alta prioridad tengan la menor demora en el acceso al canal. Por consiguiente, obtienen una mayor oportunidad de acceder al medio que las tramas de baja prioridad.

Cada categoría de acceso dispone de su propia cola de transmisión caracterizada por los parámetros de acceso AIFS, CW y TXOP. En el caso que una estación opere simultáneamente con distintas AC y el tiempo aleatorio de backoff termine en el mismo instante, la categoría con la prioridad más alta es la que empieza a transmitir antes, mientras que el resto se comporta como si se hubiera producido una colisión al acceder al medio. A este tipo de evento se le denomina colisión virtual.

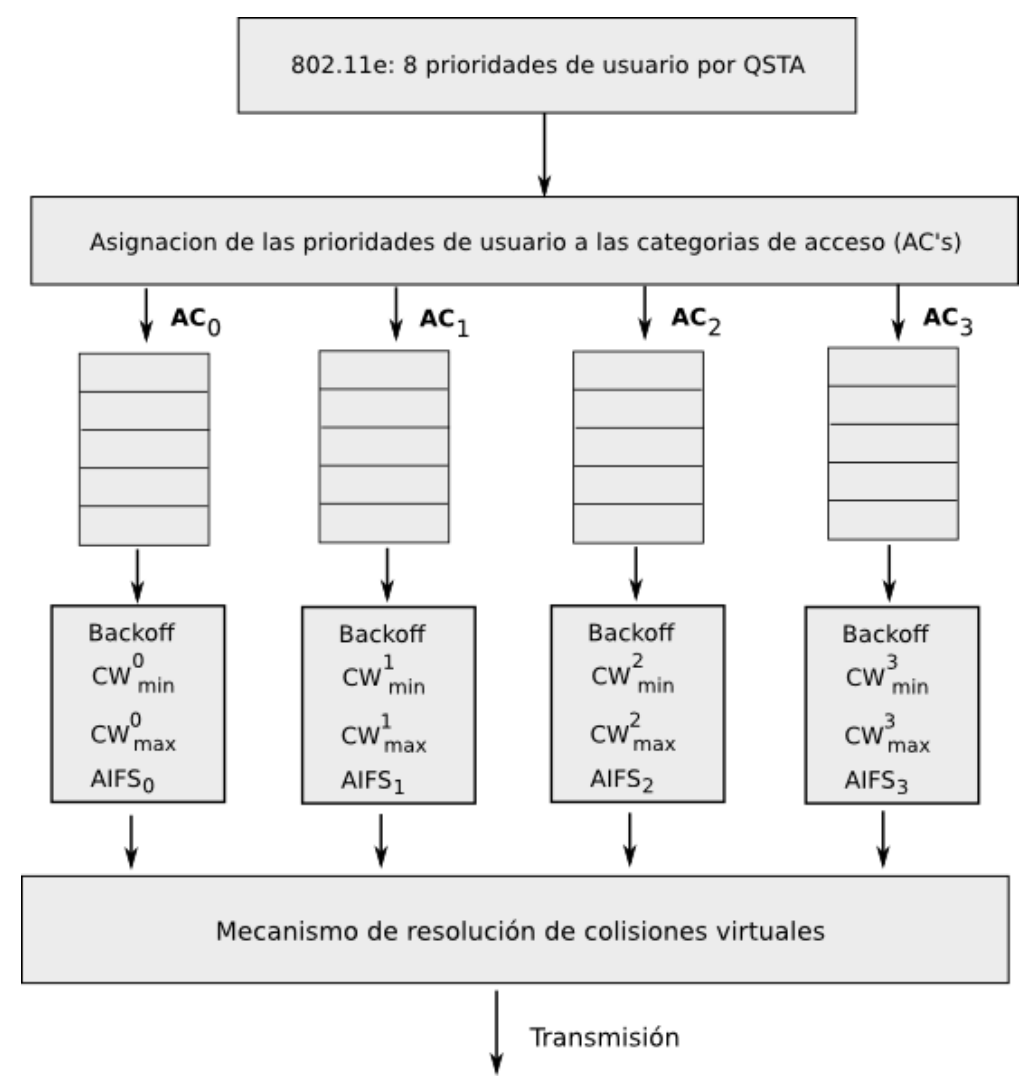

Figura 4.4: Esquema de la función de acceso al medio EDCA.

\subsection{Limitaciones de QoS en IEEE 802.11e}

Existen algunas características en el diseño de las funciones HCCA y EDCA que, bajo ciertas condiciones, tienden a actuar en detrimento de las garantías que el estándar ofrece en cuanto a requerimientos de QoS para las aplicaciones y servicios multimedia. 


\subsubsection{Limitaciones en HCCA.}

HCCA garantiza que las aplicaciones con límites estrictos en tiempo puedan cumplir con sus requerimientos en el caso del tráfico con tasa de bit constante (CBR) haciendo uso del planificador round-robin de referencia. Sin embargo, no tiene control sobre el retardo que puedan experimentar los flujos con tasa de bit variable. Esto debido a que las constantes fluctuaciones en las tasas de transmisión de los flujos de tráfico de tasa variable tienden a rebasar la estimación sobre la tasa promedio, por lo que los paquetes permanecen en la cola de salida por más tiempo incrementando con ello el retardo de extremo a extremo. Por otra parte, si las tasas de trasmisión pico por tipo de aplicación son reportadas al QAP y son usadas para calcular las TXOPs, las TXOPs serán lo suficientemente grandes para permitir la entrega de este tipo de paquetes, y el retardo de las aplicaciones puede ser controlado. No obstante, un menor número de flujos de tráfico puede ser admitido en comparación con el caso donde se emplean los valores promedio para calcular las TXOP, en cuyo caso el canal podría ser subutilizado. Esta deficiencia en HCCA se origina por la asignación de todas las TXOPs sobre el mismo periodo de servicio, sin tomar en cuenta que los diferentes tipos de tráfico pueden generar ráfagas de información a diferentes frecuencias. Por ello, la investigación en el área esta orientada al diseño de algoritmos planificadores que actúen en forma adaptativa a las fluctuaciones en las tasas de transmisión.

Adicionalmente como se reporta en [12], HCCA fue desarrollada explícitamente para redes inalámbricas en modo infraestructura (ó WLAN), por lo que carece de la portabilidad necesaria para extender su funcionamiento a una configuración ad-hoc de los dispositivos de red.

\subsubsection{Limitaciones en EDCA.}

En EDCA, la clasificación de los paquetes por AC es sólo para los flujos de tráfico internos, por lo que no proporciona mecanismos para gestionar la prioridad entre las transmisiones provenientes de diferentes estaciones dentro del área de servicio de la red. Este inconveniente puede conducir a los siguientes problemas:

- Acceso Paralelo por AC: Esta situación se presenta cuando paquetes dentro de la misma AC son transmitidos concurrentemente en la WLAN por varias estaciones, esto ocasiona largos retardos de contención, lo que deteriora el desempeño obtenido por EDCA.

- Asignación estática de AIFS: Para explicar esta deficiencia en EDCA, debe observarse que cuando las clasificaciones de acceso (ACs) para el tráfico de alta prioridad se encuentran inactivas, las clases de tráfico de baja prioridad aún requieren esperar largos periodos AIFS antes de que el proceso de backoff comience la cuenta a cero. Como resultado el throughput agregado podría verse afectado.

- Diferenciación intra-AC: EDCA no proporciona mecanismos para que cada estación pueda saber acerca del estado de las otras estaciones. Entonces, cuando múltiples entidades con la misma prioridad AC pertenecientes a diferentes estaciones eligen los 
mismos valores de backoff. Esto inevitablemente conduce a un incremento en el número de colisiones.

Adicionalmente a lo arriba expuesto, el procedimiento de backoff para determinar el periodo de contención debe ajustarse a la carga de tráfico. Esto a fin de evitar la incertidumbre en el desempeño del procedimiento MAC. 


\section{Capítulo 5 Estado del arte}

En la Tabla 5.3 [11] se resumen algunos de los trabajos recientes más representativos que buscan dotar de mecanismos de QoS a las redes IEEE 802.11. Una característica común entre esos artículos, es que basan sus propuestas en mejorar el soporte de QoS para redes en el estándar IEEE 802.11 en presencia de tráfico multimedia en tiempo real. Como parámetro común para ofrecer sustento a los resultados obtenidos, se realiza una evaluación de desempeño mediante simulación entre la propuesta y el rendimiento de IEEE 802.11 e IEEE 802.11e en términos de utilización del ancho de banda, tasa de transmisión, retardo, jitter, tasa de errores y el número de estaciones dentro del BSA.

Si bien, los trabajos sobre QoS en 802.11 proponen soluciones desde diferentes aproximaciones, puede reconocerse un patrón común a cada uno de ellos. En [10], los autores presentan estas posibles direcciones para garantizar QoS en IEEE 802.11:

- Diferenciación de servicios

- Extracción de información basada en la capa PHY

- Reservación de recursos y control de admisión en la capa MAC

- Ajuste de parámetros IEEE 802.11.

Los trabajos revisados corresponden a combinaciones de dos o más de estas categorías. En especial, en varios trabajos $[3,13,14,15]$ puede destacarse que las propuestas que modifican el algoritmo binario exponencial generador del tiempo de backoff ofrecen características deseables, las cuales son:

- Diferenciación de servicios

- Control de admisión

- Equidad en medios egoístas

- Optimización de la tasa de transmisión sobre condiciones de saturación de la red

- Facilidad de implementación en los esquemas MAC existentes. 
Una vez definidos los aspectos de IEEE 802.11e susceptibles a ser mejorados, se tomaron como base el trabajo presentado en $[2,3]$. A partir del razonamiento planteado en estos trabajos, identificamos aspectos en los que era posible mejorar y que se integraron en nuestra propuesta.

\subsection{Algoritmo RAMPS-EDCA}

RAMPS [2] busca minimizar las altas tasas de colisión observadas en EDCA durante los periodos con altas cargas de tráfico sobre la red. El algoritmo está basado en la selección aleatoria de dos parámetros dinámicos de QoS: CW y AIFS. CW y AIFS desempeñan un importante papel en la determinación del tiempo que una estación tiene que esperar antes de iniciar una transmisión (periodo de backoff). Introduciendo desplazamientos aleatorios de forma dinámica en ambos parámetros, se busca evitar que las distintas entidades dentro de una misma $\mathrm{AC}$ seleccionen los mismos valores durante el período diferido de acceso al canal. RAMPS consta de dos etapas: la etapa de selección de la ventana de contención y la etapa de selección del valor AIFS.

\subsubsection{Etapa de selección de la ventana de contención}

En esta etapa, el desplazamiento aleatorio sobre el período de backoff se determina en base al valor por defecto para CW según el estándar [1] y considerando la actual tasa de pérdida $a(i)$ por AC de la estación. El uso de la tasa de pérdida se justifica debido a que muestra el hueco entre la tasa de bit de la aplicación y el throughput del medio físico, indicando con ello el estado actual de QoS que la red ofrece [12]. Como se muestra en la siguiente ecuación, el parámetro $a(i)$ es calculado como la proporción del número de paquetes descartados $L$ al número de paquetes enviados $S$ por cada AC sobre un período de tiempo.

$$
a(i)=\frac{L}{S}
$$

El procedimiento para adaptar el valor de CW acorde a las condiciones de la red mediante $a(i)$ se presenta a continuación. El valor máximo para el desplazamiento $F_{\max }$ es calculado como:

$$
F_{\text {max }}(i)=[\mathrm{CW}(i)+1]^{a(i)}
$$

Donde el valor actual para el desplazamiento $F(i)$ es seleccionado aleatoriamente dentro del intervalo $\left[0, F_{\max }(i)\right]$.

$$
F(i)=\operatorname{rand}\left[0, F_{\max }(i)\right]
$$

El valor de $F(i)$ se selecciona acorde al estado de la red para determinar el período total de backoff. Por ejemplo, si la red se encuentra congestionada, el valor de $F(i)$ deberá ser 
grande y por consiguiente provocará un incremento en el número posible de valores para el desplazamiento aleatorio. Esto reduce la probabilidad de que varias entidades dentro de la misma AC seleccionen el mismo valor para el desplazamiento aleatorio. Entonces, el periodo total de backoff $(T)$ puede obtenerse como:

$$
T=\operatorname{rand}[1, C W(i)+1]+F(i)
$$

Añadiendo el valor del desplazamiento $F(i)$ al valor aleatorio de backoff se incrementa el grado de aleatoriedad por un factor de $[1+F(i)$, CW $+1+F(i)]$, y también se proporciona diferenciación de prioridades del tráfico perteneciente a la misma clase así como entre el tráfico proveniente de diferentes clases. Como es improbable que el valor de CW para las clases de alta prioridad sea tan alto como el de las clases de baja prioridad, RAMPS proporciona una buena diferenciación inter-AC. La Figura 5.1 ilustra el nuevo método de acceso propuesto para EDCA con la actualización dinámica sobre los valores de CW en base a la tasa de pérdida por AC en cada estación.

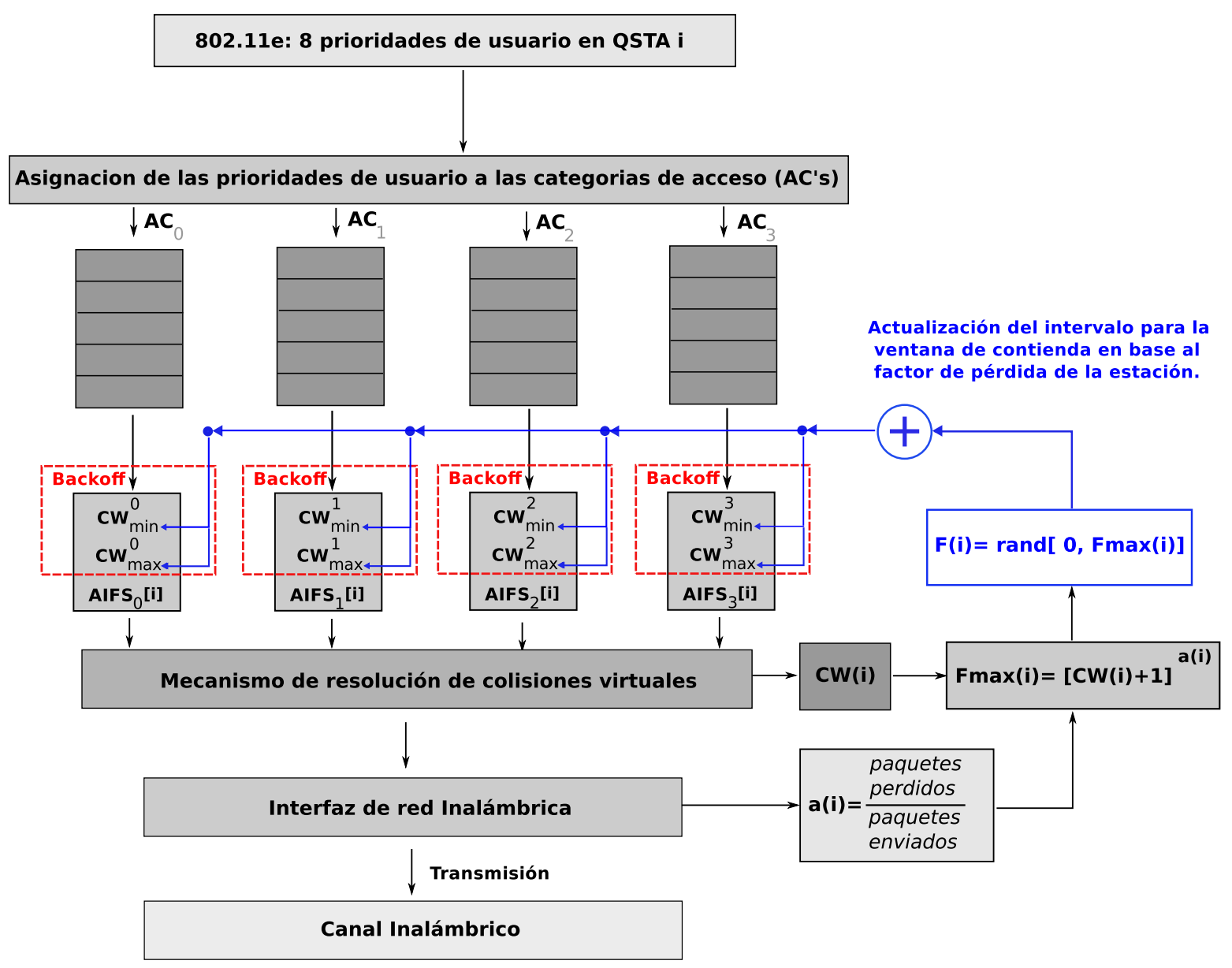

Figura 5.1: Diagrama de la estructura propuesta para el acceso al canal mediante EDCA. 


\subsubsection{Etapa de selección del valor AIFS}

Adicionalmente a la diferenciación inter-AC basada en el valor de CW descrita anteriormente, RAMPS usa un esquema basado en AIFS para obtener una mayor discriminación sobre el tráfico dentro de una misma AC proveniente de diferentes estaciones. Esta técnica sirve de apoyo al esquema basado en CW para evitar que dos o más AC dentro de la misma categoría de prioridad seleccionen el mismo valor de backoff. Un grado adicional de diferenciación puede obtenerse a través del valor usado para AIFS. En EDCA el proceso para asignar el valor de AIFS por AC se obtiene según la ecuación 5.5.

$$
\operatorname{AIFS}(\mathrm{AC})=\mathrm{SIFS}+\operatorname{AIFSN}(\mathrm{AC}) \times \text { SlotTime }
$$

Donde SIFS se refiere al espacio inter-trama con el menor tiempo de espera que es usado para mensajes cortos, y AIFSN es el número de ranuras de tiempo después de SIFS. Una estación deberá esperar un tiempo SIFS antes del inicio de una transmisión o antes de realizar la invocación al procedimiento de backoff. RAMPS extiende el uso del valor de AIFS para proporcionar diferenciación intra-clase adicionalmente a la diferenciación por AC. Esto se consigue al asignar un intervalo de valores $[N, M]$ para $A I F S N$ por cada AC en lugar de un único valor estático como se utiliza en EDCA [1] (una ranura de tiempo para transmisiones de alta prioridad y dos ranuras de tiempo para transmisiones de baja prioridad). El rango de valores de $A I F S N$ para cada $\mathrm{AC}$ está definido y el valor de $A I F S N(i)$ es único. $A \operatorname{IFSN}(i)>A \operatorname{IFSN}(i+1)$, donde $\operatorname{AIFSN}(i)$ es el valor para una AC de alta prioridad y $A I F S N(i+1)$ es el valor correspondiente para la $\mathrm{AC}$ de baja prioridad. Cada vez que una AC entra en un periodo de contención seleccionará un valor aleatorio dentro del rango de $A I F S N$. Este valor se añade como un desplazamiento sobre el valor previo de $A I F S N$. El valor resultante es entonces usado como el actual valor de $A I F S N$.

$$
A I F S N_{\text {new }}(\mathrm{AC})=A I F S N_{\text {old }}(\mathrm{AC})+A I F S N_{\text {offset }}(\mathrm{AC})
$$

Donde

$$
A I F S N_{o f f s e t}(\mathrm{AC})=\operatorname{rand}[N, M]
$$

Como se observa en la Ecuación 5.6, el valor aleatorio del desplazamiento seleccionado siempre es añadido al valor previo de $A I F S N$ ( $A I F S N o l d$ ) para obtener el valor actual para AIFSN (AIFSNnew). La adición del valor aleatorio del desplazamiento al valor previamente obtenido incrementa el grado de aleatoriedad del esquema. Resulta evidente que si el nuevo valor de $A I F S N$ no es limitado, este conducirá infinitamente a valores cada vez mayores. Para evitar que esto ocurra, se fija un valor máximo y uno mínimo que limitan los posibles valores que pueda tomar $A I F S N$ (véase la tabla 5.1 [2]). La probabilidad de tener dos entidades $\mathrm{AC}$ dentro de la misma categoría y con los mismos valores AIFSNold y $A I F S N o f f s e t$ es muy reducida debido especialmente a que tanto el valor de AIFSNold y el de $A I F S N$ of $f$ set se obtienen a partir de procesos aleatorios independientes. 


\begin{tabular}{|c|c|c|}
\hline Prioridad & $N$ & $M$ \\
\hline$A C_{0}$ & 1 & 5 \\
\hline$A C_{1}$ & 5 & 8 \\
\hline$A C_{2}$ & 5 & 8 \\
\hline$A C_{0}$ & 8 & 11 \\
\hline
\end{tabular}

Tabla 5.1: Rango de desplazamiento para los valores de AIFSN por AC.

\subsection{Algoritmo CI-EDCA}

En [3] se presenta un nuevo mecanismo de acceso compatible con el modo EDCA del estándar IEEE 802.11e, en el que la asignación de la TXOP se obtiene en base a un esquema de prioridades inter-estación basado en conteo de tráfico local. Esto permite la transmisión de los paquetes basada no sólo en la prioridad de la AC a la que pertenecen, sino también en la capacidad efectiva del canal en base al historial de transmisión por nivel de prioridad de la estación. El mecanismo de clasificación intra-nodo propuesto consta de la modificación en la regla de acceso al canal para EDCA, que opera en conjunto con una nueva clasificación tolerante a prioridad basada en CW que busca optimizar el rendimiento para las AC inferiores. El mecanismo propuesto en [3] para CI-EDCA consta de dos etapas, cada una orientada a resolver uno de los principales problemas que presenta el actual modo EDCA.

\subsubsection{Esquema modificado de acceso EDCA para QoS}

Para resolver el problema del acceso paralelo por AC, Se considera la información del historial de tráfico local vinculado a la estación. Para ello, en cada uno de los nodos de la red un contador local $\left(S_{i}\right)$ lleva la cuenta de los paquetes transmitidos por cada AC. Este nuevo parámetro proporciona un nuevo identificador inter-estación $j$ que permite distinguir al flujo de tráfico proveniente de una estación en particular de los otros flujos de tráfico dentro de la misma categoría de acceso. El nuevo valor AIFS $[i][j]$ no se mantendrá fijo, en vez de ello se ajustará dinámicamente entre los valores $\operatorname{AIFS}[i][j-1]$ y $\operatorname{AIFS}[i][j+1]$ dependiendo del valor actual de $S_{i}$. Entonces, la nueva clase móvil $j$ proporciona al paquete un distinto nivel de importancia relativa dentro del mismo nivel de transmisión AC. Se tienen dos consecuencias del esquema propuesto: primero, los flujos de tráfico equivalentes dentro de la misma AC no tendrán la misma prioridad de acceso al canal debido a la nueva configuración de AIFS. Segundo, disminuirán los intentos simultáneos de transmisión intra-AC. Como resultado, el periodo de contención debido a intentos paralelos de acceso al canal se reduce de forma considerable para transmisiones similares dentro de una misma AC, y el retardo inducido por las colisiones intra-AC también disminuye sustancialmente. El nuevo valor AIFS $[i][j]$ resulta más significativo en términos del manejo de tráfico, mientras mantiene la estricta clasificación existente entre las AC como se muestra en la Ecuación 5.7.

$$
\operatorname{AIFS}[i][0]<\ldots<\operatorname{AIFS}[i][j]<\ldots<\operatorname{AIFS}[i][n-1]
$$


para

$$
\begin{aligned}
& \operatorname{AIFS}[i-1][n-1]<\operatorname{AIFS}[i][0] \\
& \operatorname{AIFS}[i][n-1]<A I F S[i+1][0]
\end{aligned}
$$

con

$$
\begin{aligned}
& i=\{0, \ldots, 3\}: \text { categoría de tráfico. } \\
& j=\{0, \ldots, n-1\}: \text { clasificación por actividad de la estación. }
\end{aligned}
$$

La Figura 5.2 ilustra el nuevo método de acceso al canal adoptado por el nuevo modelo EDCA. De acuerdo a la descripción del nuevo modelo EDCA, cuando ocurre la transmisión de una trama $A C[i]$, la estación emisora con clasificación de prioridad $j$ usa el nuevo valor $A I F S[i][j]$ para el siguiente acceso al canal de acuerdo a la Ecuación 5.7. Los valores para $C W[i]$ no se ven afectados por el nuevo arreglo debido a que éste parámetro depende únicamente de la categoría de tráfico $i$ en particular.

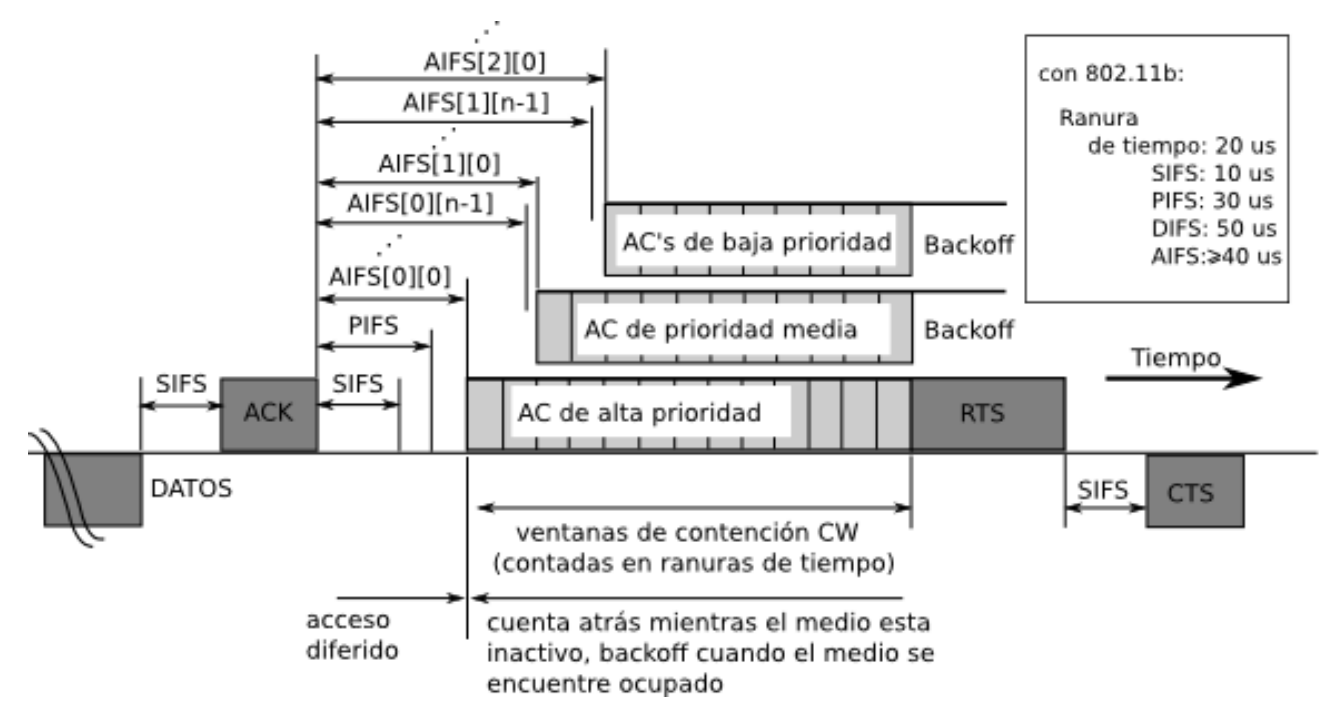

Figura 5.2: Relación inter-trama modificada para EDCA

La Figura 5.3 ilustra el procedimiento de asignación dinámica de prioridades basado en la clasificación de la estación por actividad, el cual se explica a continuación:

- Valor del factor de decisión $(k)$. Cuando es alcanzado por uno de los contadores de tráfico de paquetes $\left(S_{0}, S_{1}, S_{2}, S_{3}\right)$, la estación deberá permutar a otro estatuto de prioridad inter-nodo acorde al patrón de tráfico adoptado.

- Si uno o ambos de los contadores $S_{0}$ y $S_{1}$ alcanza el valor de $k$. Se concluirá que la actual estación tiene importantes flujos multimedia por transmitir. Entonces se le debe asignar una mayor prioridad sobre otras estaciones de la red. Por tanto, se traslada de su actual clase $j$ a otra categoría $(j-1)$ más elevada. 
- Por su parte, cuando los contadores $S_{2}$ o $S_{3}$ alcanzan el valor $k$, la clase $j$ de la estación conmuta a una categoría inferior $j+1$. En consecuencia, se puede inferir que las transmisiones de la estación corresponden a las categorías de tráfico de baja prioridad.

- Solo el contador de tráfico $S_{i}$ responsable de la permutación entre clases se reiniciará a cero.

- La clase inicial $j$ y el contador $S_{i}$ tendrán respectivamente los valores de $(n-1)$ y cero para cada una de las estaciones antes de iniciar transmisiones. Entonces, los nodos móviles deberán usar AIFS $[i][n-1]$ para su primera transmisión en la categoría de tráfico $i$.

\subsubsection{Esquema mejorado para la asignación de prioridad basado en CW}

En EDCA, el problema de la inactividad en las categorías de acceso de alta prioridad provoca que las categorías de tráfico inferiores aún tengan que esperar intervalos AIFS prolongados antes de empezar a decrementar su contador de backoff. Sin embargo, el parámetro AIFS $[i]$ proporciona una clasificación de prioridad estricta entre las categorías de tráfico. En consecuencia, se elige manipular el parámetro CW $[i]$ para minimizar el retardo en la espera y entonces maximizar el throughput agregado. Para ello, se establece una prioridad estricta entre $\mathrm{AC}[0]$ y $\mathrm{AC}[1]$. Mientras se establece una ponderación equitativa entre $\mathrm{AC}[2]$ y $\mathrm{AC}[3]$ modificando el rango de valores para el parámetro CW como se describe en la tabla 5.2.

\begin{tabular}{|c|c|c|c|c|}
\hline Prioridad & $A C_{0}$ & $A C_{1}$ & $A C_{2}$ & $A C_{3}$ \\
\hline$C W_{\min }$ & 3 & 7 & 15 & 15 \\
\hline$C W_{\max }$ & 7 & 15 & 1023 & 1023 \\
\hline
\end{tabular}

Tabla 5.2: Parámetros EDCA usados en la implementación de la propuesta [3].

El esquema anterior busca remediar la ineficiencia causada por una clasificación estricta en la regla de acceso EDCA, mientras mantiene la estructura original de prioridades entre las categorías de tráfico. Como sólo se modifica el rango de valores posibles para CW[2] y CW[3], en esencia, no se modifica el algoritmo estándar para IEEE 802.11e. 


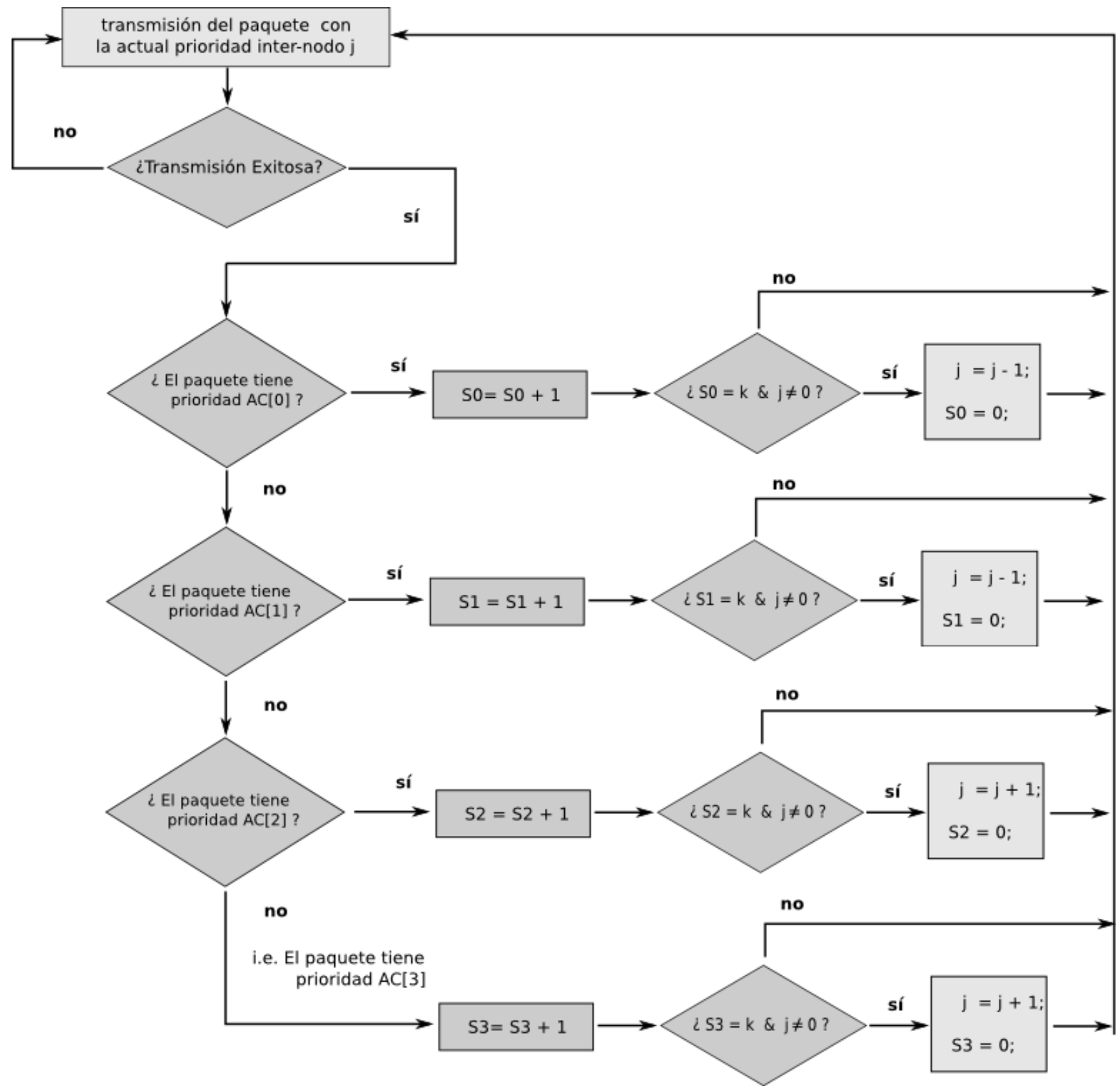

Figura 5.3: Nuevo esquema de clasificación inter-nodo basado en la actividad de la estación para EDCA. 


\begin{tabular}{|c|c|c|}
\hline $\begin{array}{l}\text { Título del } \\
\text { artículo }\end{array}$ & Mejora sobre el estándar & Posibles deficiencias \\
\hline WTN $[16,17]$ & $\begin{array}{l}\text { - Disminuye el costo por transmisión y el número de } \\
\text { colisiones } \\
\text { - Resuelve el inconveniente en el estándar 802.11e que } \\
\text { suprime el tráfico de mejor esfuerzo cuando se tiene } \\
\text { una gran cantidad de estaciones en un BSA. }\end{array}$ & $\begin{array}{l}\text { El compromiso entre el requisito de QoS de } \\
\text { la aplicación y la conectividad para el tráfico } \\
\text { de baja prioridad no está optimizado. }\end{array}$ \\
\hline $\begin{array}{l}\text { QoS provisioning } \\
\text { system for multime- } \\
\text { dia transmission in } \\
\text { IEEE } 802.11 \text { Wireless } \\
\text { LAN [13] }\end{array}$ & $\begin{array}{l}\text { - Brinda soporte para la transferencia de llamadas o } \\
\text { sesiones de datos entre BSA contiguos } \\
\text { - Ofrece garantías para que las fuentes de tráfico en } \\
\text { tiempo real satisfagan sus restricciones de tiempo } \\
\text { - Implementa una política de petición/rechazo de } \\
\text { nuevas peticiones de transmisión. }\end{array}$ & $\begin{array}{l}\text { El AP compite por el acceso al medio por lo } \\
\text { que es posible que se induzcan retardos en el } \\
\text { inicio de futuras transmisiones. }\end{array}$ \\
\hline $\begin{array}{l}\text { Reservation } \begin{array}{r}\text { and } \\
\text { grouping }\end{array} \text { stations } \\
\text { for the IEEE } 802.11 \\
\text { DCF [14] }\end{array}$ & $\begin{array}{l}\text { - Cuando el número de estaciones en un BSA se in- } \\
\text { crementa, el esquema propuesto reduce el número } \\
\text { de colisiones entre las estaciones activas mejorando } \\
\text { el desempeño del sistema respecto a DCF y EDCA. }\end{array}$ & $\begin{array}{l}\text { El algoritmo de generación del tiempo de es- } \\
\text { pera es susceptible a ser optimizado. }\end{array}$ \\
\hline $\begin{array}{lr}\text { A new inter-node } \\
\text { priority } r \text { access } \\
\text { enhancement } \\
\text { scheme } \\
\text { for IEEE } 802.11 \\
\text { WLANs [3] }\end{array}$ & $\begin{array}{l}\text { - Al detectarse intervalos largos de inactividad en los } \\
\text { flujos de alta prioridad se eleva la prioridad de los } \\
\text { flujos de las categorías más bajas, para evitar la } \\
\text { degradación en la tasa de transmisión } \\
\text { - Resuelve el inconveniente en IEEE 802.11e en la } \\
\text { degradación de la tasa de transmisión debido a la } \\
\text { reservación estática de los recursos por categoría. }\end{array}$ & $\begin{array}{l}\text { El factor de predicción para conceder el acce- } \\
\text { so al medio entre clases de tráfico equivalen- } \\
\text { tes mantiene la clasificación estricta de tráfi- } \\
\text { co entre clases activas. }\end{array}$ \\
\hline $\begin{array}{l}\text { Enhancing IEEE } \\
802.11 \text { Random } \\
\text { Back off in Selfish } \\
\text { Environments [15] }\end{array}$ & $\begin{array}{l}\text { - Modificando el proceso en el que se genera la venta- } \\
\text { na de contención que inicia el proceso de backoff. Se } \\
\text { implementa políticas de equidad en escenarios don- } \\
\text { de existen estaciones que obtienen mayor prioridad } \\
\text { en el turno de transmisión al deliberadamente mo- } \\
\text { dificar su esquema de acceso al medio compartido. }\end{array}$ & $\begin{array}{l}\text { Conserva el mecanismo de decremento en } \\
\text { la ventana de contención propuesta en el } \\
\text { estándar. Con lo que mecanismos de decre- } \\
\text { mento adaptables a las condiciones del medio } \\
\text { brindarían mejores tasas de transmisión. }\end{array}$ \\
\hline $\begin{array}{l}\text { Enhancing } \quad r \text { IEEE } \\
802.11 \quad \text { MAC in } \\
\text { congested environ- } \\
\text { ments }[18]\end{array}$ & $\begin{array}{l}\text { - Mediante un decremento conservativo sobre la ven- } \\
\text { tana de contención después de una transmisión exi- } \\
\text { tosa. El esquema se adapta a las condiciones del ca- } \\
\text { nal y a las condiciones de competencia de la BSA. }\end{array}$ & $\begin{array}{l}\text { Las condiciones de estado estable óptimo so- } \\
\text { bre la tasa de transmisión para el esquema } \\
\text { propuesto no son consideradas en el presente } \\
\text { análisis. }\end{array}$ \\
\hline $\begin{array}{lr}\text { Adaptive end to end } \\
\text { QoS for } & \text { Multimedia } \\
\text { over r } & \text { Heteroge- } \\
\text { neous } & \text { Wireless } \\
\text { Networks } & 19]\end{array}$ & $\begin{array}{l}\text { - El algoritmo propuesto se adapta al estado del canal } \\
\text { para contener/reanudar las transmisiones en curso } \\
\text { - La toma de decisión a nivel de aplicación resulta en } \\
\text { la obtención de mayores tasas de transmisión } \\
\text { - La característica de adaptación al canal a nivel de la } \\
\text { capa de aplicación brinda la posibilidad de predecir } \\
\text { futuras desconexiones. }\end{array}$ & $\begin{array}{l}\text { Requiere mecanismos de adaptabilidad opti- } \\
\text { mizados al compromiso tasa de transmisión- } \\
\text { QoS en presencia de congestión o perdidas en } \\
\text { el medio inalámbrico. }\end{array}$ \\
\hline $\begin{array}{l}\text { Achieving maximal } \\
\text { VoIP calls in } 802.11 \\
\text { wireless networks }[20]\end{array}$ & $\begin{array}{l}\text { - Capacidad de acceso al canal de forma diferenciada } \\
\text { según los requerimientos de QoS, reduciendo así el } \\
\text { tiempo de transmisión perdido en a la estructura } \\
\text { de asignación de oportunidades de transmisión ori- } \\
\text { ginalmente planteada en IEEE 802.11e. }\end{array}$ & $\begin{array}{l}\text { La sobrecarga debido a las tramas de señali- } \\
\text { zación por el encapsulamiento de la informa- } \\
\text { ción a nivel de la capa MAC puede resultar } \\
\text { significativa. }\end{array}$ \\
\hline
\end{tabular}

Tabla 5.3: Comparación entre las propuestas para QoS en IEEE 802.11. 


\subsection{Descripción de la propuesta}

La Figura 6.1 muestra las características de mayor relevancia en nuestro esquema de clasificación inter-estación para EDCA (ACI-EDCA) basada en la actividad de la estación y en el factor de pérdida.

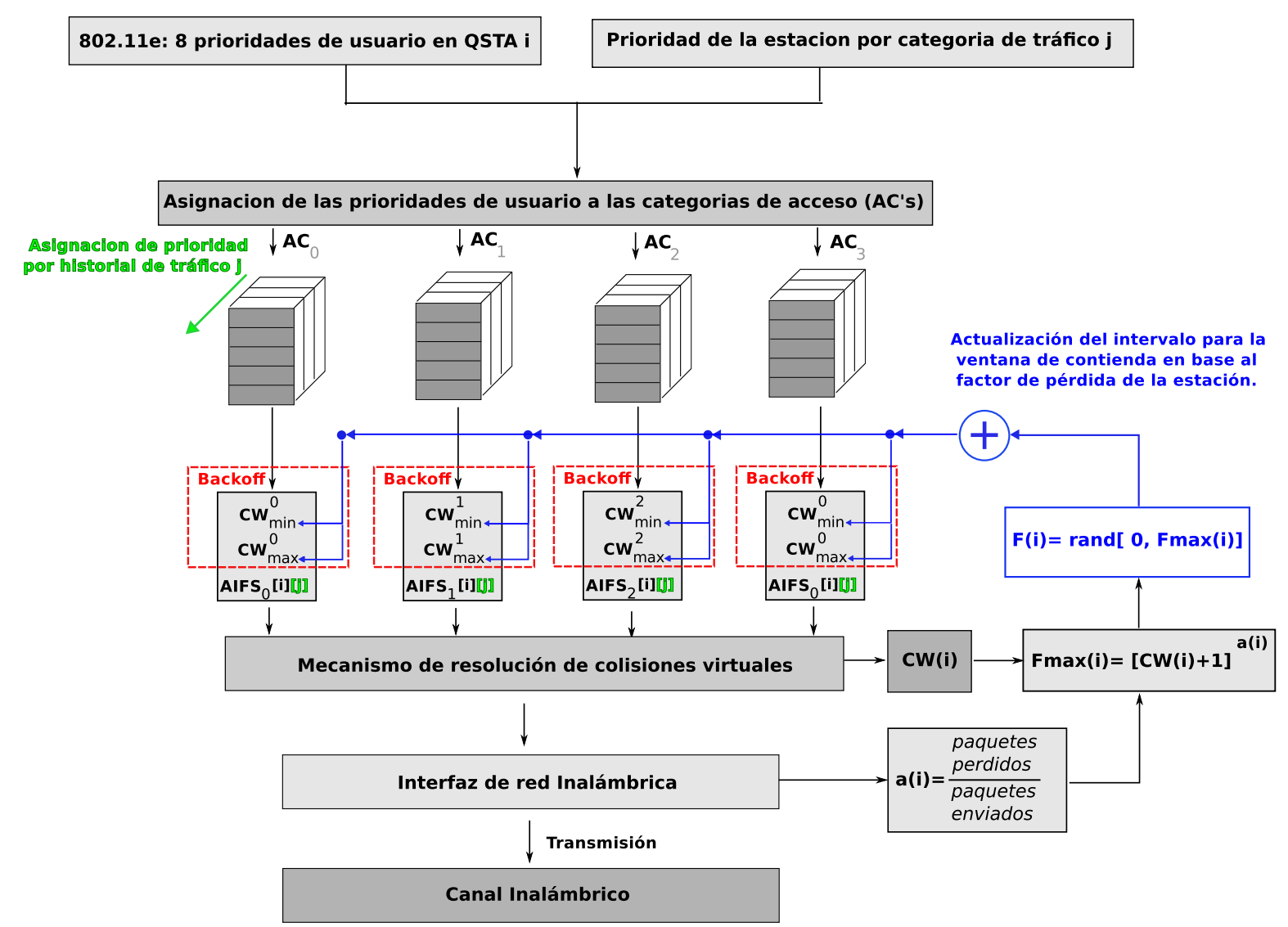

Figura 6.1: Esquema activo de clasificación inter-estación para EDCA. 
El esquema para ACI-EDCA ilustra como el mecanismo dinámico de asignación para el valor del intervalo de contención en base al factor de pérdida propuesto en [2] opera en forma conjunta con el mecanismo de asignación de prioridades basado en la actividad por categoría de acceso planteado en [3].

En el capítulo previo se revisaron los conceptos teóricos que sustentan los trabajos citados $[2,3]$. En base a esta revisión, establecemos una perspectiva sobre el funcionamiento de los algoritmos EDCA modificados que presentan los artículos. En los dos casos, los autores proponen un esquema adaptativo (sobre el rango de valores para el intervalo de backoff [2] o sobre el valor de AIFS [3]) basado en la percepción individual de cada una de las estaciones sobre el estado del medio de transmisión, las condiciones de competencia dentro de la WLAN y la tasa efectiva de transmisión por AC. El objetivo en ambos trabajos, es el establecer una relación entre el algoritmo de acceso al medio basado en prioridades heredado de EDCA con la información obtenida en tiempo real por cada una de las transmisiones efectuadas y/o recibidas por las estaciones. La justificación para este enfoque radica en que dada la naturaleza descentralizada de la función de acceso al medio en el modo EDCA, no existe un mecanismo que permita ajustar la asignación estática para los valores de los parámetros que permiten establecer la prioridad de la categoría de tráfico no sólo respecto al tráfico de otras categorías, sino a la prioridad relativa del flujo de tráfico respecto a otros flujos transmitidos dentro de la misma categoría por otras estaciones dentro de la WLAN (esquema de prioridad inter-estación [2], diferenciación inter-AC [3]).

En cada uno de los artículos, el análisis de resultados presentado muestra claramente una mejora sobre el algoritmo EDCA propuesto en el estándar [1] en cuanto a un manejo más eficiente del medio inalámbrico respecto a los parámetros del retardo, variabilidad del retardo, tasa de bit efectiva y en el decremento en el número de colisiones debido a la asignación simultánea de la TXOP entre las distintas entidades que contienden por el acceso al canal.

No obstante, la justificación en la que los autores ( Wang et al [2], Rebai et al [3]) basan la decisión de implementar un mecanismo auxiliar no adaptativo, como complemento al esquema adaptativo, se basa en suposiciones imprecisas sobre el papel que desempeña el mecanismo de la ventana de contienda durante el periodo de backoff [3] o el del intervalo inter-trama arbitrario [2], que es el lapso de tiempo que cada estación tiene que esperar antes de ejecutar el algoritmo de backoff y antes de iniciar el intento de transmisión según la actual categoría de tráfico. En ambos casos, se propone un mecanismo complementario no óptimo, basado en aumentar la aleatoriedad en la elección del valor actual para AIFS [2] o en disminuir la competencia entre las categorías de tráfico de baja prioridad [3]. Si bien, los resultados demuestran una combinación efectiva de las técnicas adaptativas y las complementarias, en estas últimas se pierde de vista la capacidad de la estación para auto-gestionar el caudal de tráfico inyectado a la WLAN basado en su percepción sobre el estado del medio de transmisión y las condiciones de competencia por AC al interior de la misma.

Una vez establecidos los aciertos y las fallas del enfoque sobre el que basan su estrategia los trabajos previos. El presente trabajo tiene por objetivo obtener aún mejores resultados en cuanto al manejo del tráfico en el modo EDCA para IEEE 802.11e, por ello en el algoritmo 
ACI-EDCA combinamos las técnicas adaptativas que han demostrado su efectividad en trabajos previos, la hipótesis de nuestra propuesta es que mediante un mejor aprovechamiento de la información individual recabada por las estaciones acerca del estado del medio común de transmisión y sobre las condiciones de competencia al interior de la WLAN, la decisión conjunta en la asignación de la TXOP tomará en cuenta las actuales condiciones que la red ofrece para la transmisión de tráfico multimedia, especialmente relevante para los propósitos de nuestra investigación.

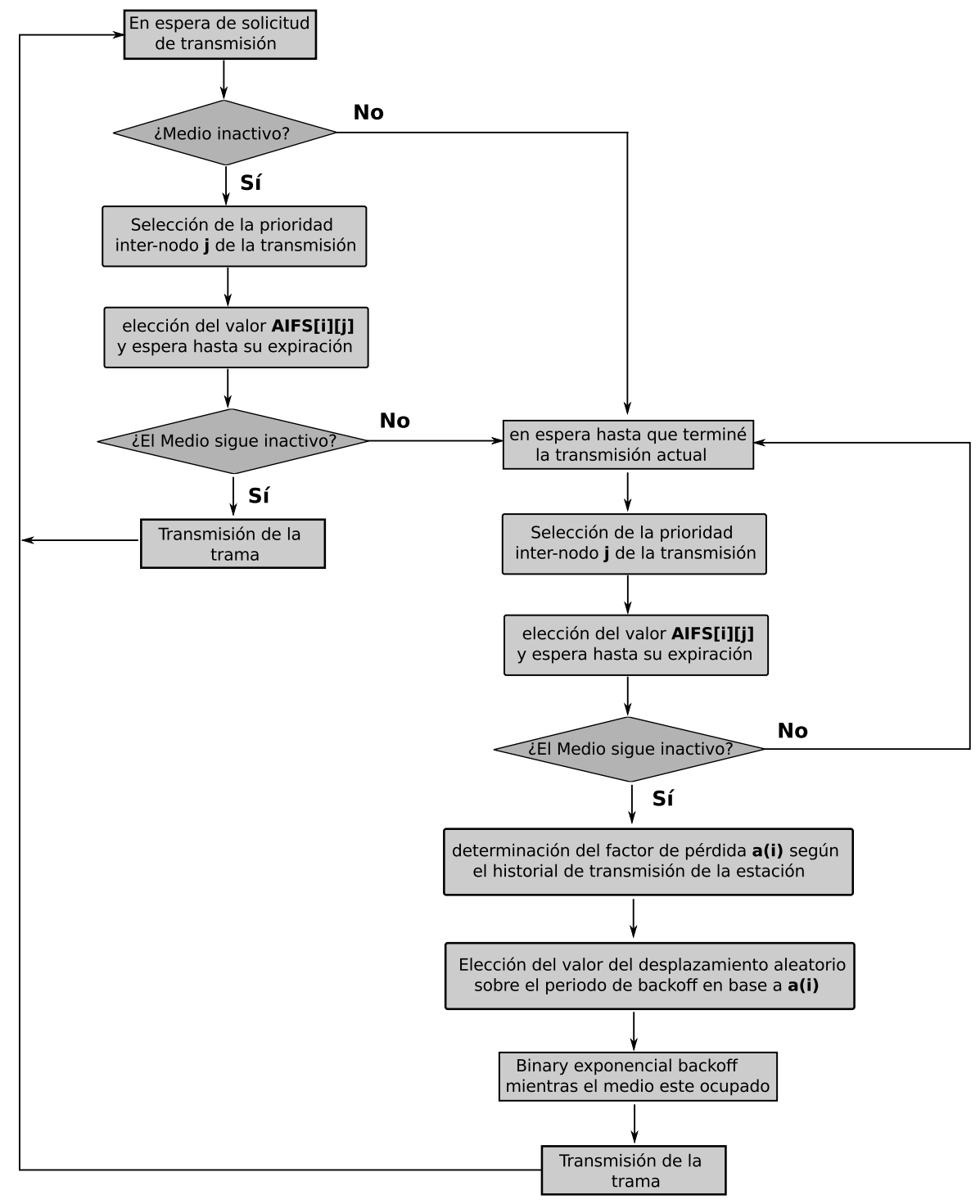

Figura 6.2: Lógica de acceso al medio propuesta.

El algoritmo 1 corresponde al método dinámico de actualización para el valor de AIFS. 
Nótese que la cantidad de nuevas categorías móviles para establecer la actividad de la estación depende exclusivamente del valor asignado a la variable max traffic prority. Conforme a lo reportado en [3] el valor óptimo para esta variable es 6.

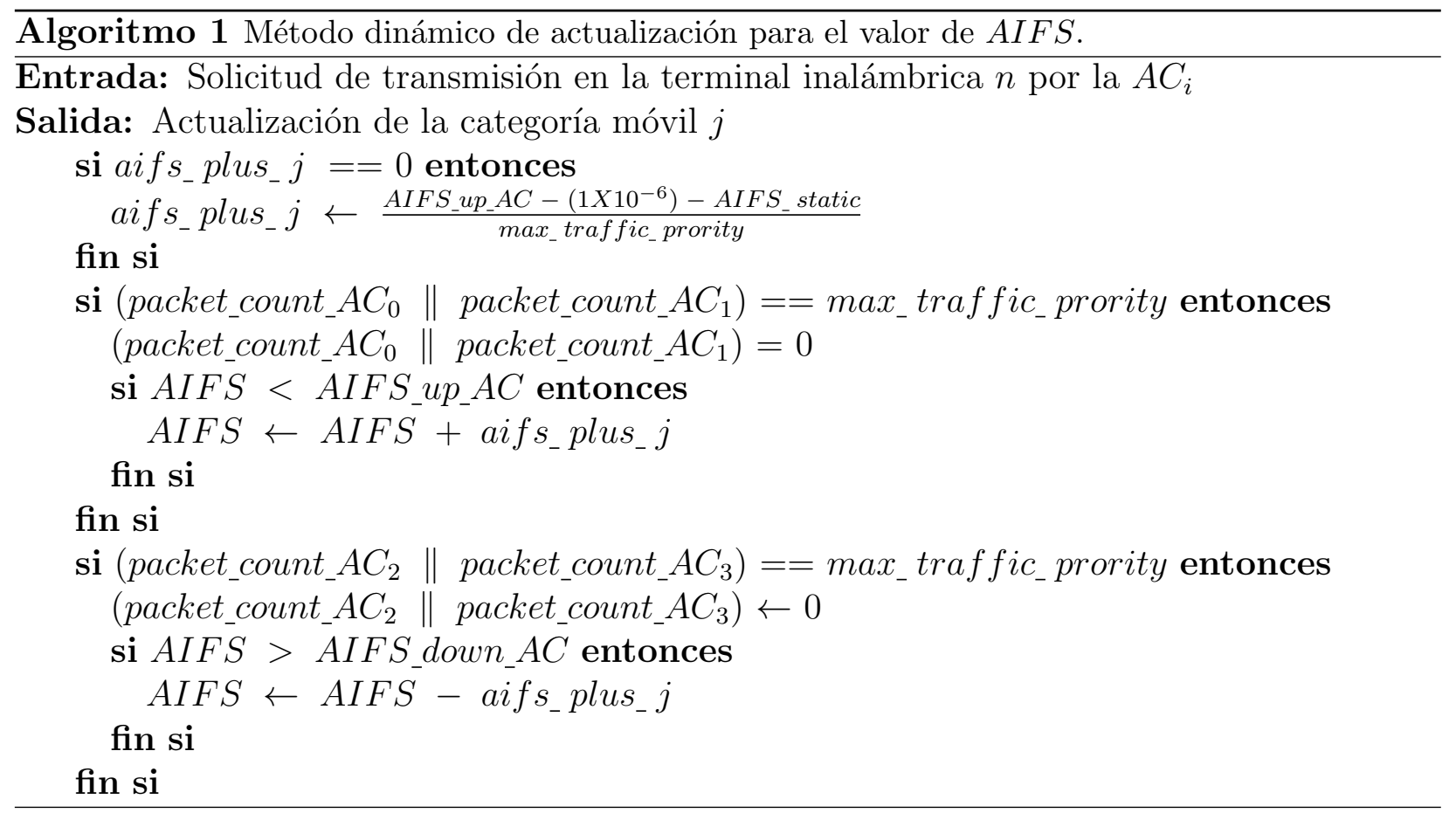

Una aspecto a considerar en la implementación de nuestra propuesta, es que debido a que los autores no indican explícitamente el mecanismo de actualización para el parámetro de la tasa de pérdida en el algoritmo RAMPS [2],hemos adaptado para tal fin, el enfoque propuesto para la actualización en la tasa de colisiones en el algoritmo AEDCA propuesto en [21]. En base a ello, la actualización para el factor de la tasa de pérdida se puede obtener a partir de la Ecuación 6.1.

$$
a(i)_{a v g}=(1-\alpha) \cdot a(i)+\alpha \cdot a(i-1)_{a v g}
$$

Donde $i$ se refiere al $i$-ésimo período de medición y $\alpha$ es un factor de amortiguamiento en el rango de 0 a 1.

El algoritmo 2 muestra la técnica adoptada para la actualización dinámica del valor de la ventana de contención. 
Algoritmo 2 Método dinámico de actualización para los valores de $C W$ basado en $R A M P S$.

Entrada: paquetes perdidos $L(i)$, paquetes enviados $S(i)$

Salida: Tiempo total para el periodo de backoff $(T)$

si Primera transmisión de la estación dentro de esta $\mathrm{AC}$ entonces

$$
\begin{aligned}
a_{A C}(i) & \leftarrow 0 \\
a_{A C}(i)_{a v g} & \leftarrow 0 \\
\text { i no } & \leftarrow \frac{L(i-1)-L(i)}{S(i-1)-S(i)} \\
a_{A C}(i) & \leftarrow(1-\alpha) \cdot a_{A C}(i)+\alpha \cdot a_{A C}(i-1)_{a v g} \\
a_{A C}(i)_{\text {avg }} & \leftarrow(1-\alpha)
\end{aligned}
$$

si no

fin si

$F_{\max }(i) \leftarrow[C W(i)+1]^{a_{A C}(i)_{a v g}}$

$F(i) \leftarrow \operatorname{rand}\left[0, F_{\max }(i)\right]$

$T \leftarrow \operatorname{rand}[1, C W(i)+1]+F(i)$

devolver $T$ 


\section{Capítulo 7 \\ El modelo EDCA: metodología de evaluación}

La derivación del modelo para EDCA como una extensión directa del modelo por cadenas de Markov propuesto por Bianchi para DCF en IEEE 802.11 [7] constituye un referente común en la evaluación del rendimiento para EDCA presentado por un significativo número de trabajos recientes en el área. Como parte de este trabajo relacionado podemos citar [22, 23, 24, 25] entre algunos de los más relevantes.

Para el propósito de nuestra investigación, una repercusión de esta tendencia reside en las consideraciones para el modelado de las fuentes generadoras de tráfico. Conforme a las características del modelo, el patrón para la distribución del tráfico debe tener en cuenta las siguientes consideraciones:

- Debido a la incertidumbre inherente en el proceso de arribo de los paquetes a la capa MAC, los cambios de estado en el proceso de Markov pueden ocurrir en instantes arbitrarios y el tiempo de permanencia del proceso en un estado no puede conocerse de antemano.

- Como el tiempo de permanencia en cada estado no está determinado, la distribución temporal de un proceso de Markov es exponencial. Dado que es la única distribución sin memoria en el tiempo, la duración del proceso en un estado no influye en el comportamiento futuro del sistema.

- Se asume que el proceso de colisión de paquetes sigue una distribución de Bernoulli (proceso sin memoria). Como se reporta en [7,26] esta suposición nos permite describir el estado de una estación bajo el modelo DCF (y por consecuencia su extensión para el modelo EDCA) mediante el modelo de cadenas de Markov.

Teniendo en cuenta los puntos anteriores, Consideremos un intervalo $K_{t}$ donde estamos interesados en representar el número de llegadas en dicho intervalo (arribo de paquetes a la capa MAC) mediante una variable aleatoria.

Dividiendo el intervalo $(0, t)$ en subintervalos de longitud $\Delta$ obtenemos $n$ de estos subintervalos, como se muestra en la Figura 7.1. Consideremos ahora que cuando $\Delta$ tiende a cero 


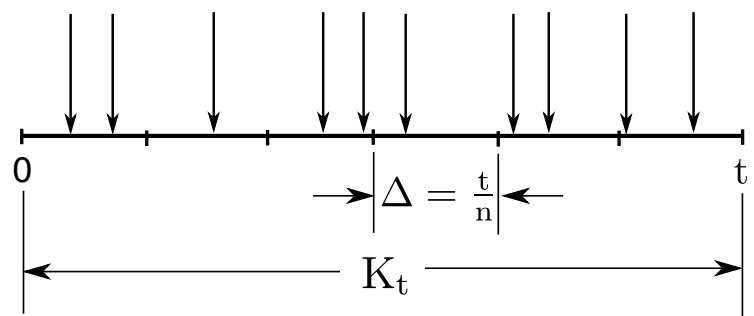

Figura 7.1: Proceso de arribo de paquetes a la capa MAC.

el número de arribos en cada subintervalo es a lo más de uno (muy probablemente uno o ninguno). Entonces, la probabilidad asociada al proceso se puede estudiar como una variable aleatoria que sigue una distribución binomial, una distribución de probabilidad discreta que mide el número de éxitos en una secuencia de $n$ ensayos de Bernoulli independientes entre sí. Tenemos entonces que la función de probabilidad asociada al proceso es

$$
P\left(K_{t}=k\right)=P_{k}=\left(\begin{array}{l}
n \\
k
\end{array}\right) p^{k}(1-p)^{n-k}
$$

Para una constante arbitraria $\lambda$ y con $\Delta \rightarrow 0$ (tal que se pueda asegurar $\lambda \Delta<1$ ), podemos considerar como la probabilidad de arribo al producto $\lambda \Delta$. Esto es

$$
\begin{aligned}
P\left(K_{t}=k\right)=P_{k} & =\left(\begin{array}{l}
n \\
k
\end{array}\right)(\lambda \Delta)^{k}(1-\lambda \Delta)^{n-k} \\
& =\frac{n !}{(n-k) ! k !}(\lambda \Delta)^{k}(1-\lambda \Delta)^{n-k} \\
& =\frac{n(n-1) \cdots(n-k+1)}{k !}\left(\frac{\lambda t}{n}\right)^{n}\left(1-\frac{\lambda t}{n}\right)^{-k} \\
& =\left(\frac{\lambda t}{k !}\right)^{k} \frac{n(n-1) \cdots(n-k+1)}{k !}\left(1-\frac{\lambda t}{n}\right)^{n}\left(1-\frac{\lambda t}{n}\right)^{-k}
\end{aligned}
$$

En donde se ha despreciado la contribución a la probabilidad de la ocurrencia de dos o más arribos por subintervalo. En el límite cuando $n \rightarrow \infty$, la función de probabilidad asociada al proceso resulta

$$
\begin{aligned}
& P_{k}=\lim _{n \rightarrow \infty}\left[\left(\frac{\lambda t}{k !}\right)^{k} \frac{n(n-1) \cdots(n-k+1)}{k !}\left(1-\frac{\lambda t}{n}\right)^{n}\left(1-\frac{\lambda t}{n}\right)^{-k}\right] \\
& P_{k}=\frac{(\lambda t)^{k}}{k !} e^{-\lambda t} \quad \lambda>0 ; k=0,1, \ldots
\end{aligned}
$$


El resultado al que se llega tomando en consideración los requerimientos del modelo EDCA y las características del proceso de arribo de paquetes a la capa MAC, nos conduce a una distribución de probabilidad bien definida y popular en la teoría de filas de espera, la distribución de Poisson ${ }^{1}$. En base a lo anterior, el modelado de las fuentes generadoras de tráfico como procesos de arribo de paquetes con distribución de Poisson ha sido una técnica empleada en el estudio y evaluación del rendimiento para el modelo DCF, como referencias directas para este enfoque podemos citar los trabajos [7, 26]. Debido a la estrecha relación entre el modelo DCF y el derivado para EDCA, este enfoque sigue siendo válido.

\subsection{Consideraciones para el modelo de tráfico en ED- $\mathrm{CA}$}

En la teoría de filas de espera, el proceso de arribo de Poisson ha sido el modelo de referencia para tráfico de voz y datos [28]. La suposición tradicional del modelo ha sido justificada al argumentar que la agregación de múltiples procesos de renovación ${ }^{2}$ independientes e idénticamente distribuidos (i.i.d.) tiende a un proceso de Poisson cuando su número se incrementa [29]. Los arribos del proceso de Poisson con tasa media $\lambda$ están separados por tiempos inter-arribo $\left\{\tau_{1}, \tau_{2}, \cdots\right\}$ que son i.i.d. de acuerdo a una distribución de probabilidad exponencial $p(\tau)=\lambda e^{-\lambda \tau}, \tau \geq 0$.

Para el caso del vídeo se adopta el enfoque mostrado en [30], donde el tráfico de vídeo es modelado por una fuente $\mathrm{ON} / \mathrm{OFF}$ con distribución exponencial. El estado de la fuente ON/OFF puede ser representado mediante una cadena de Markov de dos, estados continua en el tiempo, según se muestra en la Figura 7.2.

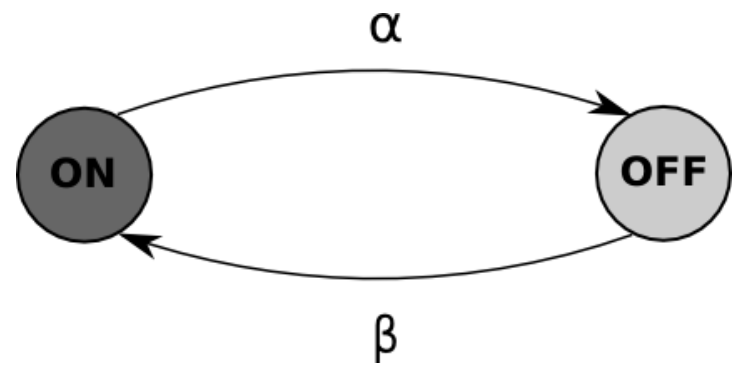

Figura 7.2: Cadena de Markov para la fuente ON/OFF

En el estado activo, los paquetes son generados como un proceso de Poisson con tasa $\lambda$, en consecuencia los tiempos inter-arribo de los paquetes están exponencialmente distribuidos con tasa $\tau$. En el estado inactivo, no se generan paquetes. Este modelo ON/OFF puede ser considerado como un simple ejemplo de un proceso de Markov modulado en tasa ${ }^{3}$ [28]

\footnotetext{
${ }^{1}$ Para una demostración formal del resultado aquí mostrado se sugiere al lector consultar [27].

${ }^{2}$ Para una deducción formal sobre la derivación de los procesos de Poisson y Bernoulli como procesos de renovación y su vínculo con las cadenas de Markov se sugiere al lector consultar [27].

${ }^{3}$ El trabajo de Fischer et al [31] constituye la referencia clásica en el tema.
} 
que en esencia es una combinación de 2 procesos. El proceso de Poisson de generación de paquetes constituye el proceso básico. Este proceso básico es multiplicado por un proceso de modulación que es la cadena de Markov mostrada en la Figura 7.2. El efecto del proceso de modulación es el cancelar o interrumpir el proceso de Poisson durante los períodos inactivos, es por ello que este tipo específico de proceso de modulación de Markov es denominado proceso interrumpido de Poisson. El patrón de tráfico generado a partir del modelo de fuente ON/OFF con una distribución temporal exponencial se muestra en la Figura 7.3 [30].

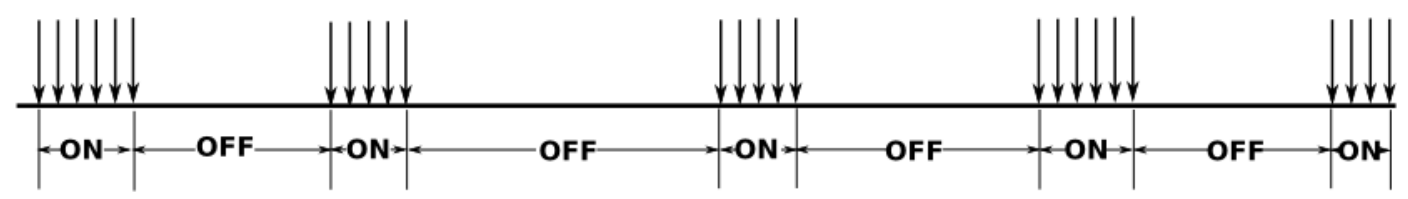

Figura 7.3: Patrón de tráfico generado a partir de la fuente ON/OFF exponencial.

\subsection{Métricas de desempeño para evaluar la calidad de servicio}

El concepto de calidad de servicio (o $Q o S$ ) aplicado al entorno de las redes de computadoras se refiere a: "las garantías que la red ofrece para satisfacer el desempeño predeterminado para un conjunto heterogéneo de servicios solicitados por el usuario". En otras palabras, podemos evaluar el desempeño de la calidad de servicio en base al análisis cualitativo de parámetros tales como: la tasa de bit efectiva, el retardo extremo a extremo (o latencia), variabilidad del retardo, pérdida de paquetes, etcétera. Es por ello que el diseño de mecanismos que nos permitan garantizar a los usuarios finales y a sus aplicaciones la provisión de calidad de servicio en la red es un objetivo de gran importancia.

Para el caso de las aplicaciones multimedia se consideran las siguientes características:

- Necesitan un ancho de banda mínimo

- Son muy sensibles al retardo de extremo a extremo

- Son sensibles a la variabilidad del retardo

- Relativamente tolerantes a pérdidas de paquetes (p. ej. en el caso del audio interactivo, tasas de pérdida de hasta un $5 \%$ se consideran adecuadas [32]).

Además, hay que integrar las aplicaciones multimedia con aplicaciones tradicionales que al ser poco sensibles al retardo extremo a extremo o a la variabilidad del retardo, son denominadas como elásticas. Esta diferenciación ,estableciendo prioridades basado en los requerimientos por tipo de aplicación, es el objetivo principal del algoritmo EDCA propuesto en el estándar [1]. 
Por tanto, la calidad de servicio se puede definir como el proceso de entrega de datos de una forma fiable, incluyendo aspectos importantes como son la pérdida de paquetes y el retardo. Estos deben ser mínimos o casi nulos, principalmente para aplicaciones en tiempo real, ya que los retardos y la pérdida de paquetes afecta en gran medida a estos servicios (e.g. VoIP, AVoD).

A continuación se enlistan las características de la serie de parámetros que definen la calidad de servicio:

\section{Pérdida de paquetes}

La pérdida de paquetes se produce cuando éstos no llegan a su destino final. Esto sucede por diversos motivos, por ejemplo, cuando se congestiona la red (los dispositivos de encaminamiento llenan de paquetes sus buffers y los desbordan) se pierden paquetes. Otro factor que puede provocar pérdida de paquetes son las interferencias en el medio inalámbrico, debido a la existencia de dispositivos que operan sobre la misma banda de frecuencias. El porcentaje de paquetes perdidos o descartados se puede obtener a partir de (7.4).

$$
P_{p p}=\frac{P_{p}}{P_{t}} \times 100 .
$$

Donde:

$P_{P P}=$ Porcentaje de paquetes perdidos.

$P_{p}=$ Paquetes perdidos.

$P_{t}=$ Paquetes transmitidos.

$$
P_{p}=P_{t}-P_{r} .
$$

Donde:

$P_{p}=$ Paquetes perdidos.

$P_{t}=$ Paquetes transmitidos.

$P_{r}=$ Paquetes recibidos.

\section{Retardo (latencia)}

El retardo de extremo a extremo o latencia, es el tiempo total que transcurre desde que un paquete de datos es generado en un nodo fuente hasta que es recibido por el nodo destino. Este parámetro se mide en unidades temporales. 


\section{Variabilidad del retardo (jitter)}

El jitter es la variación en el retardo que sufren los diferentes paquetes de pertenecientes a un mismo flujo de tráfico para alcanzar al nodo destino. Esto debido a que, aunque los paquetes enviados pueden seguir un mismo camino dentro de la red, se van a ver afectados por distintos factores (retardos en la fila de espera, variaciones repentinas en las condiciones del medio inalámbrico, espera por asignación de turnos, etcétera).

\section{Tasa de transferencia efectiva (throughput)}

La tasa de transferencia efectiva es la velocidad con la que es recibido el tráfico en un nodo destino. Se puede obtener a partir de la expresión (7.6).

$$
T_{E}=\frac{D_{r}}{t_{T}} .
$$

Donde:

$T_{E}=$ Tasa de bit efectiva.

$D_{r}=$ Datos recibidos (bits).

$t_{T}=$ Tiempo (en segundos) desde que la fuente inyecta el primer bit de un paquete en la red hasta que el destino recibe el último bit de ese paquete.

\section{Colisiones virtuales}

Una de las principales características en la función de acceso al medio EDCA es la introducción de un registro de almacenamiento exclusivo para cada una de las AC, que en su funcionamiento pueden considerarse como ciclos DCF virtuales. Cuando dos o más de estos ciclos virtuales intentan transmitir al mismo tiempo se dice que ocurre una colisión virtual. Las colisiones virtuales detectadas durante la etapa de asignación del turno de transmisión entre los flujos de tráfico al interior de la estación son un indicador de la capacidad del algoritmo EDCA para ofrecer un servicio diferenciado de calidad de servicio.

\section{Paquetes en colisión durante su transmisión por el canal inalámbrico}

El estándar [1] define a CSMA/CA como protocolo de acceso múltiple al medio compartido. Debido a que CSMA/CA busca minimizar la probabilidad de ocurrencia de que dos o más estaciones transmitan simultáneamente, la cantidad de paquetes detectados en colisión durante su transmisión por el canal inalámbrico sirve como métrica respecto a la capacidad del algoritmo EDCA para gestionar el acceso local al medio inalámbrico. Al disminuir el numero de paquetes en colisión disminuye la utilización del canal en retransmisiones y aumenta la tasa de transmisión efectiva. 


\section{Capítulo 8 Simulaciones y resultados}

En los apartados anteriores revisamos los conceptos teóricos relevantes que comprende el proyecto: los estándares IEEE 802.11 e IEEE 802.11e, el estado del arte y la descripción del algoritmo ACI-EDCA a implementar.

A continuación, analizamos el algoritmo ACI-EDCA utilizando la herramienta NS-2. Debido a que el estándar IEEE 802.11e no está actualmente soportado por el simulador, empleamos la extensión propuesta por Casetti [5] que permite utilizar el modo EDCA/HCCA en NS-2 ${ }^{1}$. Tras su instalación, implementamos nuestra propuesta modificando el código del algoritmo de referencia para IEEE 802.11e.

En este capítulo describimos el conjunto de características particulares de los escenarios de simulación empleados, las consideraciones en el modelado de las fuentes generadoras de tráfico, las medidas de desempeño a evaluar y el análisis de los resultados obtenidos.

\subsection{Fuentes de tráfico}

Se consideran dos tipos de tráfico con requisitos de QoS: VoIP y vídeo a la demanda (VoD). Además, se utiliza el mecanismo de diferenciación de EDCA para transmitir concurrentemente tráfico que no posee requisitos específicos de QoS .

\section{Fuente generadora de vídeo}

Para las fuentes generadoras de vídeo, una consideración derivada directamente del análisis estadístico en [30] es la relación que guardan el parámetro para la distribución exponencial $(\lambda))$ para los periodos en que el tráfico alterna entre los estados on y off:

$$
\frac{\lambda_{(\text {periodo }- \text { on })}}{\lambda_{(\text {periodo }- \text { off })}} \sim[0,399,0,547]
$$

De acuerdo a las consideraciones establecidas para el modelo, se tiene que en promedio la proporción para la permanencia entre estados está contenida dentro del intervalo. Una

\footnotetext{
${ }^{1}$ El apéndice $A$ presenta las características más relevantes para el modelo inalámbrico de IEEE 802.11 en NS-2
} 
consecuencia directa de este resultado es que una vez definido el parámetro de la distribución exponencial para el periodo on u off, el conjunto de valores para la característica exponencial del otro periodo está acotado. Por simplicidad, elegimos fijar el valor para la intensidad de tráfico $^{2}$ en 0.5 y determinamos el parámetro para la distribución exponencial del periodo on conforme a las características del estándar para compresión de vídeo H.264/MPEG-4 AVC 3 .

\section{Tráfico VoIP}

El tráfico de voz es generado por una fuente de Poisson. La característica para el tráfico considerada es la requerida para el codec G.729 [33], con tasa de bit de 8 Kbps y consumo de ancho de banda de $11.2 \mathrm{Kbps}$ por llamada.

\section{Tráfico Best-Effort}

El tráfico Best-Effort es considerado como un flujo de datos a una tasa constante de 1 Mbps [21].

\section{Tráfico de Background}

La característica para el tráfico de background es generada mediante una fuente de Poisson a una tasa de $400 \mathrm{kbps}$ [34].

En la Tabla 8.1 se resumen los parámetros de simulación empleados para EDCA.

\begin{tabular}{ccccc}
\hline & Voz & Video & Best-Effort & Background \\
\hline Modelo de tráfico & Poisson & IPP & CBR & Poisson \\
Protocolo de transporte & UDP & UDP & TCP & UDP \\
AC & VO & VI & BE & BG \\
CWmin & 7 & 15 & 15 & 31 \\
CWmax & 15 & 31 & 1023 & 1023 \\
AIFS & $30 \mu \mathrm{s}$ & $50 \mu \mathrm{s}$ & $70 \mu \mathrm{s}$ & $150 \mu \mathrm{s}$ \\
Tamaño de paquete & 250 & 510 & 250 & 250 \\
Tasa de envío & $11.2 \mathrm{kbps}$ & $1512 \mathrm{kbps}$ & $1024 \mathrm{kbps}$ & $400 \mathrm{kbps}$ \\
\hline
\end{tabular}

Tabla 8.1: Parámetros de simulación para EDCA.

\footnotetext{
${ }^{2}$ Así se define a la relación de permanencia entre estados durante la derivación formal de la solución general para un proceso de muerte y nacimiento.

${ }^{3}$ Producto del esfuerzo conjunto del grupo Joint Video Team (JVT). El estándar UIT-T H.264 y la norma ISO/IEC MPEG-4 AVC (formalmente, norma ISO/IEC 14496-10 - MPEG-4 Parte 10, "Advanced Video Coding"). Es actualmente uno de los formatos más utilizados para el registro, la compresión y la distribución de vídeo de alta definición.
} 


\subsection{Parámetros de simulación}

Una vez descritas las fuentes generadoras de tráfico, el siguiente paso consiste en describir las características generales de la simulación. Para generar las estadísticas de desempeño, se han realizado 40 simulaciones de 1000 segundos para cada una de las configuraciones de la topología de la red. Cada una de las estaciones dentro de la topología de red "despierta" sobre la circunferencia de un círculo con un radio de 25 metros en cuyo centro se localiza el punto de acceso. Para introducir variaciones en la topología de red modificamos el número de estaciones activas dentro de la WLAN hasta un máximo de 30, incorporando 5 estaciones adicionales cada vez que una simulación concluye y regresando a un tamaño mínimo de 5 estaciones una vez concluida la simulación con 30 estaciones activas. Cuando una estación "despierta" al inicio de la simulación intentará establecer un par de llamadas de VoIP bidireccionales, solicitará un flujo descendente de video-streaming y establecerá una comunicación bidireccional en cuanto al tráfico de background y best-effort. Cada una de las fuentes generadoras de tráfico inicia su periodo activo de transmisión de forma aleatoria e independiente de las otras fuentes de tráfico. En la Figura 8.1 se muestra un ejemplo de la configuración de red empleada en la simulación.

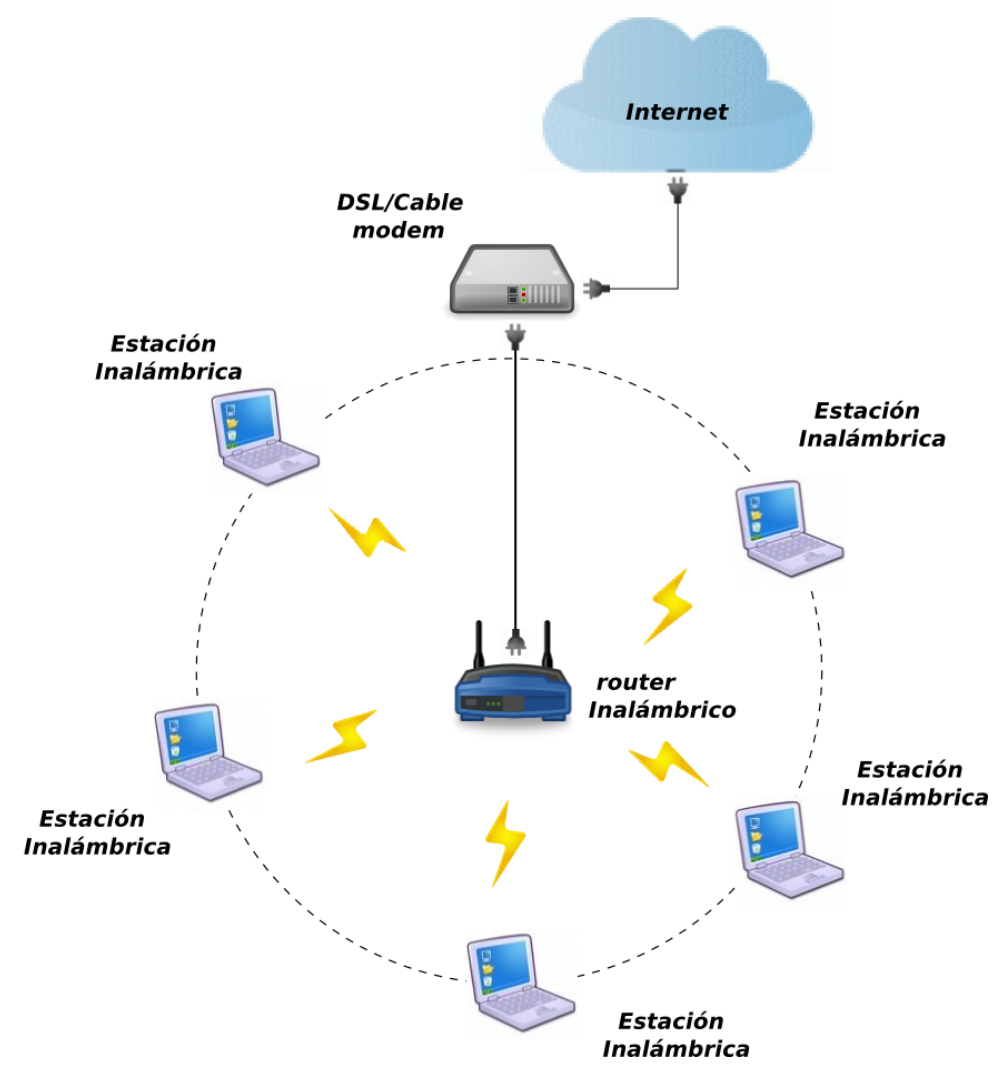

Figura 8.1: Topología de la red a simular. 
En la Tabla 8.2 se resumen los parámetros más relevantes a considerar de la capa MAC y de la capa física.

\begin{tabular}{cc}
\hline Parámetro & Valor \\
\hline SIFS & $10 \mu \mathrm{s}$ \\
PIFS & $30 \mu \mathrm{s}$ \\
DIFS & $50 \mu \mathrm{s}$ \\
Tiempo de ranura & $20 \mu \mathrm{s}$ \\
Tasa de datos & $11 \mathrm{Mb} / \mathrm{s}$ \\
Tasa mínima & $1 \mathrm{Mb} / \mathrm{s}$ \\
\hline
\end{tabular}

Tabla 8.2: Parámetros de la capa MAC y de la capa física.

\subsection{Resultados}

Las Figuras 8.3 y 8.2, muestran la comparativa de desempeño entre los tres algoritmos respecto a la tasa de colisiones durante el acceso al canal y las colisiones virtuales detectadas durante la asignación de la TXOP, respectivamente. Los resultados obtenidos exhiben una evidente mejora respecto al desempeño de los algoritmos de referencia $[2,3]$, en cuanto a la capacidad del algoritmo para mantener una baja tasa de colisiones. Este resultado tiene como consecuencia directa el aumento en la tasa de transmisión efectiva al disminuir el numero de retransmisiones por paquetes en colisión (Figura 8.3) así como en el retardo en el acceso al canal debido al mecanismo de autogestión en la estación para proporcionar un servicio diferenciado a los flujos de tráfico por AC al interior de la misma (Figura 8.2).

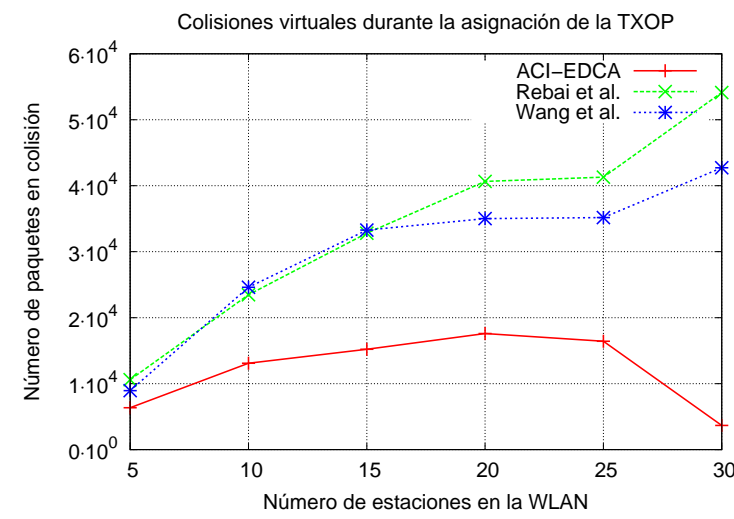

Figura 8.2: Colisiones virtuales detectadas durante la asignación del turno de transmisión.

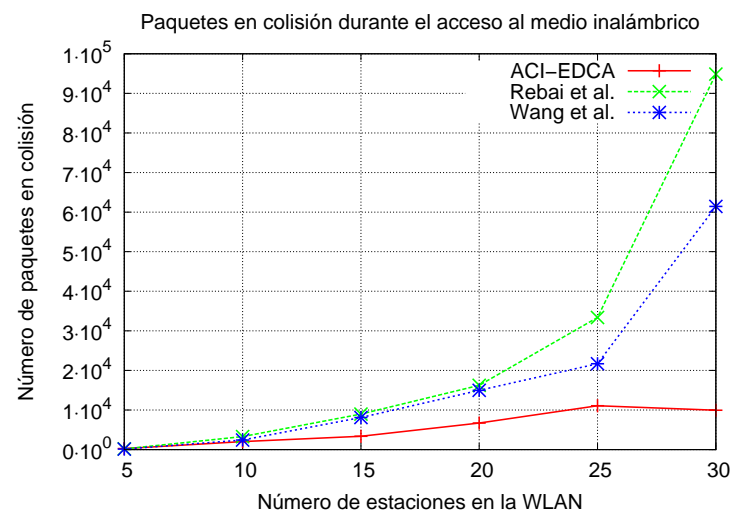

Figura 8.3: Paquetes en colisión durante su transmisión por el canal inalámbrico.

En condiciones de alta demanda sobre los recursos del sistema, la estrategia del algoritmo EDCA esta comprometida a la asignación de las oportunidades de transmisión a las categorías 
de tráfico de alta prioridad. Debido a que los tres algoritmos evaluados, en esencia, conservan el estricto sistema de prioridades del algoritmo EDCA original, el comportamiento esperado para las categorías de baja prioridad es la disminución de la tasa de transmisión efectiva al aumentar el número de estaciones. Como puede verse en la comparativa de desempeño para el tráfico servido por la categoría más baja de tráfico (Figura 8.4.a) el algoritmo propuesto en este trabajo consigue un aumento en la tasa de transmisión. Por otra parte, respecto al tráfico best-effort (Figura 8.4.b) el algoritmo propuesto es más restrictivo y en consecuencia la tasa de transmisión para esta categoría tiende a disminuir. Finalmente, respecto a las categorías de vídeo (Figura 8.4.c) y de voz (Figura 8.4.d), la comparativa de desempeño muestra que el algoritmo propuesto ofrece una mejor solución para transmisión de tráfico multimedia.

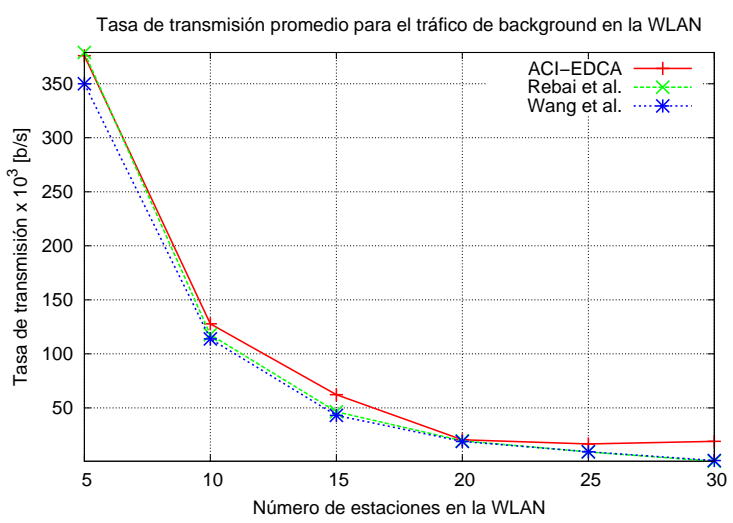

(a)

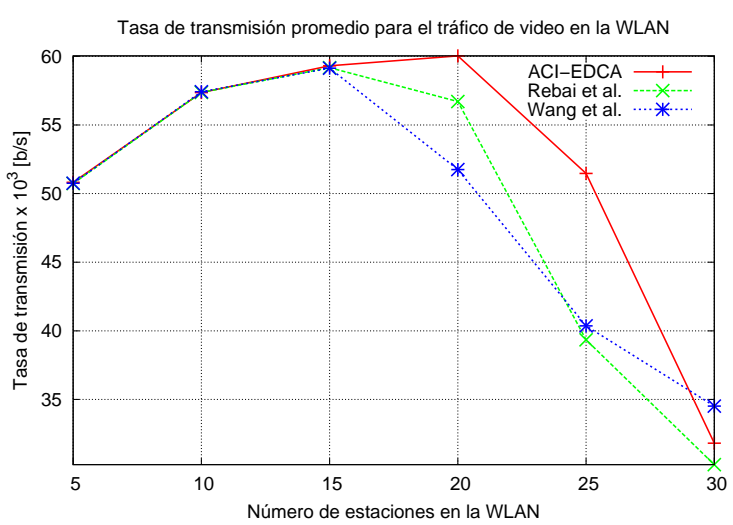

(c)

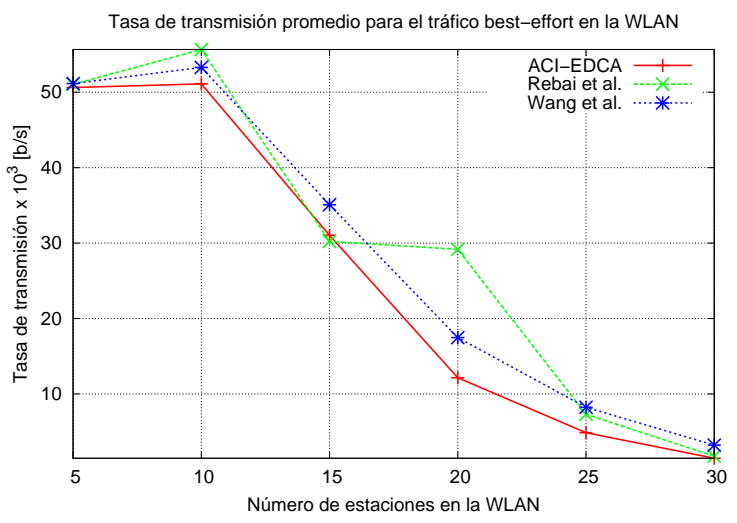

(b)

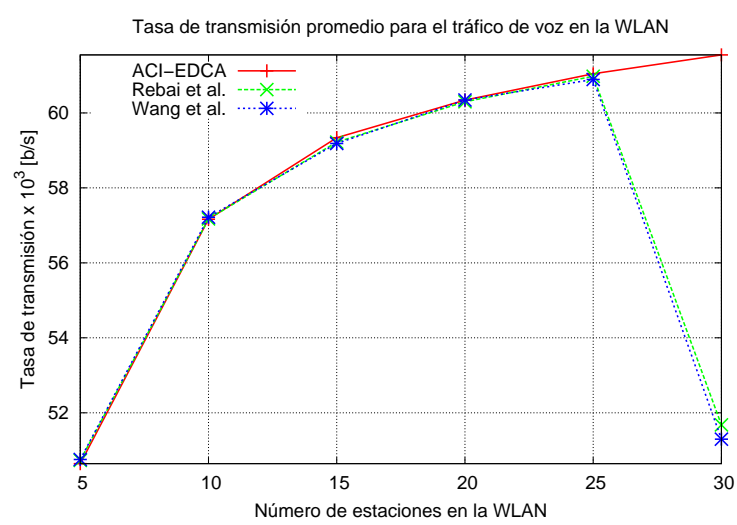

(d)

Figura 8.4: Comparación de desempeño para el throughput por AC.

En cuanto a la evaluación para el retardo de extremo a extremo. Uno de los resultados más relevantes obtenidos mediante nuestro algoritmo es la anulación de los picos en el retardo observado en la comparativa de desempeño para el trabajo de referencia. De esta forma, al 
variar la carga de tráfico sobre la red el incremento en el período de contención será gradual. Se observa que para la categoría de tráfico best-effort (Figura 8.5.b) RAMPS-EDCA [2] obtiene un mejor desempeño. Por otra parte, para la categoría de tráfico de más alta prioridad (Figura 8.5.d) el algoritmo propuesto consigue una cota menor para la característica del retardo, mientras mantiene un desempeño semejante al obtenido por RAMPS-EDCA para la categoría de vídeo (Figura 8.5.c).

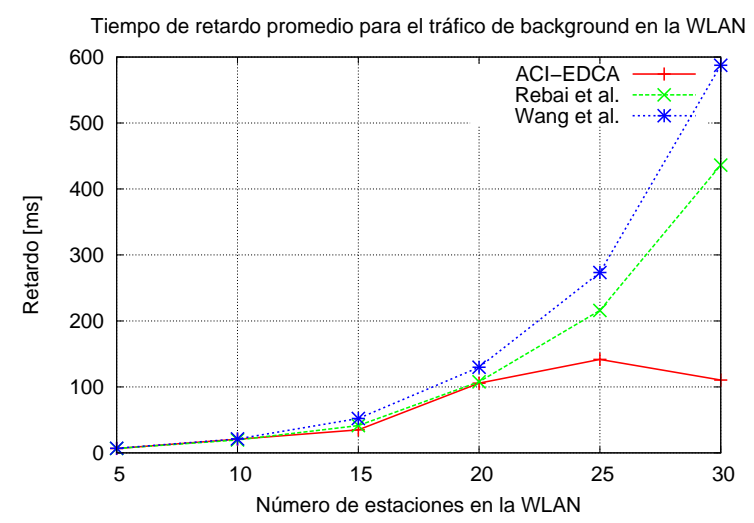

(a)

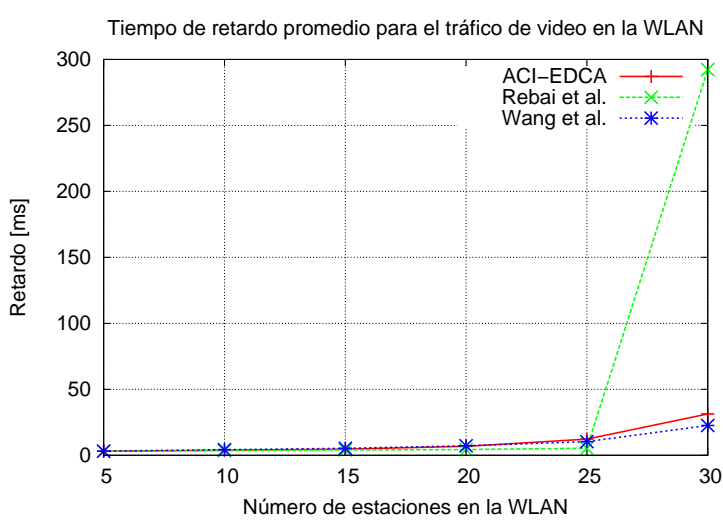

(c)

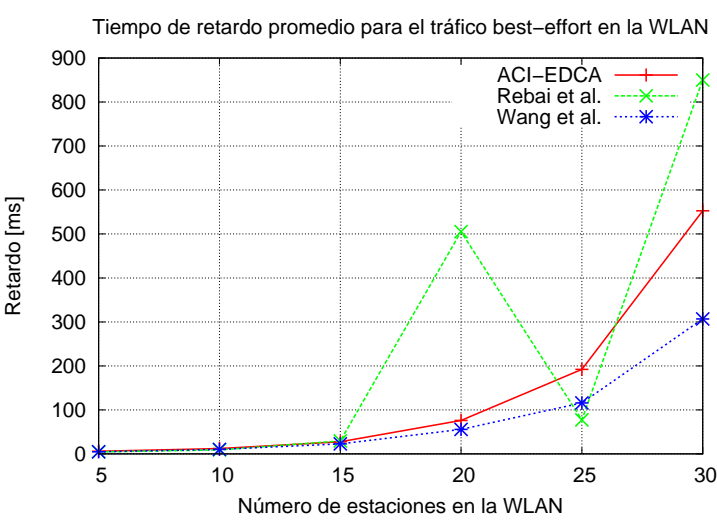

(b)

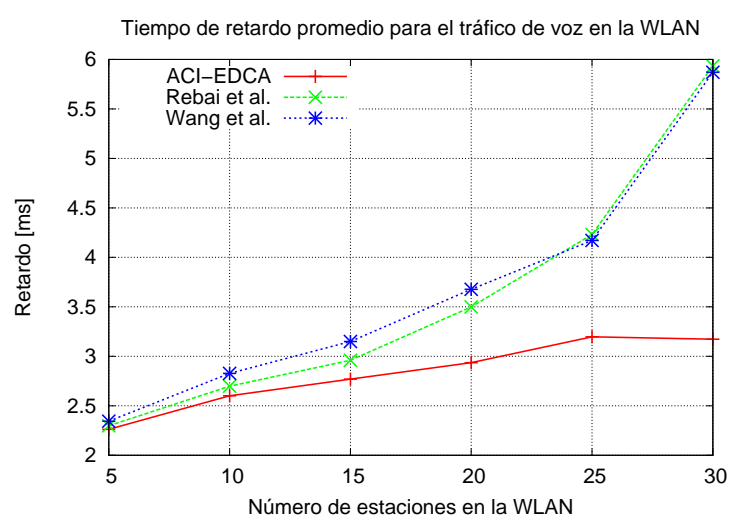

(d)

Figura 8.5: Comparación de desempeño para el retardo por AC.

Respecto a la comparativa de desempeño para la varianza, un resultado destacable en nuestro algoritmo es la homologación en el tiempo de transmisión para los flujos de tráfico de audio (Figura 8.6.d) y vídeo (Figura 8.6.c). Estas dos categorías son especialmente sensibles a las fluctuaciones en los tiempos de transmisión, por lo que establecer un control estricto sobre este parámetro contribuye a garantizar que los flujos de tráfico multimedia alcancen los requisitos de QoS requeridos. Respecto al tráfico servido por las categorías de menor prioridad, nuestra propuesta tiende a causar una sensible disminución en la variabilidad del retardo respecto al trabajo de referencia. 


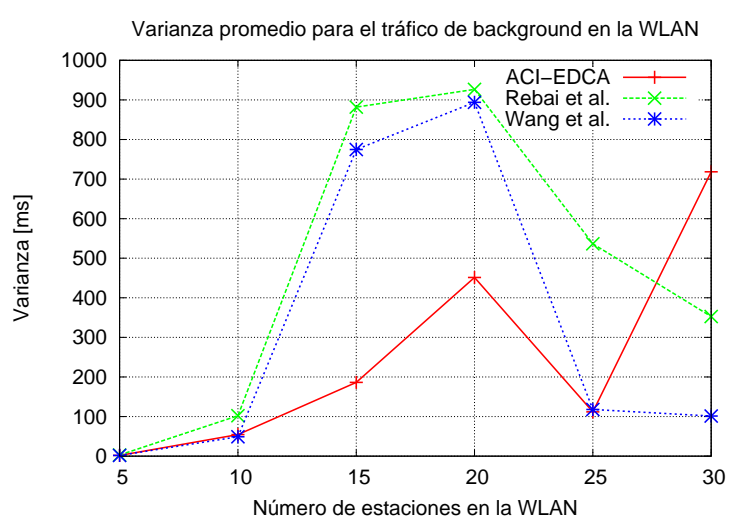

(a)

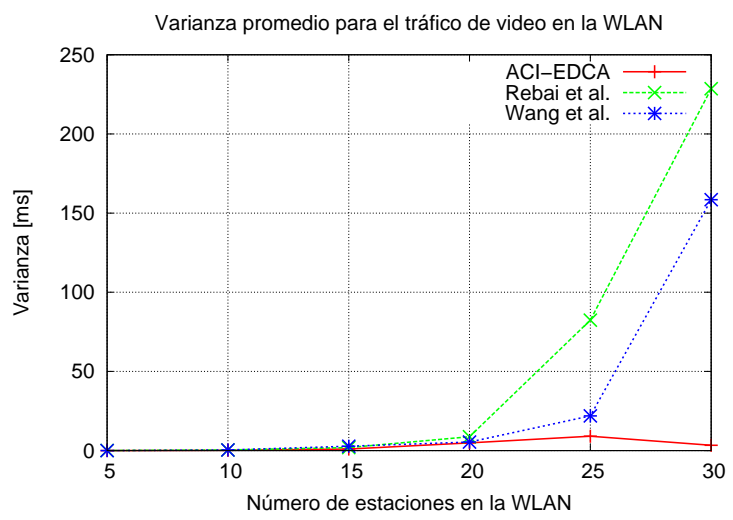

(c)

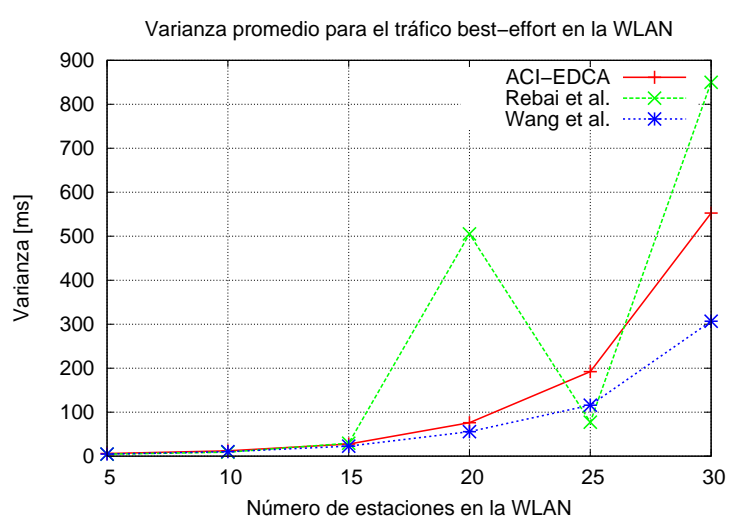

(b)

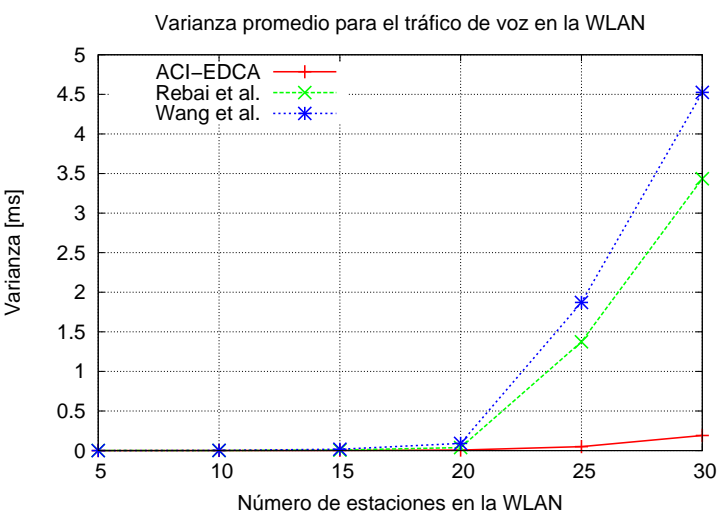

(d)

Figura 8.6: Comparación de desempeño para la varianza por AC. 


\section{Capítulo 9 Conclusiones y perspectivas}

En este trabajo hemos descrito un algoritmo EDCA que proporciona diferenciación interAC mientras ajusta la tasa de transmisión por AC acorde a las condiciones actuales del medio de transmisión. Para evaluar el desempeño de nuestro algoritmo, lo comparamos con esquemas similares ya existentes.

Para lo anterior, se presentó en primer lugar una revisión de las funciones de acceso al medio para el estándar IEEE 802.11 e IEEE 802.11e. De esta revisión se desprende que las colisiones en el acceso al medio, el periodo de contención en la asignación de la TXOP, la tasa de pérdida, el retardo y la variabilidad en el retardo son los fenómenos que más impactan la QoS en las aplicaciones multimedia.

Un resultado importante observado en el desempeño del algoritmo ACI-EDCA, es la sensible disminución en la tasa de colisión respecto a CI-EDCA y RAMPS-EDCA, lo que se refleja en un aumento en la tasa efectiva de transmisión por AC. Como resultado directo de la disminución en la tasa de colisiones virtuales durante la asignación de la TXOP, podemos concluir que la estrategia en el algoritmo ACI-EDCA consigue un mejor desempeño respecto a la diferenciación al servicio ofrecido por AC.

Como resultado adicional, los resultados obtenidos muestran que la estrategia de EDCA es más sensible a la adaptación a las condiciones del medio mediante el parámetro de backoff, que al efecto del ajuste dinámico sobre AIFS. Esto resulta evidente al observar que el desempeño de RAMPS [2] tiende a emular el comportamiento de ACI-EDCA. No obstante, el esquema de clasificación por actividad de la estación basado en AIFS contribuye a la estrategia adaptativa en limitar el número de estaciones por AC transmitiendo concurrentemente con el mismo conjunto de parámetros MAC, lo que se ve reflejado en la disminución de la tasa de colisiones durante el acceso al medio.

Nuestra propuesta estima la capacidad del canal para ofrecer un servicio diferenciado de tráfico con distintos requerimientos de QoS mediante un algoritmo de dos fases basado en la tasa de pérdida de la estación y la actividad por tipo de tráfico al interior de la misma, respectivamente.

Nuestro trabajo futuro se enfocará en adaptar el algoritmo ACI-EDCA en nuevos entornos de red no orientados a infraestructura (p. ej. redes mesh o MANETs), donde DCF es el modo de operación predominante en el acceso al medio. Otras direcciones de investigación en la que estamos interesados es evaluar el desempeño del algoritmo en el modo de operación ACIEDCA+HCCA, así como en escenarios donde el medio sea compartido con estaciones bajo el modo estándar de operación para IEEE 802.11 (DCF y DCF+PCF). 


\section{Referencias}

[1] Institute of Electrical and Electronics Engineers. Standard for Information Technology - Telecommunications and information exchange between systems - Local and metropolitan area networks - Specific requirements.Part 11: Wireless LAN Medium Access Control (MAC) and Physical Layer (PHY) Specifications. IEEE Standards Association, 1(12):1-1168, June 2007.

[2] WANG Jian-xin, S MAKFILE, and LI Jing. A random adaptive method to adjust MAC parameters in IEEE 802.11e WLANs. Journal of Central South University of Technology, 16(4):629-634, January 2009.

[3] A. R. Rebai, S. Hanafi, and H Alnuweiri. A new inter-node priority access enhancement scheme for IEEE_802.11 WLANs. International Conference on Intelligent Transport Systems Telecommunications, 2009. ITST'09, pages 395-405, October 2009.

[4] The Network Simulator version 2 NS-2. http://www.isi.edu/nsnam/ns/.

[5] Claudio Casetti. 802.11e implementation for the NS-2 simulator. Technical report, University of Pisa, Italy, 2005.

[6] Institute of Electrical and Electronics Engineers. Standard for Information Technology - Telecommunications and information exchange between systems - Local and metropolitan area networks - Specific requirements.Part 11: Wireless LAN Medium Access Control (MAC) and Physical Layer (PHY) Specifications. IEEE Computer Society, 1(1):1-528, June 1999.

[7] Giuseppe Bianchi. Performance analysis of the IEEE_802.11 Distributed Coordination Function. IEEE Journal on Selected Areas in Communications, 18(3):535-547, March 2000.

[8] Rob Flickenger. Redes Inalámbricas en los Países en Desarrollo. Canada's International Development Research Centre, Canada, 2009.

[9] J.K. Choi, J.S. Park, J.H. Lee, and K.S. Ryu. Review on QoS issues in IEEE_802.11 W-LAN. In Advanced Communication Technology, 2006. ICACT_2006. The 8th International Conference, volume 3, pages 2109-2113, February 2006. 
[10] W. Xing-feng, and L. Yuan-an. A Survey of WLAN QoS Systems Based on IEEE 802.11. International Journal of Computer Science and Network Security, 200\%. IJCSNS '07, 7(3):309-312, March 2007.

[11] Emilio Rafael Olvera Ochoa and Víctor M. Ramos R. Calidad de servicio en IEEE_802.11: situación actual y perspectivas. Memorias del VIII Congreso Internacional Sobre Innovación y Desarrollo Tecnológico, 2010. CIINDET'10, 1(1):741-747, November 2010.

[12] A. Kasentini, M. Naimi, A. Nafaa, and M. gueroui gueroui gueroui gueroui. Adaptive service differentiation for QoS provisioning in IEEE_802.11 wireless Ad Hoc network. In Proceedings of the 1st ACM international workshop on Performance evaluation of wireless ad hoc, sensor, and ubiquitous networks, volume ACM press of PE-WASUN '04, pages 39-45, New York, NY, USA, 2004. ACM.

[13] Der-Jiunn Deng and Hsu-Chun Yen. Quality-of-service provisioning system for multimedia transmission in IEEE_802.11 wireless LANs. Selected Areas in Communications, 23(6):1240-1252, June 2005.

[14] Y. Xiao, H. Li, K. Wu, K. Leung, and Qiang Ni. Reservation and grouping stations for the IEEE 802.11 DCF. NETWORKING'05 Proceedings of the 4th IFIP-TC6 international conference on Networking Technologies, Services, and Protocols; Performance of Computer and Communication Networks; Mobile and Wireless Communication Systems, 4:395-405, May 2005.

[15] Lei Guang, C.M. Assi, and A. Benslimane. Enhancing IEEE_802.11 random backoff in selfish environments. IEEE Transactions on Vehicular Technology, 57(3):1806-1822, May 2008.

[16] H. Zen, D. Habibi, J. Wyatt, and I. Ahmad. Converging voice, video and data in WLAN with QoS support. Wireless and Optical Communications Networks, 2008. WOCN '08., 3(5):1-5, May 2008.

[17] J. Wyatt, D. Habibi, I. Ahmad, and H. Zen. Providing QoS for symmetrical voice/video traffic in Wireless Networks. IEEE International Conference on Networks, 2007.ICON '07, 1(15):312-317, November 2007.

[18] Imad Aad, Qiang Ni, and Thierry Turletti. Enhancing IEEE_802.11 MAC in congested environments. Workshop on Applications and Services in Wireless Networks, 2004. $A S W N$ '04, 28(14):82-91, August 2004.

[19] Ouldooz Baghban Karimi and Mahmood Fathy. Adaptive end-to-end QoS for multimedia over heterogeneous wireless networks. Computers \&\& Electrical Engineering, 36(1):45-55, January 2010.

[20] Jung-Shian Li, Hung-Cheng Kao, and Wu-Han Lin. Achieving maximal VoIP calls in 802.11 wireless networks. Computer Communications, 33(11):1296-1303, January 2010. 
[21] Qiang Ni. Performance analysis and enhancements for IEEE_802.11e wireless networks. IEEE Network, 19(4):21-27, July-August 2005.

[22] Olav Østerbø and Paal E. Engelstad. WLAN QoS analysis - A new modelling approach for 802.11e. Technical Report 28, Telenor, November 2008.

[23] W. Zhang, J. Sun, J Liu, and H. Zhang . Performance analysis of IEEE 802.11e EDCA in wireless LANs. Journal of Zhejiang University SCIENCE A, 8(1):18-23, January 2007.

[24] W. Dapeng, Z. Yan, W. Muqing, and Z. Xiaojing. Medium access control access delay analysis of IEEE_802.11e wireless LAN. IET Communications, 3(6):1061-1070, 2009.

[25] Fei Peng, Hussein Alnuweiri, and Victor Leung. Theoretical performance evaluation of EDCA in IEEE_802.11e wireless LANs. European Transactions on Telecommunications 2010, 21(5):265-275, August 2010.

[26] Gion Reto Cantieni, Qiang Ni, Chadi Barakat, and Thierry Turletti. Performance analysis under finite load and improvements for multirate 802.11. Computer Comunications, 28(10):1095-1109, January 2005.

[27] Roy D. Yates and David Goodman. Probability and Stochastic Processes: A Friendly Introduction for Electrical and Computer Engineers. Jhon Wiley \& Sons Inc., 2nd. edition, May 2004.

[28] Hossein Bidgoli et al. The Handbook of Computer Networks, 3 Volume Set. Volume III, Part 2: Network Planning, Control, and Management. "Network Traffic Modeling"(Thomas M. Chen). Jhon Wiley \& Sons Inc., 2007.

[29] K. Sriram, and W. Whitt. Characterizing Superposition Arrival Processes in Packet Multiplexers for Voice and Data. IEEE Journal on Selected Areas in Communications, 4(6):833-846, September 1986.

[30] C. V. Seshaiah and K. Senbagam. Mathematical Modelling and Bandwidth Allocation for Video Teleconference Service Traffic. European Journal of Scientific Research, 60(4):619-629, September 2011.

[31] Wolfgang Fischer and Kathleen Meier-Hellstern. The Markov-modulated Poisson process (MMPP) cookbook. Performance Evaluation, 18(2):149-171, January 1993.

[32] Víctor Manuel Ramos Ramos. Robust and reliable multimedia transmission over the Internet. PhD thesis, Université de Nice, Sophia-Antipolis, France, 2004.

[33] Cisco. Voice over IP - Per Call Bandwidth Consumption. Document ID: 7934, February 2006.

[34] Ilias Politis, Michail Tsagkaropoulos, Tasos Dagiuklas, and Stavros Kotsopoulos. Study of the QoS of video traffic over integrated 3G-WLAN systems. In Proceedings of the 2nd international conference on Mobile multimedia communications, MobiMedia '06, pages 18-20, New York, NY, USA, September 2006. ACM. 
[35] Ke Liu. Understanding the implementation of IEEE MAC 802.11 standard in NS-2. The VINT Project: A Collaboration between Researchers at U.C. Berkeley, LBL, USC/ISI, and Xerox PARC, December 2007.

[36] G. Lei and C.M. Assi. Enhancing IEEE_802.11 random backoff in selfish environments. IEEE Transactions on Vehicular Technology, 57(3):1806-1822, May 2009.

[37] Qiang Ni. A survey of QoS enhancements for IEEE_802.11 wireless LAN. Wireless Communications and Mobile Computing, 4(3):547-566, August 2004.

[38] E. Lochin, L. Dairiane, and G. Jourjon. Guaranteed TCP Friendly Rate Control (gTFRC) for DiffServ/AF Network. Internet Draft draft-lochin-ietf-tsvwg-gtfrc-02, IETF , August, 2006.

[39] S. Floyd, M. Handley, J. Padhye, and J. Widmer. TCP Friendly Rate Control (TFRC). RFC 5348, Internet Engineering Task Force, September 2008. 


\section{Apéndice $\mathrm{A}$ \\ El simulador NS-2}

En el desarrollo del proyecto se ha utilizado el simulador NS-2, concretamente la versión 2.1b9a. NS-2 es un simulador de redes de eventos discretos y orientado a objetos, desarrollado por la Universidad de Berkeley en California y escrito en lenguaje C++ y OTcl. NS-2 se puede utilizar para simular tanto redes de área local (LAN) como redes de área amplia (WAN). Incluye modelos de simulación para tráfico basado en TCP, UDP y de protocolos de aplicación como FTP o Telnet. Además, soporta simulaciones basadas en patrones probabilísticos de tráfico (Exponencial, Pareto, etć) y simulaciones guiadas por archivos de trazas obtenidas a partir de la mediciones de las características de flujos de tráfico real. También incluye mecanismos de gestión de filas de espera y algoritmos de encaminamiento. Implementa algunos de los protocolos de la capa MAC para la simulación de redes LAN o WLAN.

En este apartado se resumen las principales características del simulador NS-2. Particularmente aquellas orientadas al modelo inalámbrico para IEEE 802.11 y la extensión propuesta por Casetti [5] que permite utilizar el modo EDCA/HCCA en NS-2.

\section{A.1. Características generales}

NS-2 esta escrito en $\mathrm{C}++$ y OTcl, con un intérprete OTcl que interactúa con el usuario. El simulador se apoya en una jerarquía de clases en $\mathrm{C}++$ (también llamada jerarquía compilada), y una jerarquía de clases OTcl con una estructura similar a la anterior (llamada jerarquía interpretada), estando ambas jerarquías relacionadas entre sí. Desde la perspectiva de un usuario, hay una correspondencia entre una clase en la jerarquía interpretada y una en la compilada (Figura A.1). La raíz de toda esta jerarquía se encuentra en la clase TclObject. Los usuarios crean nuevos objetos para la simulación a través del intérprete; estos objetos son instanciados dentro de OTcl, y son generados simétricamente por su objeto correspondiente en la jerarquía compilada. La jerarquía de clase interpretada se establece automáticamente através de métodos definidos en la clase TclObject.

La razón por la que NS-2 usa dos lenguajes diferentes se debe a que es necesario atender dos aspectos de simulación distintos. Por un lado, los detalles de la implementación requieren de un lenguaje de programación que pueda manejar con efectividad bytes, cabeceras de paquetes e implementar algoritmos que puedan tratar con efectividad grandes cantidades de datos. Para poder realizar esta tarea, la velocidad de ejecución es importante, pero no así el tiempo empleado en configuración. Por otro lado, una gran parte de la simulación en NS-2 


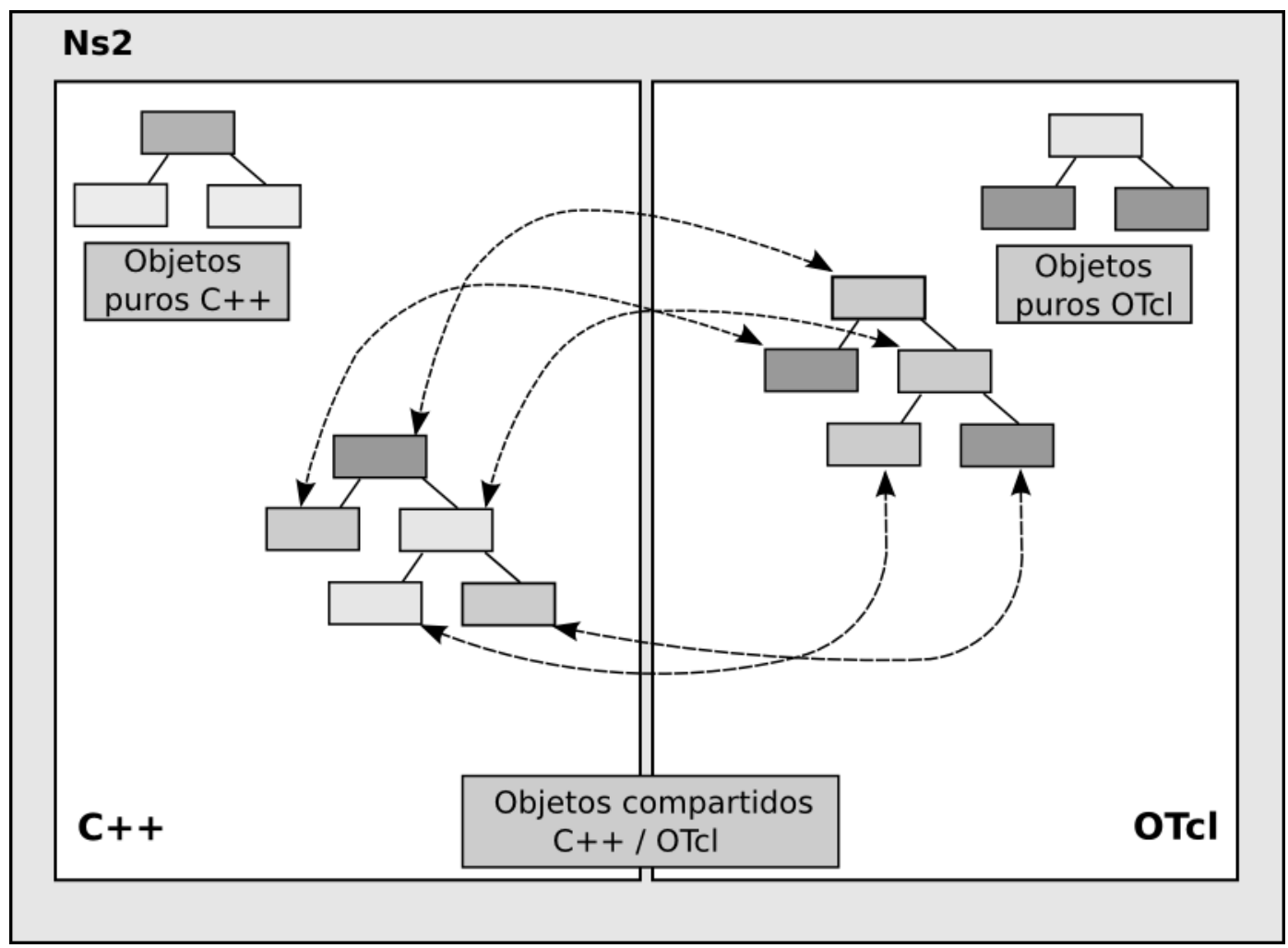

Figura A.1: Dualidad entre clases.

implica variar algunos parámetros de configuración para poder analizar distintos escenarios. En este caso, el tiempo empleado en la configuración por el usuario es más importante. Sin embargo, ya que la ejecución se produce una sola vez (al comienzo de la simulación), el tiempo de ejecución de esta parte es menos relevante. $\mathrm{C}++$ es un lenguaje de programación rápido en ejecución, haciéndolo ideal para detalles de implementación de protocolos. OTcl es más lento en ejecución pero se puede cambiar rápidamente y de forma interactiva, haciéndolo ideal para la configuración de la simulación.

Cuando una simulación termina, NS-2 crea uno o más archivos de texto de salida que contienen información detallada de los datos de simulación. Estos archivos pueden ser usados para analizar la simulación o como entrada de un simulador gráfico incorporado en NS-2 llamado animador de redes (NAM). NAM tiene una interfaz de usuario en la que se puede presentar gráficamente información como la topología de red (los nodos y sus enlaces) o el envío y descarte de paquetes (véase la Figura A.2).

\section{A.1.1. WLAN en NS-2}

El propósito de esta sección es presentar los elementos de interés sobre los que se basa el modelo inalámbrico de NS-2 para la simulación de redes inalámbricas. La descripción a detalle sobre como la interacción entre las clases componentes de la estructura MobileNode 


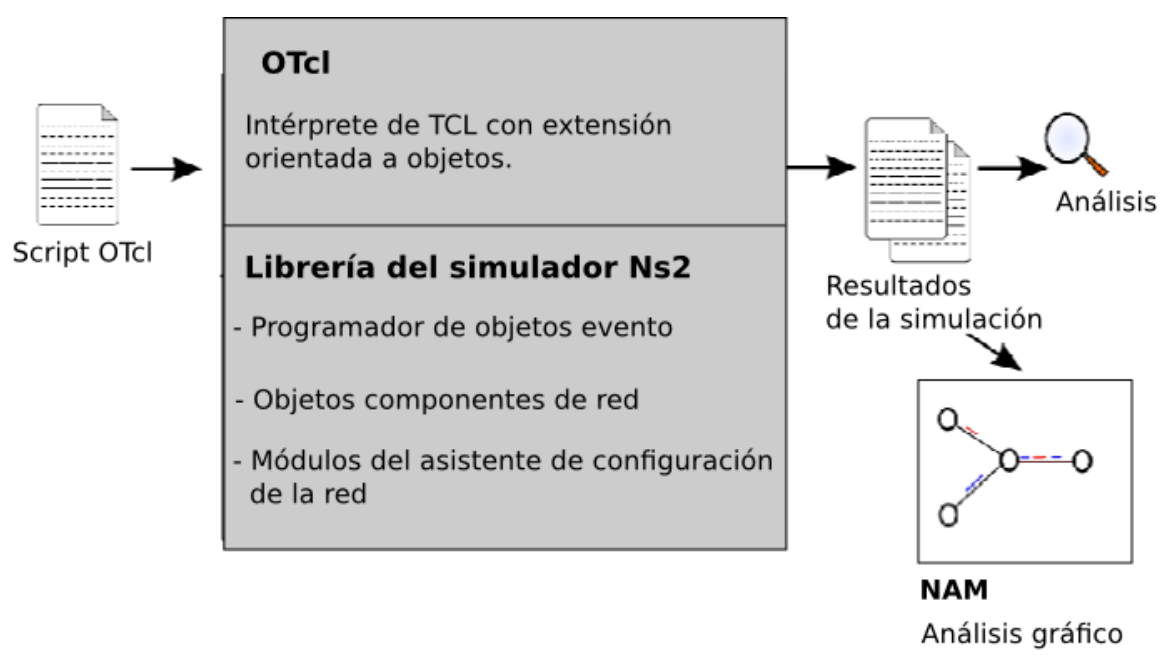

Figura A.2: Esquema simplificado de los elementos de NS-2.

permiten implementar el estándar IEEE 802.11 en NS-2, está fuera del alcance de esta sección. Se sugiere al lector interesado consultar [35] como un texto introductorio a los detalles de la implementación.

El modelo inalámbrico para NS-2 consta del objeto MobileNode como núcleo, con soporte de características adicionales de apoyo que permite la simulación de redes multi-hop, adhoc, WLAN, etc. El modelo fue desarrollado originalmente por el grupo Monarch de la CMU (Universidad Carnegie Mellon) como una extensión al simulador para el estudio de la movilidad en escenarios de simulación inalámbrica. La clase $\mathrm{C}++$ MobileNode se deriva de la clase padre Node. Un objeto MobileNode es básicamente un objeto Node con características inalámbricas de movilidad como el desplazarse dentro de una topología de red, la habilidad de transmitir y recibir mensajes desde y hacia el canal inalámbrico, etc. La principal diferencia entre estas clases radica en que los objetos de la clase MobileNode no están conectados por medio de la clase Link con otros objetos de su clase o de la clase Node.

La clase MobileNode es una clase compuesta, los componentes de la clase son las clases Wireless Channel, Network-Interface, Radio Propagation Model, MAC Protocols, Interface Queue, Link Layer and Address Resolution Protocol Model (ARP). Cuando se invoca una instancia a un objeto MobileNode éste existe como la interacción entre los objetos de las clases componentes como instancias separadas entre sí o como una instancia conjunta entre todos los objetos MobileNode presentes en la simulación, (tal es el caso de la clase Wireless Channel). Un diagrama de la estructura de jerarquías de la clase MobileNode se muestra en la Figura A.3 [4].

\section{A.1.2. IEEE 802.11e en NS-2}

El objetivo de la presente sección es describir las modificaciones en la estructura del simulador que permiten implementar el mecanismo de acceso EDCF/HCF sobre IEEE 802.11 en NS-2. Estas nuevas funciones de control de acceso al medio a nivel de la capa MAC han sido introducidas por IEEE en el estándar 802.11e [1] con el objetivo de ofrecer soporte de 


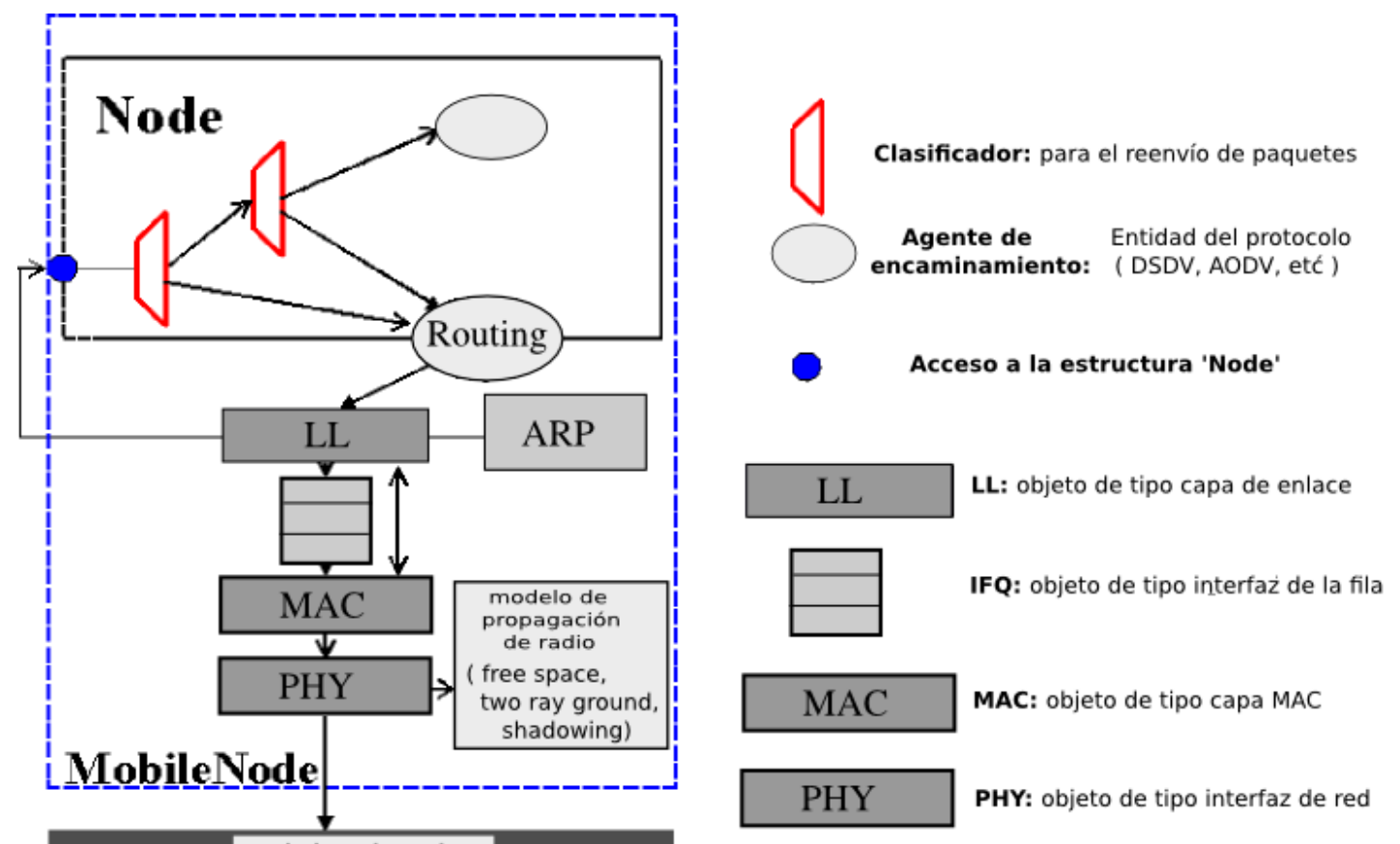

Figura A.3: Estructura de jerarquías para la clase MobileNode en NS-2.

calidad de servicio para las redes WLAN existentes. En este apartado no se pretende hacer una descripción exhaustiva de la implementación, se sugiere el lector interesado en conocer a detalle las modificaciones al código de NS-2 consultar [5].

La Figura A.4 muestra los módulos añadidos a la estructura de jerarquías para la clase MobileNode. Las nuevas instancias de clase Mac802_11HC y Mac802_11eHC implementan las funciones de acceso al medio EDCA y HCCA, respectivamente. Ambas clases heredan sus atributos de la clase MAC para MobileNode y extienden su funcionalidad para conseguir la modularidad deseada en el acceso al medio propuesta para el estándar.

La implementación del mecanismo de acceso EDCA hace uso de la clase MAC propuesta originalmente para la estructura MobileNode (la clase Mac802_11) con el fin de efectuar algunas operaciones comunes para cada categoría de tráfico $(\mathrm{AC})$, mientras que la nueva clase Mac802_11HC emula el algoritmo de acceso para cada una de las filas de espera con su propio conjunto característico de parámetros EDCA.

Debido a que cada AC necesita su propia fila de espera, una diferencia importante entre la estructura original para la clase MobileNode y la que se plantea en la propuesta [5] es la implementación simultánea de 4 filas de espera (una por cada AC). Aunque la extensión al simulador considera únicamente filas de espera para $2 \mathrm{AC}$ (tráfico best-effort y tráfico multimedia), para los fines del proyecto se implementan filas de espera para cada una de las $4 \mathrm{AC}$, (como es requerido por el estándar [1]).

La extensión hace un uso extensivo de los métodos de la clase Mac-timers para gestionar la diferenciación del tráfico por categoría, coordinar los intervalos de tiempo para el intercambio de tramas y establecer la duración de los periodos de acceso al canal con contención (CAP) y libre de contención (CFP) mediante la planificación del envío de tramas de señalización. 


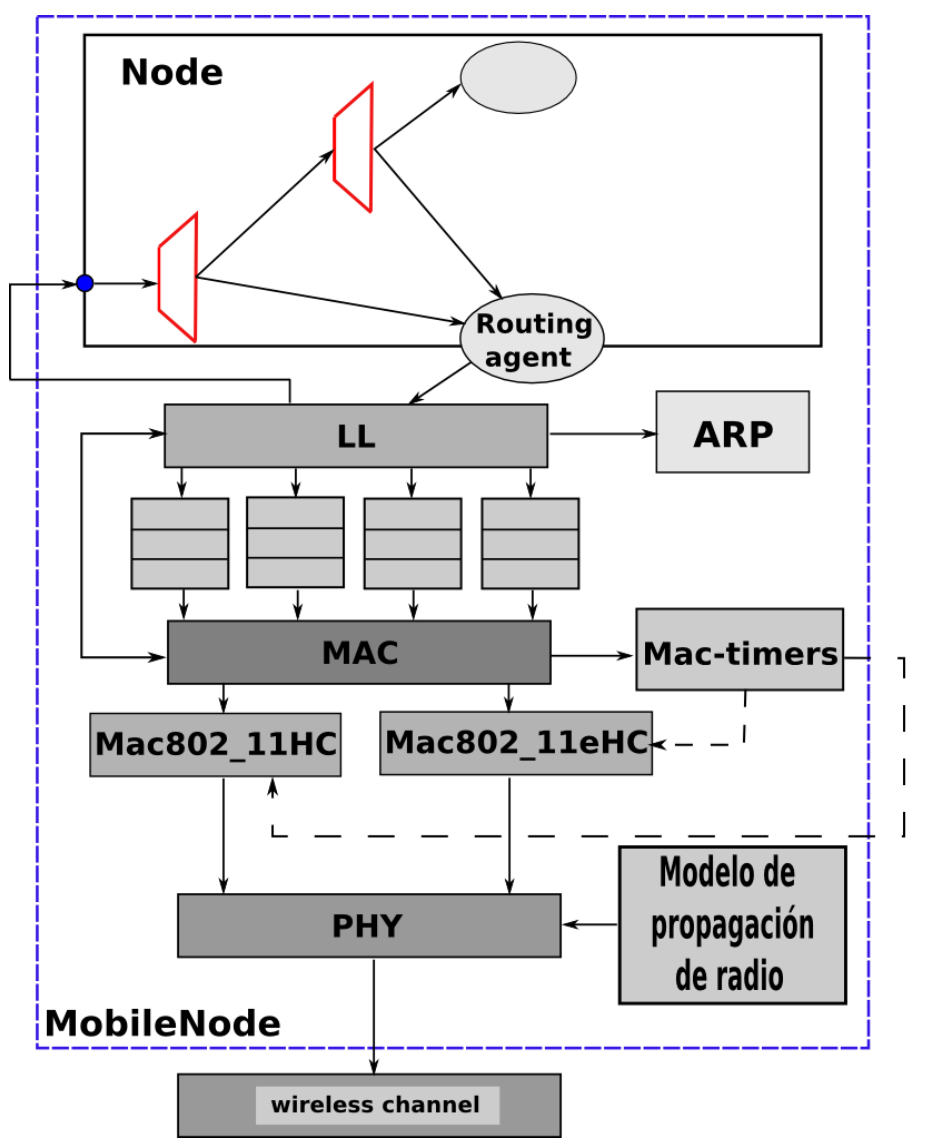

1

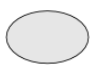

0

LL

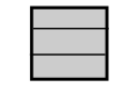

MAC

Mac802_11HC

Mac802_11eHC

Macs

PHY
Clasificador: para el reenvío de paquetes

Agente de Entidad del protocolo encaminamiento: (DSDV, AODV, etć )

Acceso a la estructura 'Node'

LL: objeto de tipo capa de enlace

IFQ: objeto de tipo interfaz de la fila

MAC: objeto de tipo capa MAC

Mac802_11HC: objeto heredado de la capa MAC (implementa el mecanismo de EDCA)

eHC: objeto heredado de la capa MAC (implementa el mecanismo de HCCA)

PHY: objeto de tipo interfaz de red

Figura A.4: Estructura de jerarquías propuesta en [5] para la clase MobileNode de NS-2.

Considerando otras extensiones populares para el estudio del estándar IEEE 802.11e para NS-2 ${ }^{1}$. Una característica destacada en esta implementación es que permite configurar el simulador para realizar simulaciones usando EDCA, HCCA o EDCA+HCCA como función de acceso al medio a nivel de la capa MAC. Esto nos resulta especialmente útil al realizar la evaluación de desempeño de los algoritmos EDCA modificados que se presenta en la siguiente sección, mientras se respeta la integridad del estándar. La implementación parcial del estándar [1] en varias de las extensiones disponibles al código del simulador puede conducir a resultados cuestionables sobre la validez en la evaluación de desempeño mediante simulación con NS-2.

\footnotetext{
${ }^{1}$ La dirección: http : //nsnam.isi.edu/nsnam/index.php/Contributed_Code. Constituye un excelente punto de partida para obtener información sobre otras extensiones disponibles al código de NS- 2 .
} 


\section{Acrónimos}

\section{A}

AC (Categoría de Acceso)

Valor que indica la prioridad del tráfico cuando se utiliza el mecanismo EDCA. Existe una fila de espera por cada categoría de acceso.

\section{ACK (Trama de Confirmación)}

En las redes de computadoras, un acuse de recibo (o reconocimiento) es una trama de confirmación que es pasada entre las estaciones para indicar el reconocimiento o la correcta recepción de la respuesta en el destino.

\section{AIFS (Espacio entre Tramas Arbitrario)}

En el modo EDCA, tras una ranura de tiempo ocupada, es el tiempo que debe esperar una estación hasta poder decrementar su contador de backoff y que depende de la categoría de acceso en cuestión.

\section{AP (Punto de Acceso)}

Dispositivo que realiza la función de proporcionar a las estaciones dentro de la WLAN conectividad con el exterior de la red (generalmente el acceso a Internet).

\section{B}

\section{Backoff (Algoritmo de Espera Aleatoria)}

Algoritmo según el cual se determina una espera adicional y aleatoria escogida uniformemente en un intervalo llamado ventana de contienda. El algoritmo de backoff nos da un número aleatorio y entero de ranuras de tiempo y su función es la de reducir la probabilidad de colisión que es máxima cuando varias estaciones están esperando a que el medio quede libre para transmitir.

\section{BS (Tamaño de la Ráfaga)}

Parámetro de la especificación del tráfico que nos indica el máximo tamaño de ráfagas de datos de un determinado flujo de datos.

\section{BSS (Conjunto Básico de Servicio)}

Son los servicios que soportan la comunicación entre estaciones.

\section{BSA (Área de Servicio Básico)}

Área dentro de la cual una estación puede comunicarse con otras estaciones, que también pertenecen al área de servicio básico. 
C

CAP (Período de Acceso Controlado)

Un período de acceso controlado consiste en una o más TXOPs, durante las cuales el QAP puede transmitir SDUs que pertenecen a un flujo descendente o enviar un mensaje de polling a una o más estaciones, especificando el tiempo máximo durante el que pueden ocupar el medio. Todo esto dentro de un intervalo de contención.

CBR (Tasa de Bit Constante)

Tráfico de usuario caracterizado por una velocidad de transmisión constante.

CDF (Función de Densidad de Probabilidad Acumulada)

Nos indica la probabilidad de que la variable aleatoria sea menor a un valor $x$.

CFP (Período Libre de Contención)

Durante el acceso al canal en IEEE 802.11, es el período en el que el coordinador puntual controla quién transmitirá, eliminando la necesidad de que las estaciones compitan por conseguir el acceso al canal.

\section{CP (Período de Contención)}

Durante el acceso al canal en IEEE 802.11, período en el cual las estaciones compiten por el uso del medio de transmisión ( i.e., el canal inalámbrico). nes)

CSMA/CA (Acceso Múltiple con Detección de Portadora Evitando ColisioProtocolo de control de acceso al medio que permite que varias estaciones utilicen un mismo medio de transmisión. CSMA/CA no detecta colisiones, pero sí intenta minimizar su ocurrencia.

CSMA/CD (Acceso Múltiple por Detección de Portadora con Detección de Colisiones)

Protocolo de control de acceso al medio en el que cada estación que desea transmitir, previamente escucha el canal, detectando posibles colisiones.

\section{CTS (Clear To Send)}

En el modo RTS/CTS, este mensaje indica a una estación que puede transmitir.

\section{CW (Ventana de Contención)}

Parámetro del estándar IEEE 802.11 que corresponde al valor máximo posible para el período aleatorio de backoff.

\section{$\mathrm{D}$}

\section{D (Retardo)}

Variable de la especificación de tráfico que define el tiempo máximo para el transporte de tramas. El retardo incluye la propagación por el medio inalámbrico, el periodo de contención en el acceso al canal y el tiempo de espera en la fila.

\section{DCF (Función de Coordinación Distribuida)}

Función de la capa MAC que permite la transmisión de datos de acuerdo con el método de mejor esfuerzo. Con DCF, todas las estaciones compiten por conseguir el acceso al canal cada vez que intentan transmitir. 


\section{DIFS (Espacio entre Tramas DCF)}

Tiempo mínimo que el medio ha de estar desocupado antes de iniciar la transmisión durante el período de contención.

\section{DSSS (Espectro Extendido por Secuencia Directa)}

Método de modulación en espectro extendido para transmisión de señales digitales. Esta técnica utiliza un código pseudoaleatorio para modular la señal a transmitir.

\section{$\mathrm{E}$}

\section{EDCA (Acceso Coordinado y Distribuido Mejorado)}

Función de acceso al canal de IEEE 802.11e basada en contención, y que constituye una ampliación de la función DCF. EDCA proporciona servicios de trafico diferenciado, sin reserva de recursos. En EDCA la decisión para asignar la TXOP se toma conjuntamente entre todas las estaciones.

\section{EDCA-CI (Algoritmo EDCA orientado a clasificación inter-estación)}

Mecanismo de acceso compatible con el modo EDCA del estándar IEEE 802.11e, en el que la asignación de la TXOP se obtiene en base a un esquema de prioridades ínter-estación basado en conteo de tráfico local.

\section{EIFS (Espacio Entre Tramas Extendido)}

Tiempo entre tramas usado para retransmisiones, siendo el espacio entre trama de mayor duración.

\section{ESS (Conjunto de Servicios Extendidos)}

Conjunto de servicios que permiten la unión de varias BSSs mediante un sistema de distribución.

\section{$\mathbf{F}$}

FDDI (Interfaz de Datos Distribuidos por Fibra)

Estándar para la transmisión de datos en redes de computadoras de área extendida o de área local mediante cable de fibra óptica.

\section{FHSS (Espectro Extendido por Salto de Frecuencia)}

Es una técnica de modulación en espectro extendido en el que la señal se emite sobre una serie de frecuencias pseudoaleatorias, saltando de frecuencia en frecuencia de forma síncrona con el receptor.

\section{FTP (Protocolo de Transferencia de archivos)}

Es un protocolo de red para la transferencia de archivos entres sistemas conectados a una red TCP, basado en la arquitectura cliente-servidor.

\section{$\mathbf{H}$}

HCCA (Mecanismo de Acceso al Medio Controlado por HCF)

Es una ampliación de PCF para proveer QoS. Se puede usar tanto en el intervalo de contención como en el intervalo libre de contención. Siendo una función centralizada, el acceso al canal está basado en el envío de mensajes de polling. 


\section{HCF (Función de Coordinación Híbrida)}

Es la función encargada de controlar el acceso al medio en el estándar IEEE802.11e.

\section{HC (Coordinador Híbrido)}

En HCCA, es un dispositivo encargado de decidir a qué estación le toca transmitir. Normalmente coincide con el QAP.

\section{I}

\section{IBSS (Conjunto de Servicio Básico Independiente)}

Conjunto de servicios que soportan la comunicación entre estaciones, sin usar un punto de acceso.

\section{IP (Protocolo de Internet)}

Protocolo que corresponde con la capa de red de la arquitectura TCP/IP, y proporciona a la capa de transporte un servicio no orientado a conexión y de entrega de mejor esfuerzo.

IR (Infrarrojo)

Radiación electromagnética con longitudes de onda entre 700 nanómetros hasta 1 milímetro.

ISM (Industrial, Científico y Médicas)

Son bandas reservadas internacionalmente para un uso no comercial radiofrecuencias electromagnéticas en las áreas industrial, científica y médica.

\section{L}

\section{LAN (Red de Área Local)}

Conjunto de estaciones y dispositivos de red que están interconectados mediante un medio de transmisión compartido.

\section{LLC (Control del Enlace Lógico)}

Subcapa de la capa de enlace que se encarga del control de errores, el control de flujo y el manejo de las tramas.

\section{$\mathrm{M}$}

M (Tamaño Máximo de la SDU)

Parámetro de la especificación del tráfico que define el tamaño máximo de la SDU.

\section{MAC (Control de Acceso al Medio)}

Subcapa de la capa de enlace que se encarga de coordinar el acceso al canal de forma que la información vaya desde el origen al destino a través de la misma red de difusión.

MACAW (Control de Acceso al Medio con Prevención de Colisiones para Redes Inalámbricas)

Algoritmo de acceso al canal para el modo RTS/CTS.

\section{MPDU (Unidad de Datos del Protocolo MAC)}

Son los mensajes (unidad de datos del protocolo) intercambiados entre las entidades MAC dentro del BSS. 
MSDU Unidad de datos de servicio MAC)

Unidad de datos recibida en la subcapa de control de acceso al medio (MAC) proveniente de la subcapa de enlace lógico (LLC).

\section{Msi (Mínimo Intervalo de Servicio)}

Parámetro del TSPEC que indica el intervalo de tiempo mínimo entre el comienzo de períodos de servicio sucesivos.

\section{MSI (Máximo Intervalo de Servicio)}

Parámetro del TSPEC que indica el intervalo de tiempo máximo entre el comienzo de períodos de servicio sucesivos.

\section{$\mathrm{N}$}

N (Tamaño Nominal de la SDU)

Variable del TSPEC que define el tamaño promedio de la SDU.

\section{NAV (Vector de Asignación de red)}

Este vector actualiza el contador interno de la estación para que no transmita durante un periodo de tiempo específico y de esta forma poder realizar transmisiones procedentes de otras estaciones en este periodo.

\section{NS2 (Network Simulator 2)}

Simulador de redes de eventos discretos y orientado a objetos desarrollado por la Universidad de Berkeley en California y escrito en $\mathrm{C}++$ y OTcl.

\section{$\mathrm{O}$}

OFDM (Multiplexación por División de Frecuencias Ortogonales)

Es una técnica de modulación que consiste en enviar un conjunto de portadoras de diferentes frecuencias donde cada una transporta información modulada mediante una técnica distinta.

\section{$\mathbf{P}$}

PC (Coordinador Puntual)

En la función PCF, el coordinador puntual es el encargado de decidir qué estación tiene el turno para poder transmitir.

\section{PCF (Función de Coordinación Puntual)}

Función centralizada de la capa MAC. Utiliza un coordinador puntual para repartir a las estaciones los turnos de transmisión.

\section{PIFS (Espacio Entre Tramas PCF)}

Tiempo de espera antes de poder transmitir una trama durante el período libre contención. Como el intervalo PIFS es menor que el período DIFS, estas transmisiones tendrán prioridad durante dicho período.

PMD (Capa Dependiente del Medio Físico)

Subcapa encargada de la modulación y codificación dentro de la capa física. 
PLCP (Procedimiento de Convergencia de la Capa Física)

Subcapa de la capa física encargada de tratar las tramas generadas en la subcapa MAC y adaptarlas al formato adecuado para la subcapa dependiente del medio físico.

Q

QAP (Punto de Acceso con QoS)

Punto de acceso con soporte de calidad de servicio.

QoS (Calidad de Servicio)

Parámetro multidimensional que caracteriza lo que demanda la aplicación a lo que ofrece la red.

QSTA (Estación con QoS)

Estación con soporte de calidad de servicio.

QBSS (Conjunto de Servicio Básico con QoS)

Conjunto de servicio básico con soporte de calidad de servicio.

$\mathbf{R}$

RAMPS (Algoritmo de asignación de prioridades intra-AC)

Algoritmo de asignación de prioridades intra-AC [2] para el mecanismo de acceso al medio EDCA del estándar IEEE 802.11e.

R (Tasa Mínima en la Capa Física)

Parámetro de la especificación del tráfico que define la tasa de bit mínima en el medio inalámbrico.

\section{RTS (Mensaje de Petición de Envío)}

En el modo RTS/CTS, este mensaje indica que una estación solicita un turno de transmisión.

$\mathrm{S}$

SDU (Unidad de Datos de Servicio)

Es la unidad de datos, dentro del sistema de referencia OSI que, dada una capa concreta, no incluye la información de control o cabecera de dicha capa.

SI (Intervalo de servicio)

Intervalos de tiempo durante el cual son asignadas las TXOP por EDCA o HCCA durante el tiempo de la super-trama IEEE 802.11e.

SIFS (Espacio entre tramas corto)

Tiempo de espera antes de realizar transmisiones de alta prioridad (e.g. tramas ACK). También se utiliza para transmisiones de las estaciones durante el período libre de contención. Debido a su corta duración, este tipo de transmisión no es proclive a presentar colisiones.

\section{SSID (Identificador de Conjunto de Servicios)}

Es un código incluido en todos los paquetes de una red inalámbrica para identificarla. El código consiste en un máximo de 32 caracteres alfanuméricos. Todos los dispositivos inalámbricos que intentan comunicarse entre sí tienen que compartir el mismo SSID. A menudo al SSID se le conoce como nombre de red. 
SST (Tiempo de Comienzo del Servicio)

Parámetro del TSPEC que nos indica el momento en el que el intervalo de servicio comienza.

\section{STA (Estación)}

Acrónimo asignado a los equipos terminales de usuario. También se le conoce como los nodos de red.

$\mathrm{T}$

TCP/IP (Protocolo de Control de Transmisión/Protocolo de Internet)

Es un conjunto de protocolos en el que se basa Internet y que permiten la transmisión de datos entre redes de computadoras. Hace referencia a los dos protocolos más importantes que lo componen: Protocolo de Control de Transmisión (TCP) y Protocolo de Internet (IP).

\section{TCP (Protocolo de Control de Transmisión)}

Protocolo perteneciente a la capa de transporte de la arquitectura TCP/IP. Proporciona un servicio con control de flujo, de congestión y de errores.

TGe (Grupo de Trabajo E)

Grupo encargado de diseñar el estándar IEEE 802.11e.

TID (Identificador de Tráfico)

Este identificador tomará un valor entre 0 y 15, según sus requisitos de QoS. Las tramas con valores TID entre 0 y 7 son mapeadas en cuatro colas, correspondientes a cuatro categorías de acceso (ACs) y usan reglas de acceso EDCA. Por el contrario, las tramas marcadas con un TID entre 8 y 15 son mapeadas en ocho tipos de flujo de tráfico (TS) y usan reglas HCCA.

TS (Flujo de Tráfico)

En IEEE 802.11e define un flujo de datos, ascendente y descendente, con calidad de servicio.

TSID (Identificación del Tipo de flujo)

Parámetro del TSPEC para identificar cada uno de los flujos de una estación.

\section{TSPEC (Especificación del Tráfico)}

Conjunto de parámetros que definen las características del tráfico de una determinada estación.

TBTT (Tiempo de Transmisión de la Trama de Señalización)

Parámetro que indica el tiempo entre transmisión de dos tramas de señalización.

\section{Throughput (Tasa de Bit)}

La tasa de bits es la relación de bits por segundo que consume un archivo de datos, audio o de vídeo.

Time-Out (Intervalo de Espera Antes de Solicitar una Retransmisión)

Es un intervalo de tiempo durante el cual una QSTA espera el acuse de recibo de la trama enviada. Si el ACK no se recibe dentro del límite de tiempo (condición de Time-Out), se asumirá que ocurrió una colisión y se retransmitirá la trama. 
TXOP (Oportunidad de Transmisión)

Es un intervalo de tiempo limitado durante el cual una QSTA puede transmitir una serie de tramas.

$\mathbf{U}$

UDP (Protocolo de Datagramas de Usuario)

Es un protocolo de nivel de transporte que permite el envío de información a través de la red sin que se haya establecido previamente una conexión, existan confirmaciones ó control de flujo y/o congestión.

UN-II (Infraestructura de Información Nacional sin Licencia)

Banda del espectro radiofrecuencia entre 5,15-5,825GHz.

\section{$\mathrm{V}$}

VC (Videoconferencia)

Tipo de aplicación de vídeo bajo demanda, en la que dos o varias personas situadas de forma remota, se comunican mediante vídeo y audio.

VoIP (Voz sobre IP)

Tecnología que hace posible que la señal de voz viaje a través de redes de datos empleando el protocolo IP (Protocolo Internet). Esto significa que se envía la señal de voz en forma digital en paquetes en lugar de enviarla (en forma digital o analógica) a través de circuitos utilizables sólo para telefonía.

\section{VoD (Video Bajo Demanda)}

Es un protocolo que permite a los usuarios acceder a contenidos multimedia de forma personalizada.

\section{W}

WLAN (Redes de Área Local Inalámbrica)

Redes de área local no cableadas. Existen varios tipos de tecnologías WLAN, como pueden ser IEEE 802.11 o HiperLAN.

\section{WDS (Sistema de Distribución Inalámbrica)}

Un sistema de distribución inalámbrico (WDS) es un sistema que permite la interconexión inalámbrica de puntos de acceso en una red IEEE 802.11. Permite que una red inalámbrica pueda ser ampliada mediante múltiples puntos de acceso sin la necesidad de un cable troncal (medio físico) que los conecte. 